\title{
The Importance of Mindfulness and Self-Compassion in Clinical Training: Outcomes Related to Self-Assessed Competency and Self-Efficacy in Psychologists-in-Training
}

\author{
Chelsea Latorre \\ chlatorre@mix.wvu.edu
}

Follow this and additional works at: https://researchrepository.wvu.edu/etd

Part of the Clinical Psychology Commons, Counseling Commons, and the Counseling Psychology Commons

\section{Recommended Citation \\ Latorre, Chelsea, "The Importance of Mindfulness and Self-Compassion in Clinical Training: Outcomes Related to Self-Assessed Competency and Self-Efficacy in Psychologists-in-Training" (2020). Graduate Theses, Dissertations, and Problem Reports. 7792. \\ https://researchrepository.wvu.edu/etd/7792}

This Dissertation is protected by copyright and/or related rights. It has been brought to you by the The Research Repository @ WVU with permission from the rights-holder(s). You are free to use this Dissertation in any way that is permitted by the copyright and related rights legislation that applies to your use. For other uses you must obtain permission from the rights-holder(s) directly, unless additional rights are indicated by a Creative Commons license in the record and/ or on the work itself. This Dissertation has been accepted for inclusion in WVU Graduate Theses, Dissertations, and Problem Reports collection by an authorized administrator of The Research Repository @ WVU. For more information, please contact researchrepository@mail.wvu.edu. 
The Importance of Mindfulness and Self-Compassion in Clinical Training: Outcomes Related to Self-Assessed Competency and Self-Efficacy in Psychologists-in-Training

Chelsea Latorre 
The Importance of Mindfulness and Self-Compassion in Clinical Training:

Outcomes Related to Self-Assessed Competency and Self-Efficacy in Psychologists-inTraining

Chelsea Latorre, M.Ed.

Dissertation submitted to the

College of Education and Human Services

at West Virginia University

in partial fulfillment of the requirements for the degree of

Doctor of Philosophy in

Counseling Psychology

Monica Leppma, Ph.D., Chair

Jeff Daniels, Ph.D.

Lisa F. Platt, Ph.D.

Natalie Shook, Ph.D.

Department of Counseling, Rehabilitation Counseling, and Counseling Psychology

Morgantown, West Virginia

2019

Keywords: mindfulness; self-compassion; training; counseling; self-efficacy; competency

Copyright 2019 Chelsea A. Latorre 


\begin{abstract}
The Importance of Mindfulness and Self-Compassion in Clinical Training:

Outcomes Related to Self-Assessed Competency and Self-Efficacy in

Psychologists-in-Training
\end{abstract}

Chelsea Latorre, M.Ed.

The release of the APA (2011) Revised Competency Benchmarks used for evaluation of doctoral-level psychology trainees calls for literature to examine competencies in this population with use of appropriate instrumentation. The recent publication and validation of the Professional Competencies Scale-Revised (Taylor, 2015) allows for researchers to explore factors associated with trainee competencies as it relates to the benchmark system. Previous literature suggests a strong relationship between counselor self-efficacy and professional competency (e.g., Constantine, 2002), suggesting that interventions that can foster these constructs may lead to enhanced counseling performance. This study examined the predictive value of mindfulness and self-compassion for self-assessed professional competency and counselor self-efficacy in a sample of counseling and clinical psychology doctoral trainees $(n=192)$. The relationships among mindfulness, self-compassion and training year were also explored. The results of stepwise linear regression analyses revealed that higher levels of mindfulness were associated with greater levels of self-compassion, and higher levels of self-compassion were associated with greater levels of counselor self-efficacy and self-assessed professional competency. Findings also indicated potential mediating effects that self-compassion has on the relationship between mindfulness and outcomes variables (counselor self-efficacy and self-assessed professional competency). This study provides relevant information to operationalize self-compassion and mindfulness as contrasting, yet similar, constructs. In addition, similarities and differences between counselor self-efficacy and perceived competency are highlighted. Implications for 
educational programs and training sites are discussed, as well as strengths and limitations of the study and suggestions for future research. 


\section{DEDICATION}

This dissertation is dedicated to striving and thriving counseling and clinical psychologists, to devoted faculty and supervisors in fields of psychology, and to the future of health service psychology. 


\section{ACKNOWLEDGEMENTS}

First and foremost, thank you to my support system that has always held strong in celebrating my successes and achievements and helping me through any and all adversities. I'm thankful for my family and their unconditional love and support throughout every step of my career journey. I have appreciated being a part of such an amazingly devoted and cohesive cohort, and I thank each one of you for being yourselves and standing alongside me every step of the way. In general, thanks to the friends I have made in this program that have helped me thrive. I would not have challenged myself, personally and professionally, as much as I have (and will continue to do) without this support and encouragement.

Moving across the country for my career could have been a difficult task if it wasn't for the fantastic group of people I have surrounded myself with. I want to acknowledge and thank the friends that I have made all over the country for sharing experiences of a lifetime and for your constant support. A special thanks to Seger for the many miles and years you have devoted to me and my career. Thank you to my family and peers for always believing in me. The achievement of completing this dissertation is a result of my meaningful relationships with you all - thanks for encouraging me to be my best.

I would also like to acknowledge and thank the members of my committee, Dr. Monica Leppma, Dr. Jeff Daniels, Dr. Lisa Platt, and Dr. Natalie Shook. I have learned so much from each of you and I deeply appreciate your guidance and support throughout this dissertation process. In addition, I am grateful to have had several wonderful supervisors at the Carruth Center at West Virginia University who have shared wisdom and helped to develop my identity as a professional psychologist. As faculty and supervisors, you all have encouraged me to find my voice and, through this process, I have developed a passion for training and education. 
Lastly, I want to acknowledge and thank the College of Education and Human Services at West Virginia University. The college had awarded me a research grant to help fund aspects of data collection to complete this dissertation. Additionally, the college has awarded me two general scholarships that have helped to fund my education. These resources have been extremely helpful in my educational career. 


\section{TABLE OF CONTENTS}

ABSTRACT .ii

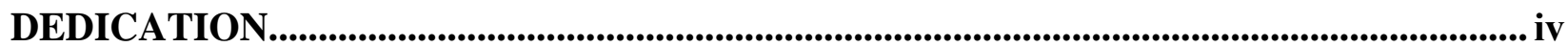

ACKNOWLEDGEMENTS .................................................................................................

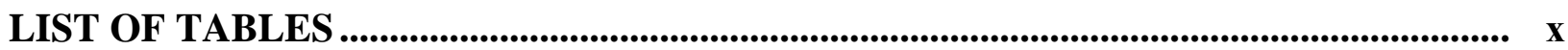

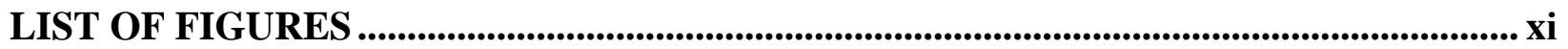

CHAPTER 1: INTRODUCTION AND LITERATURE REVIEW ................................... 1

Professional Competency in Training Programs ................................................................. 4

Professional Competencies Benchmark Standards ....................................................... 6

Self-Assessment of Professional Competency ................................................................ 7

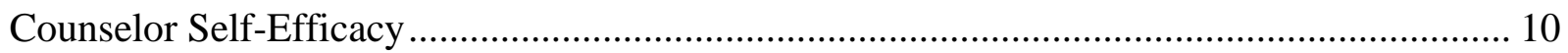

Self-Assessed Competency and Counselor Self-Efficacy: Contrasting Constructs ................ 12

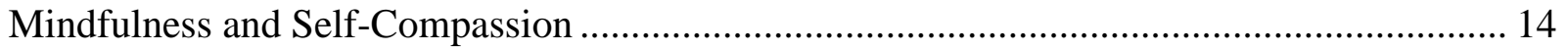

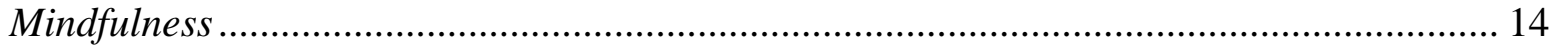

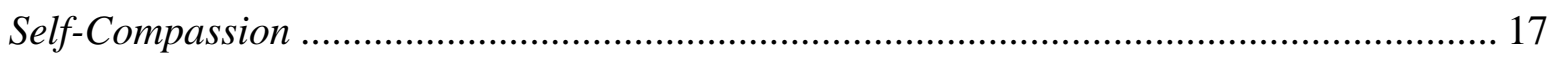

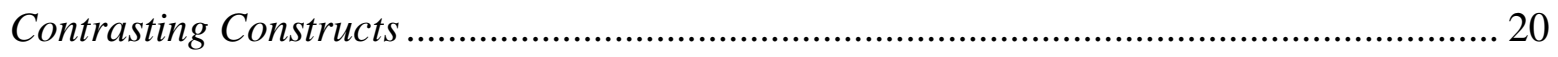

Mindfulness and Self-Compassion in Training .......................................................... 22

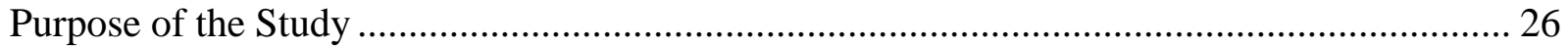

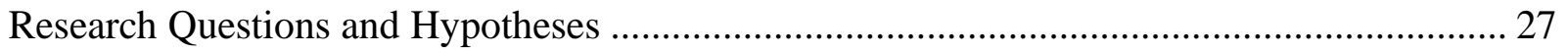

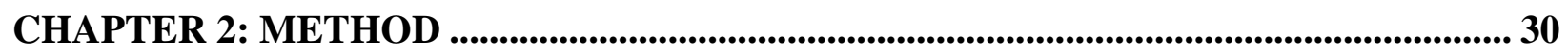

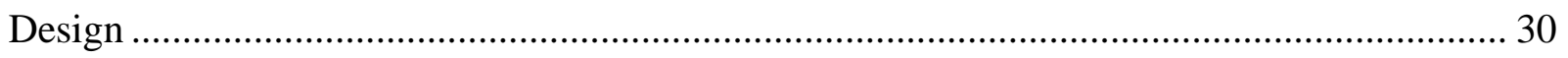

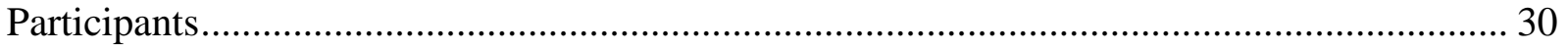

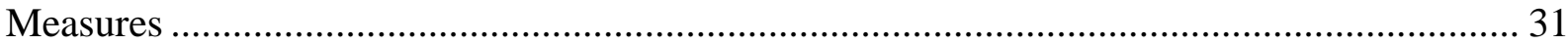

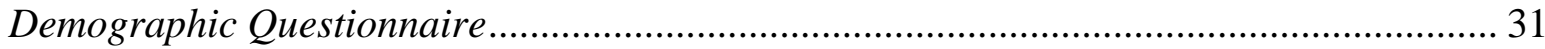

Mindful Attention Awareness Scale (MAAS; Brown \& Ryan, 2003a) ................................ 32

Self-Compassion Scale Short-Form (SCS-SF; Raes et al., 2011) ..................................... 34

Counselor Self-Efficacy Scale (CSES; Melchert et al., 1996) ......................................... 35

Professional Competencies Scale-Revised (PCS-R; Taylor, 2015) .................................. 36

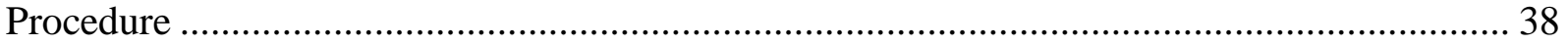

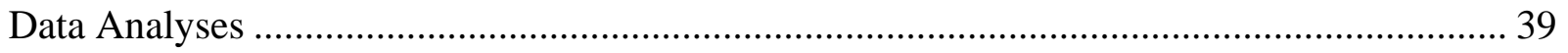

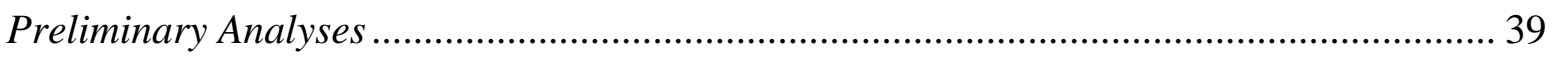

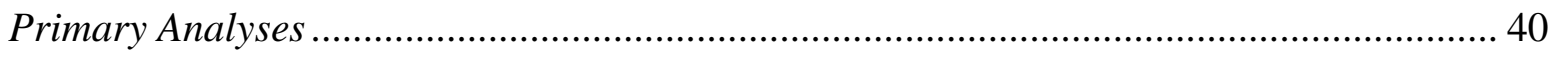

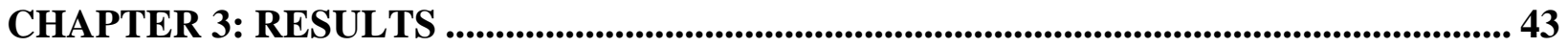




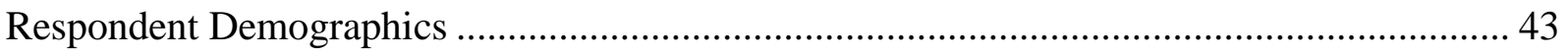

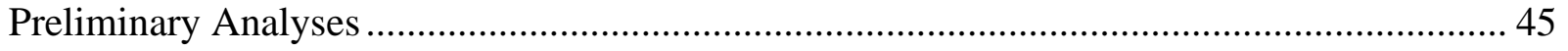

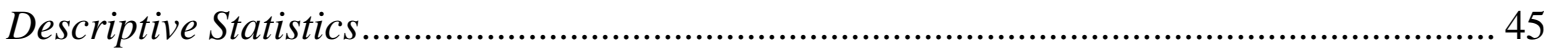

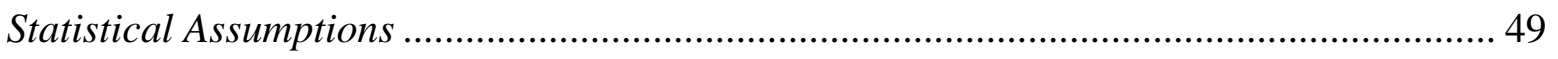

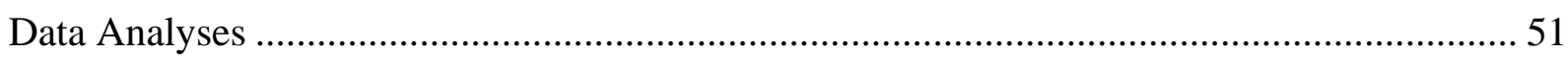

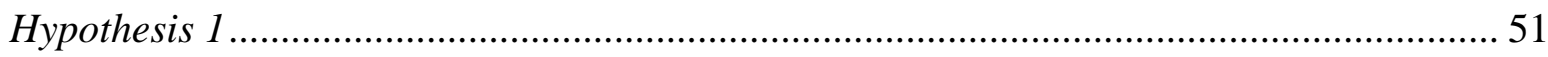

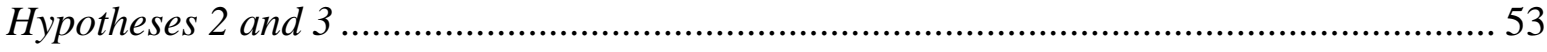

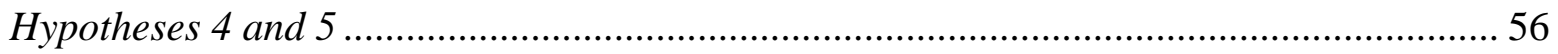

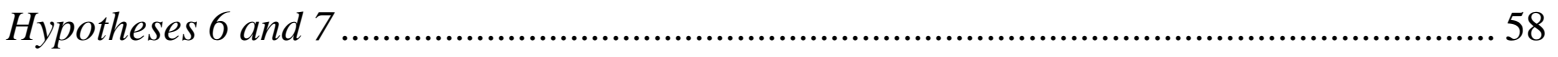

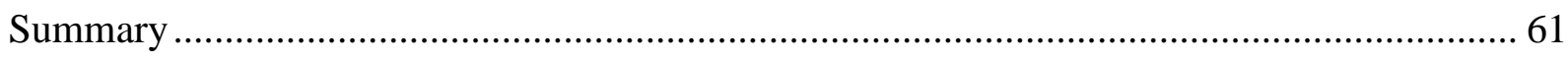

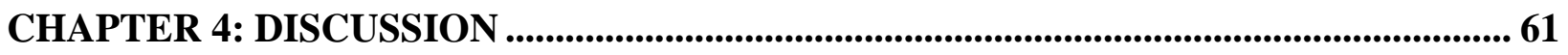

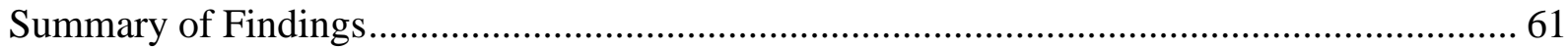

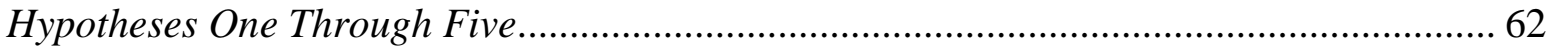

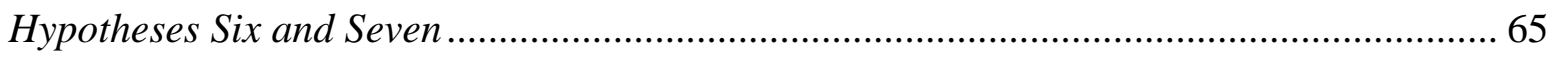

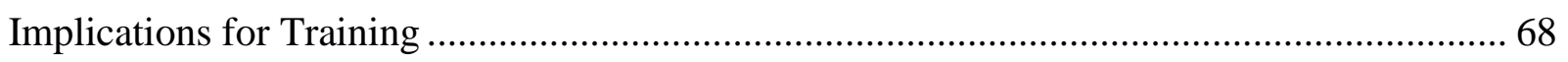

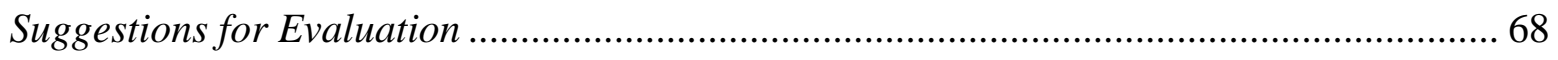

Informal Mindfulness and Self-Compassion Practices ................................................. 70

Formal Mindfulness and Self-Compassion Practices ................................................... 72

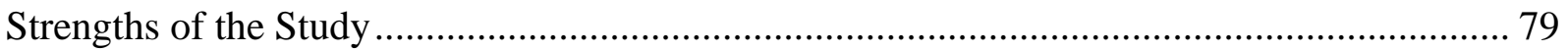

Limitations and Recommendations for Future Research.............................................. 81

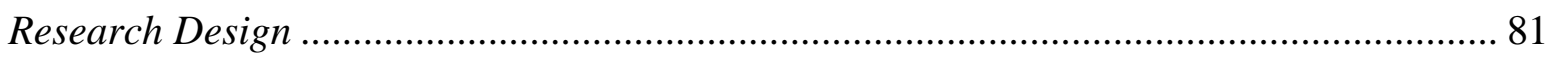

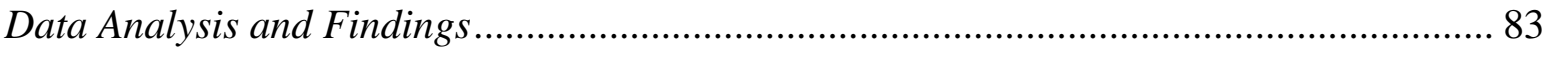

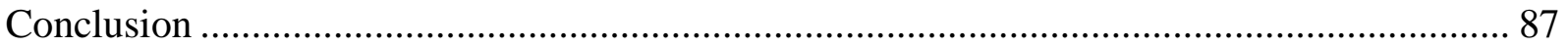

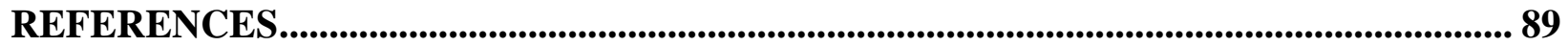

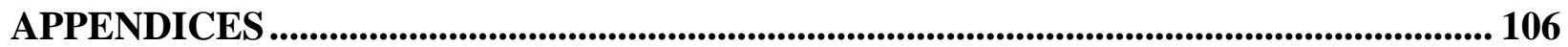

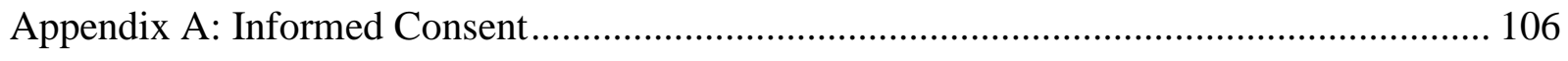

Appendix B: Participant Invitation Request ......................................................... 108

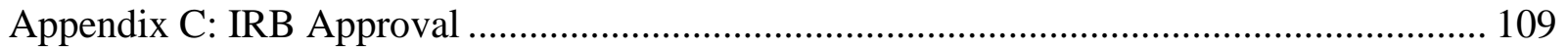

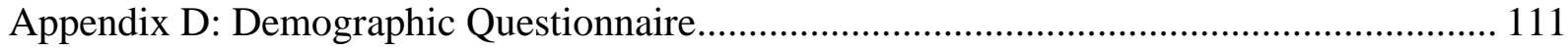

Appendix E: Mindful Attention Awareness Scale (MAAS) ......................................... 113

Appendix F: Self-Compassion Scale Short-Form (SCS-SF) .......................................... 114

Appendix G: Counselor Self-Efficacy Scale (CSES) …................................................ 115 
Appendix H: Professional Competencies Scale- Revised (PCS-R) .................................. 116

Appendix I: Figures Related to Statistical Assumptions ............................................... 117 


\section{LIST OF TABLES}

Table 1: Participant Demographics.......................................................................................... 45

Table 2: Correlations Among Variables ................................................................................... 46

Table 3: Results of $t$-tests and Descriptive Statistics by Gender .................................................. 47

Table 4: Results of $t$-tests and Descriptive Statistics by Type of Program..................................... 48

Table 5: Results of $t$-tests and Descriptive Statistics by Degree .................................................. 48

Table 6: Results of $t$-tests and Descriptive Statistics by Race/Ethnicity ..................................... 49

Table 7: SLR Predicting SCS-SF from MAAS …………………………………………..... 53

Table 8: SLR Predicting CSES from MAAS and SCS-SF ........................................................... 55

Table 9: SCS-SF as a Mediating Variable between MAAS and CSES ........................................ 55

Table 10: SLR Predicting PCS-R from MAAS and SCS-SF ....................................................... 57

Table 11: SCS-SF as a Mediating Variable between MAAS and PCS-R ....................................... 57

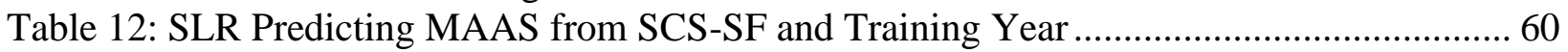

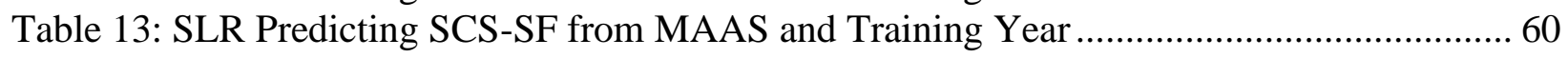




\section{LIST OF FIGURES}

Figure 1. Competency Cube Model (Rodolfa et al., 2005)..................................................... 5

Figure 2. SCS-SF as a Mediator Variable between MAAS and CSES. .................................. 56

Figure 3. SCS-SF as a Mediator Variable between MAAS and PCS-R .................................. 58

Figure 4. Mean Scores of MAAS Across Training Year.......................................................... 67

Figure 5. Mean Scores of SCS-SF Across Training Year. .................................................... 68 


\section{CHAPTER 1: INTRODUCTION AND LITERATURE REVIEW}

The field of professional psychology promotes a culture of competence as one of its core values and initiatives for the future of education and training. The American Psychological Association (APA), the governing body of professional psychology, highlights the importance of standards of competence as it relates to ethics, achievement, conduct, and education in their mission statement (APA, 2018). Thus, counseling, clinical, and school psychology graduate programs across the nation are cultivating a culture of competence. Programs are expected to assess the competency of their students using a set of benchmark standards throughout the longevity of their program to determine the appropriateness of each student moving into practice. Faculty and supervisors use the benchmark standards to assess a student's developmental level and competence. These standards cover various areas of training, specifically the knowledge, skills, and attitudes that are necessary to excel their training program and throughout their career in practice.

Though the APA developed these standards of evaluation to comprehensively assess students' development and competence across various domains, the evaluation document is missing a key factor involved in assessing competence. The benchmark standards are set in place for faculty and supervisors to assess their trainee's knowledge, skills, and abilities, but this evaluation process omits trainees' active self-appraisal. Moreover, the guidebook associated with the benchmark standards highlights that students should be oriented and introduced to the evaluation system set forth by the APA and their respective training programs (APA, 2012). However, students are only passively involved in the evaluation process by receiving their supervisors' feedback using this system. Though trainees are often expected to be engaged in self-evaluation and self-awareness practices throughout their training and in their practicum 
experiences (Ridley et al., 2011), their involvement in this practice is overlooked in the formal evaluation process using the benchmark standards.

Supervisors often examine the effects of psychology trainees' self-awareness while they are working with clients in a therapeutic setting (Fauth \& Williams, 2005). However, beyond therapeutic effects and outcomes, self-awareness is also important within the context of competency. Ridley et al. (2011) argue that self-evaluation allows individuals to "examine their performance, recognize their strengths and weaknesses, understand their boundaries of competence, and continually assess their work" (p. 21). Given that trainees are a major part of the evaluation process, coupled with the significance of self-appraisal and self-evaluation demonstrated throughout the literature (e.g., Belar et al., 2001; Hughes et al., 2011; Roberts et al., 2005), I argue that an important part of trainee development and evaluation should include trainees' self-appraisal. Thus, the present study will provide an opportunity for trainees to selfassess their perceived competency and self-efficacy in relation to other aspects of professional training.

Concepts of self-assessed professional competency and self-efficacy have two major aspects in common that relate to the present study. First, both concepts require an individual to engage in self-evaluation. Second, both self-assessed competency and self-efficacy impact levels of actual competence (Bradley et al., 2012), self-concept (Hughes et al., 2011), and effectiveness of the therapeutic process (Fauth \& Williams, 2005). Because of their association with one another, it appears that self-efficacy would be necessary to elicit accurate levels of self-assessed competence and that, alternatively, self-assessed competence is foundational for enhanced selfefficacy. Therefore, the present study accounts for both concepts and examines trainees' selfassessed levels of professional competency and counselor self-efficacy as outcome variables. 
Although the field of psychology's current culture of competence emphasizes the importance of producing competent professional psychologists, little is known about how to improve competency (Ridley et al., 2011). The focus of competency is on the observable behavior of the trainee or clinician. This exposes a problem with the competency standards; the APA along with faculty and supervisors are expecting a certain level of competency and outcome without providing much, if any, detail about how to acquire developmentally appropriate competency. In response, the present study examines two variables, mindfulness and self-compassion, as they relate to outcomes associated with competency and self-efficacy.

Mindfulness and self-compassion were chosen as predictor variables in this study due to their profound effects on self-evaluation and perspective on self (e.g., Gockel et al., 2013; Greason \& Cashwell, 2009; Ruggiero et al., 2014). Mindfulness is defined in two complimentary ways. First, mindfulness as a process is termed mindful practice, which involves practicing intentional attention without judgment. Second, mindfulness as an outcome is termed mindful awareness and involves a deep understanding of one's present moment (Shapiro, 2009). Selfcompassion is similar in that this concept also involves self-awareness and nonjudgment. Neff (2003b) describes self-compassion as an act of opening oneself to understand one's own suffering and to heal oneself with kindness rather than criticism or judgment. Because both constructs encompass self-awareness, it is likely that mindfulness and self-compassion are related to self-efficacy and, in turn, associated with self-assessed competence. The present study will assess mindfulness and self-compassion and their associations with counselor self-efficacy and self-assessed professional competence in professional psychology with the aim of providing training programs with strategies to improve competence in their trainees through implementation of these concepts. 


\section{Professional Competency in Training Programs}

Epstein and Hundert (2002) broadly describe professional psychology competence as the "habitual and judicious use of communication, knowledge, technical skills, clinical reasoning, emotions, values, and reflection in daily practice for the benefit of the individual and community being served" (p. 226). More concisely, researchers conceptualize competencies in the field of psychology as consisting of three main components: knowledge, skills, and attitudes (Kaslow et al., 2004; Peterson et al. 1992). Thus, each competency required of a professional psychologist must include aspects related to each of these three components.

During the 2002 competencies conference, Rodolfa et al. (2005) introduced The Cube Model (Figure 1) which conceptualized the development of competencies throughout the various stages of training and learning, ranging from professional education through lifelong learning stages. This model proposed 12 core competencies that are conceptualized as foundational or functional. Foundational competencies involve the knowledge, skills, attitudes, and values that "serve as the foundation for the functions a psychologist is expected to perform" (e.g.., awareness and understanding of individual and cultural diversity issues, etc.; Fouad et al., 2009, p. S6). Functional competencies integrate these foundational competencies and describe the primary functions that a psychologist is expected to perform (e.g., assessment, intervention, etc.). In addition to the functional and foundational core competencies, the Cube Model (Rodolfa et al, 2005) includes the various stages of professional development (e.g., doctoral education, doctoral internship, etc.). It is important to note that all three dimensions (i.e., Functional, Foundational, and Stage of Development) are not mutually exclusive and, instead, overlap with one another. Refer to Figure 1, which illustrates the Cube Model that assesses both functional and 
foundational domains as well as stages of development regarding trainee competence in professional psychology (Rodolfa et al., 2005).

\section{Competency Cube**}

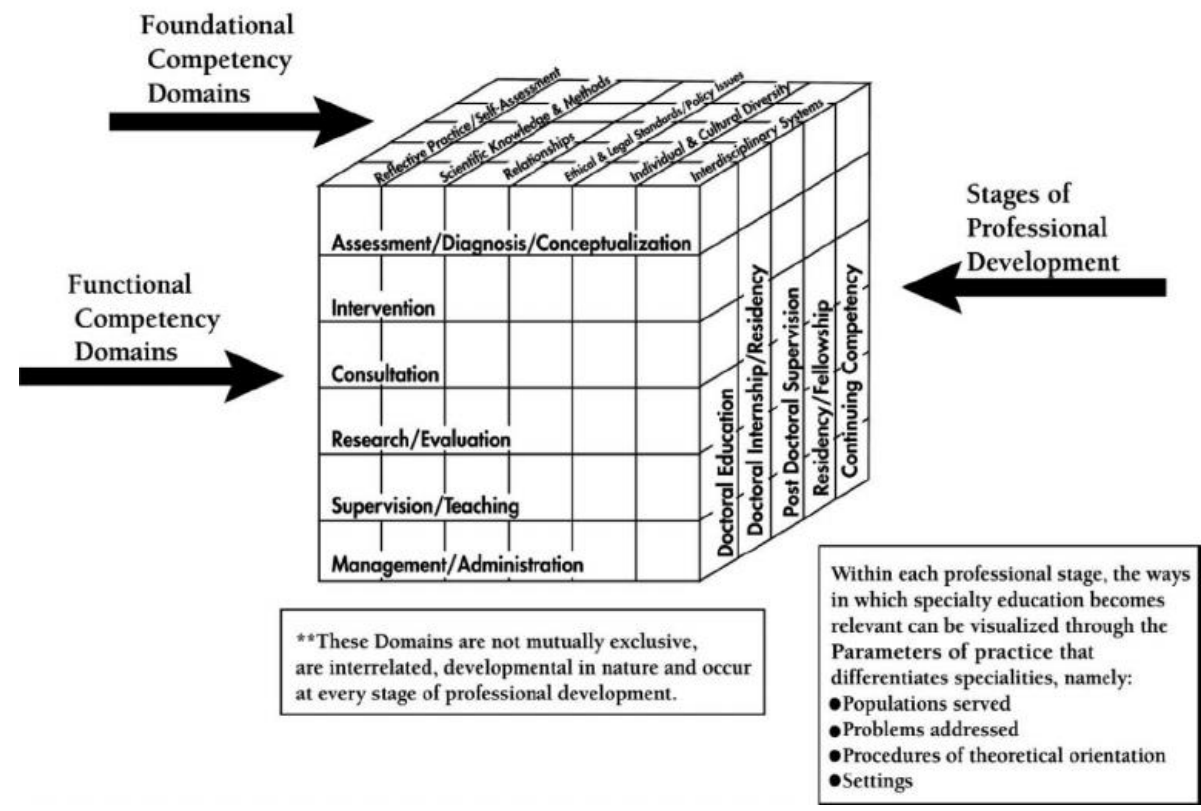

Figure 1. Competency Cube Model (Rodolfa et al., 2005).

The field of professional psychology explored ways to cohesively and comprehensively define and measure trainee learning outcomes across all accredited institutions (Fouad et al., 2009). Licensing boards also had a vested interest in requiring documentation from trainees to prove they met the competencies outlined by the field. Thus, standards of competency needed to be developed to meet the needs of the various stakeholders. The current competency standards were updated in 2011, titled the Revised Competency Benchmarks for Professional Psychology (APA, 2011). These standards both identify each competency that must be met by the trainee and provide an opportunity for the evaluator to describe the acquisition of each competency through the sequences of training (Fouad et al., 2009). With this document, training and academic institutions can objectively and holistically assess their trainees as they move forward in each level of their training. 


\section{Professional Competencies Benchmark Standards}

The Revised Competency Benchmarks (APA, 2011) include "core professional competencies and competency benchmarks across three levels of professional development: readiness for practicum, readiness for internship, and readiness for entry to practice" (Fouad et al., 2009, p. S6). As mentioned above, there are 15 total core competencies, 8 of which are functional competencies (assessment, intervention, consultation, research and evaluation, supervision, teaching, administration, and advocacy) and 7 of which are foundational competencies (professionalism, reflective practice, scientific knowledge and methods, relationships, individual and cultural diversity, ethical and legal standards and policy, and interdisciplinary systems; APA, 2011). These standards are used by training directors within educational settings to assess trainees' readiness at various stages of their professional development within their doctoral program.

Another critical aspect of doctoral-level training within counseling and clinical psychology is in the applied realm of working with clients at practicum placements and internship sites. During this process, licensed supervisors use the APA benchmark standards to evaluate their trainee's level of competence in working with counseling diverse clientele. The applied aspects of counseling entail many cognitive, behavioral, and affective components of therapy.

Because of the shift toward a culture of competence (Roberts et al., 2005), more research is being published regarding ways that the benchmarks standards help to assess and measure competencies in training programs across the nation. As a field, professional psychology is improving ways to implement the benchmark standards in their education and training programs to increase levels of competency in current and future graduating classes of psychologists. 
However, the field continues to be less familiar with what factors contribute to achieving and obtaining competency in the above-listed 15 core domains. The present study will address this gap in the literature by further validating the benchmark standards while also exploring the relationships between specific practices (i.e. mindfulness, and self-compassion) of trainees and their levels of competence and self-efficacy.

\section{Self-Assessment of Professional Competency}

It is imperative that practicing psychologists maintain competence as an essential principle in the codes of ethics governing the general body of professional psychology. Competency in the field of psychology is a complex concept which includes not only attaining and maintaining the knowledge, skills, and abilities appropriate for professional psychological work, but also includes positive self-assessment of competence. In other words, beyond the general competency factors, practicing psychologists and psychology trainees should own a sense of self-assessed professional competence that is appropriate for their developmental level. Researchers have examined elements involved in acquiring self-assessed competence in practicing psychologists, including engaging in professional reading, taking appropriate coursework, having multiple years of experience, and participating in conferences and conventions (Bradley et al., 2012). However, few, if any, literature exists to help understand selfassessment of competency in psychology trainees.

Belar et al.'s (2001) article examining self-assessment in clinical health psychology illustrates one of the only models of integrating self-assessment within the field of professional psychology. The researchers offer a self-assessment template for practicing psychologists, which includes 13 questions for practitioners to ask themselves to assess their competence in working specifically with clients that present with health-related concerns (Belar et al., 2001). This model 
is comprehensive in that it attends to knowledge, skills, and attitudes, the three essential components of competency assessment. After conducting a thorough review of the literature to date, this article appears to be the sole article that proposes a template and model for implementing self-assessment strategies to measure competency within the field of professional psychological practice. Unfortunately, this model is only applicable to practicing psychologists working with a specific subset of clientele.

Moreover, few, if any, studies have investigated how self-assessed professional competence is assessed and achieved in psychology trainees. Rather, the competency literature is primarily geared toward use and applicability for a population of practicing psychologist. Recognizing this gap in the literature, Roberts and colleagues (2005) published a call to action to the field of professional psychology stating, "the field itself needs to develop further appropriate models for self-assessment" (p. 356). The researchers argue that the procedures for assessing competence in trainees should involve both self-assessment and external assessment, where the emphasis is placed on students to learn how to adequately and accurately assess their own skill development throughout their training and career as psychologists (Roberts et al., 2005).

Through indirect methods many researchers address the importance of self-assessed professional competency throughout training within the field of psychology. Lamb et al. (1982) argue that an enhanced sense of competence and independence within practice is essential to doctoral-level interns as they navigate their way through internship year and into their career as professional psychologists. Further, Belar and colleagues (2001) discuss how essential accurate perceptions of one's competency are regarding the ethical standards practicing psychologists observe. The researchers highlight the general ethical code of competency, emphasizing that it requires frequent self-regulation and self-assessment to act within the limits of expertise and 
maintain knowledge and awareness of advances within the field (Belar et al., 2001). For these reasons, there is a high need for self-assessed professional competency to be examined within the psychology literature, particularly with trainee populations.

Though self-assessed professional competency has not yet been identified as a key construct within the general domain of training and competency, other constructs, such as selfefficacy, show significant associations with self-assessed professional competency and can assist in filling this gap in the literature. Hughes and colleagues (2011) demonstrated a significant, positive relationship between self-efficacy and competency-related self-concept within a sample of high school students. Furthermore, the researchers reported a hierarchical relationship between these two constructs, where levels of self-efficacy impacted levels of competency as it related to individuals' self-concept. This finding is important to consider given that self-efficacy is an internal experience whereas the qualities outlined by Bradley et al. (2012) exhibit more external qualities as it relates to competency (i.e. professional reading, coursework, etc.). By understanding that internal attributes, such as self-efficacy, impact levels of self-assessed competency, training programs across the nation can better serve their students and, in turn, produce more effective and competent clinicians.

As trainees are evaluated through the APA's benchmark standards, I argue that it is equally important that trainees use the same standards to assess their own levels of competence. Thus, for this study, trainees were asked to assess their levels of self-assessed professional competence using a shortened, validated, and reliable version of the APA's benchmark standards that measures the functional and foundational competency domains. In addition, considering that self-assessed competency and self-efficacy have been shown to be associated with one another, 
this study will further assess this relationship by including a measure of self-efficacy that specifically applies to the psychology trainee population.

\section{Counselor Self-Efficacy}

Self-efficacy, as defined by Bandura (1977), is the degree to which individuals consider themselves capable of performing a particular task or activity. In counseling, this concept is important because of its relation to the benchmark standards outlined above. Similar to the selfefficacy definition provided by Bandura, counselor self-efficacy refers to the belief that a clinician has the ability to perform required counseling competencies and produce successful outcomes (Larson \& Daniels, 1998). It is important to note that the literature often uses the terms counselor self-efficacy and counseling self-efficacy interchangeably with both of these constructs having been defined similarly. The current paper focuses on counselor self-efficacy, as it is defined above, and uses this term throughout the entirety of the paper.

Considering that each trainee is being assessed by their faculty through use of the competency benchmarks, it is critical that the trainee have a degree of self-efficacy in each functional and foundational domain for them to succeed. In fact, self-efficacy within the field of professional psychology has been of interest for decades; so much so that several measures of counselor self-efficacy (e.g., Counseling Self-Efficacy Scale, Counseling Self-Estimate Inventory, Interpersonal Skills Efficacy Scale, Counselor Behavioral Evaluation- Self-Efficacy) have been developed and validated for use with trainee populations in their clinical and academic work.

Throughout the literature, counselor self-efficacy has been shown to be associated with insight- and outcome-related variables. For example, various research studies indicate that counselor self-efficacy is associated with intrapersonal traits, such as anxiety and self-thought 
(Al-Darmaki, 2004; Friedlander et al., 1986), as well as being aware of one's values (Larson et al., 1992). Additionally, counselor self-efficacy has been shown to be related to levels of performance and effectiveness in clinical work, such as use of advanced clinical skills (Greason \& Cashwell, 2009; Larson \& Daniels, 1998), attending to process, dealing with difficult client behaviors, and behaving in culturally competent ways (Larson et al., 1992).

These findings are important when considering the impact that self-efficacy has on clinical performance in trainees; however, it is important to note that the majority of these studies indicate inconsistent relationships between counselor self-efficacy and level of training. For example, several researchers indicate that counselor self-efficacy increases simultaneously with advancement through an academic training program (i.e., Friedlander \& Snyder 1983; Goreczny et al. , 2015; Margolies et al., 1986; Melchert et al., 1996; O’Brien et al., 1997). On the other hand, other researchers have argued that the relationship between these two constructs is nonlinear and only increases slightly following the initial stages of training (i.e., Johnson \& Seem, 1989). Further, Sipps et al. (1988) stated that variations in counselor self-efficacy differed based on the course of academic and applied training rather than solely measuring differences across progression through the academic program. Larson and Daniels (1998) posited that these inconsistent results may be due to the utilization of different measures of counselor self-efficacy in the methodology of these studies. Another reason for this inconsistency may be that the researchers have studied self-efficacy in relation to self-assessed performance without the inclusion of other, potentially influential, positive intrapersonal aspects within the trainee. The present study explores how positive traits (i.e., mindfulness and self-compassion), rather than negative (e.g., anxiety), impact levels of counseling-self efficacy and self-assessed professional competency. 
Researchers have argued that counselor self-efficacy is an "essential element in preparing graduate students to become professional counselors" (Ruggiero et al., 2014, p. 2). In fact, due to the influx of literature describing the importance of counselor self-efficacy in the clinical domain, educators and supervisors have expressed interest in discovering ways to increase selfefficacy among counseling trainees (Al-Darmaki, 2004; Kozina et al., 2010; Sipps et al., 1988; Urbani et al., 2002). Melchert and colleagues (1996) suggested that professional training, education, and supervised experience are all ways to improve counselor self-efficacy in graduate students. This assertion provides a foundation for the present study as the researchers investigated the variance in self-efficacy of doctoral-level trainees at differing levels of their training. In addition, the researchers explored the influence of the positive traits of mindfulness and self-compassion on counselor self-efficacy and self-assessed professional competence.

\section{Self-Assessed Competency and Counselor Self-Efficacy: Contrasting Constructs}

Both competency and self-efficacy have been examined throughout the literature when discussing variables related to counselor performance. In fact, many researchers argue that "there are not clear conceptual and empirical distinctions between the constructs of counselor selfefficacy and counseling self-perceptions" (Constantine, 2002, p. 83). Researchers suggest that both of these constructs have the potential to be inaccurately associated with performance and the provision of effective service delivery (Lent et al., 1998). Therefore, it is important that the constructs be clearly differentiated for the purpose of the current study.

The first area of distinction between self-assessed competency and counselor selfefficacy lies within the definition of these constructs. As noted above, counselor self-efficacy refers to counselors' beliefs or judgements about their ability to work effectively with in their clinical work (Larson \& Daniels, 1998). On the other hand, self-assessed competency is more 
broadly defined as a clinician's assessment of their attitudes, beliefs, knowledge, and skills in working with clients. Taking these two definitions into account, it becomes clear that selfefficacy and self-assessed competency are evaluated at different moments of a clinician's experience. That is to say, counselor self-efficacy is a cognitive assessment completed by a clinician prior to their performance of a behavior and self-assessed competency is a clinician's evaluation of their cumulative performance up until that point. Furthermore, Larson (1998) explains "outcome expectations and goals and plans, seem to be present when the counselor session begins... Self-evaluation seems to occur primarily at the end of counseling” (pp. 230231). Thus, it is important to include these two constructs and variables in the present study to examine both the trainee's beliefs about their expected performance as well as their assessment of their present performance.

Another area of distinction between these variables is related to clinicians' beliefs about their performance. In general, counselor self-efficacy is associated with specific behaviors or skills, whereas self-assessed competency consists of beliefs performance within specific dimensions of training, such as self-awareness (Constantine \& Ladany, 2001). Bong and Skaalvik (2003) state that "efficacy judgment is less concerned with what skills and abilities individuals possess. It considers more important what individuals believe they can do with whatever skills and abilities they may possess" (p. 5). Therefore, when assessing self-efficacy, researchers use measures, such as with the Counselor Self-Efficacy Scale (Melchert et al., 1996), that highlight a set of outlined skillsets specific to a desired behavior. Alternatively, when assessing self-assessed competency, researchers utilize measures, such as the Professional Competency Scale-Revised (Taylor, 2015), that include broad and general items that reflect required abilities of an efficient and competent clinician. Both the Counselor Self-Efficacy Scale 
and Professional Competency Scale-Revised were used in the present study as a way to effectively measure these two distinct constructs.

\section{Mindfulness and Self-Compassion}

The variables of mindfulness and self-compassion have been researched alongside one another frequently throughout the literature. The literature in these domains exhibits uncertainty as to whether self-compassion is considered an outcome of mindfulness or a component of it (Baer et al., 2006). This finding is not surprising considering mindfulness has been identified as a component of self-compassion and is itemized on various measures of self-compassion (e.g., Self-Compassion Scale; Neff, 2003a; Self-Compassion Scale - Short Form; Raes et al., 2011) used in research.

Neff (2003b) argues that mindfulness is both a predictor and criterion of self-compassion, in that mindfulness is a necessary component of self-compassion and that it can be enhanced with greater levels of self-compassion. Additionally, the researchers note that both constructs involve a level of acceptance toward painful experiences (Neff \& Dahm, 2015). Thus, it is not surprising that many research findings discuss a positive relationship between mindfulness and self-compassion (Baer et al., 2012; Kabat-Zinn, 2003; Neff, 2003b). Consequently, researchers often include both variables in studies as they explore effects on outcome variables (Fulton, 2016; Fulton \& Cashwell, 2015).

\section{Mindfulness}

The construct of mindfulness has been researched for more than 25 years and continues to be explored within the field of counseling psychology. Mindfulness is rooted in the Buddhist tradition and is defined as "the awareness that emerges through paying attention on purpose, in the present moment, and nonjudgmentally to the unfolding of experience moment by moment" 
(Kabat-Zinn, 2003, p. 145). Though mindfulness is commonly associated with the literature produced by Kabat-Zinn, Langer (1989) initially introduced the concept and theory of mindfulness as associated with interpersonal interaction. Langer's (1989) interest in studying mindfulness stemmed from her research on mindlessness, which she defined as "minimal information processing" (p. 138). Accordingly, the researcher described mindfulness as both a cognitive and an affective state where an individual is active in the processing of information obtained from their environment (Langer, 1989). More recent literature has broadened the definition of mindfulness to include two components. The first component involves regulating one's attention to remain present in the immediate experience, and the second consists of one's openness to their approach to engage in the experience (Bishop et al., 2004). Through the decades of research on this concept, cognition and affect have remained as key processes that collaboratively describe a mindful state of being.

The practices and emphases regarding mindfulness vary considerably. In Eastern traditions, mindfulness practice takes the form of various types of meditation, such as Theravada, Mahayana, and Vajrayana (Kabat-Zinn, 2003). These practices differ based on geographical regions of Eastern Asia and utilize various schools of thought, sub-traditions, and texts to support their practice. The general concept of mindfulness practice in Eastern traditions is viewed as a "skillful understanding of how unexamined behaviors and what Buddhists would call an untrained mind can significantly contribute directly to human suffering, one's own and that of others" (Kabat-Zinn, 2003, p. 146). Human suffering, then, is transformed through meditative practices into enhanced clarity of the mind, openness of the heart, and a refining of attention and action. 
Within the past half-century, the Western world has adopted Eastern traditions of mindfulness meditation in various degrees. Though the roots of meditative practice continue to exist in the Westernized version of mindfulness, equal attention is geared toward producing insight and engaging in compassion practices. In this way, mindfulness is considered both an outcome and a process (Shapiro, 2009). As a process, mindful practice is described as "the systematic practice of intentionally attending in an open, caring, and discerning way, which involves both knowing and shaping the mind" (Shapiro, 2009, p. 556). As an outcome, mindfulness is considered an awareness where an individual experiences a deep understanding "that manifests as freedom of mind" (Shapiro, 2009, p. 556).

As a process, mindfulness is practiced in the form of meditation. Westernized formal mindfulness meditation techniques typically include body scan meditations, sitting meditations, hatha yoga, walking meditations, and loving-kindness meditations (Jain et al., 2007). Each of these techniques integrates cognitive, somatic, and spiritual components that encourage individuals to regulate their attention and emotions, focusing both intrapersonally and interpersonally (Shapiro \& Schwartz, 2000). This type of meditation cultivates a sense of nonjudgmental, present awareness within the individual (Jain et al., 2007). For example, Jain and colleagues (2007) found that students who participated in a mindfulness meditation intervention across four weeks reported significant reductions in overall psychological distress, including somatization, obsessive-compulsive traits, interpersonal sensitivity, depression, anxiety, hostility, phobic anxiety, paranoid ideation, and psychoticism.

As an outcome, mindfulness practice has been proven effective to alleviate feelings of distress. For example, a study conducted by Shapiro et al. (1998) illustrated that participation in a mindfulness-based stress reduction intervention resulted in reduced self-reports of depression 
and anxiety in a sample of premedical and medical students. Orzech et al. (2009) found reductions in anxiety-related symptoms in a sample of community adults who participated in mindfulness training. In a sample of health care professionals, Shapiro et al. (2005) showed that involvement in a mindfulness-based stress reduction (MBSR) intervention throughout a span of eight weeks resulted in decreased levels of perceived stress, psychological distress, and job burnout. Altogether, the results of these studies exemplify the outcome effects that mindfulness practice has on various levels of distress in a range of diverse populations.

In counselor training, mindfulness is both a process and an outcome as it is exhibited through the trainee's ability to attend more closely within session and engage in openness and acceptance during interactions with their client (Bruce et al., 2010; Fulton, 2016; Morgan \& Morgan, 2005). Mindfulness also impacts trainees' ability to be objective about their experiences; this concept is known as reperceiving, wherein an individual increases their capacity for objectivity in their relationship with their own internal and external experiences (Shapiro et al., 2006). Trainees without this level of awareness have reported increased levels of doubt, anxiety, and self-consciousness; increased mental blocks and distractions; and critical, negative thoughts relating to their work (Friedlander et al., 1986; Hiebert et al., 1998; Williams et al., 1997). The rapid growth of research in this area of study has led to introductions of various interventions and implications in clinical training and practice, such as an instructional strategy within educational psychology (i.e., Stoops, 2005), a practical framework within sport psychology (i.e., McAlarnen, 2015), and a therapeutic intervention in clinical practice (i.e., Goldin \& Gross, 2010). 
Similar to mindfulness, the concept of self-compassion originated in Buddhist philosophy and became a topic of interest in Western psychology over a decade ago under the research of Kristin Neff. Neff (2003b) expressed interest in exploring alternative constructs to self-esteem as indicators of healthy self-concept. Prior to this time, the literature on self-evaluation relied heavily on the use of self-esteem as a variable to measure psychological health in this domain. Researchers faced various challenges using self-esteem as a sole measure of self-evaluation, such as its effects on distorting self-perception and self-knowledge (Baumeister et al., 1993) and on altering how individuals think about others in comparison to themselves (Feather, 1994). For these reasons, researchers and psychologists initiated the movement toward finding alternative conceptualizations of ways individuals create a healthy relationship with oneself. Concepts such as self-respect (Seligman, 1995), true self-esteem (Deci \& Ryan, 1995) and personal character (Damon, 1995) were introduced in the literature in the mid-1990s. While studying Buddhist philosophy one decade later, Neff (2003b) incorporated self-compassion into her research resulting in the expansion of literature in the general domain of self-evaluation.

Neff (2003b) defined self-compassion as “being touched by and open to one's own suffering, not avoiding or disconnecting from it, generating the desire to alleviate one's suffering and to heal oneself with kindness" (p. 87). The act of self-compassion involves "offering nonjudgmental understanding to one's pain, inadequacies and failures, so that one's experience is seen as part of the larger human experience" (Neff, 2003b, p. 87). Thus, having self-compassion engages individuals in viewing themselves through a lens of self-acceptance in which they understand that their own experiences are also those of a common human experience. Simply put, self-compassion is compassion turned toward oneself regarding any perceived aspect of suffering that one may experience (Neff, 2016). Aspects of suffering may include painful 
thoughts and emotions, dealing with hardships, and experiencing personal failures (Neff, 2003b; Neff \& Dahm, 2015).

The construct of self-compassion includes various components. First, it involves being kind and understanding to oneself when one perceives themselves as inadequate. Second, it entails the understanding that failure and pain are unavoidable in all humanity. Last, it involves a balanced awareness of one's emotional state with the ability to confront painful thoughts and feelings (Neff, 2003b). Neff (2003b) described these components to represent a specific frame of mind, where each aspect is viewed on a spectrum that ranges from uncompassionate to compassionate behavior.

The three main components of self-compassion include self-kindness versus selfjudgment, a sense of common humanity versus isolation, and mindfulness versus overidentification (Neff, 2016). Consistent with the theoretical framework of mindfulness, selfcompassion is exemplified in cognitive and affective ways. Self-kindness reflects the affective components of self-compassion and involves providing oneself with warmth and unconditional acceptance through being gentle, supportive, and understanding. This component is expressed when one soothes themselves in times of distress. On the opposite side of the spectrum, selfjudgment entails being critical and harsh toward oneself for any shortcomings (Neff, 2016). Common humanity represents the cognitive component of self-compassion and refers to having a shared experience with all humans, with the recognition that mistakes are inevitable and universally experienced. This aspect is expressed when individuals hold a more broad and connected perspective regarding the challenges and difficulties they face. On the other hand, isolation can occur when individuals feel alone in their imperfections and hold a mindset that is more egocentric (Neff, 2016). Lastly, mindfulness, in relation to self-compassion, entails 
cognitive and affective awareness of the present moment. This component manifests when individuals express clarity and balance toward themselves in moments of suffering. Alternatively, overidentification is typically seen when individuals become consumed with negative aspects of themselves or their experiences (Neff, 2016).

Similar to mindfulness, self-compassion practices can be completed formally or informally. Programs such as Mindful Self-Compassion (MSC; Neff \& Germer, 2013) have been developed as formal methods of teaching self-compassion. These types of programs typically consist of meditative practices that emphasize kindness and love toward self. Types of informal practices involve deep breathing techniques and use of positive mantras throughout one's daily routine. For this study and due to the absence of a formalized training program, the informal practice methods of self-compassion and mindfulness are of focus.

\section{Contrasting Constructs}

Though there are several clear commonalities between these constructs, it is important to note the distinctions between mindfulness and self-compassion for clarity in this study. First, the constructs are both broader than what is typically captured in the literature. As a whole, mindfulness is a more comprehensive concept than what is termed mindfulness within the construct of self-compassion (Neff \& Dahm, 2015). Mindfulness refers to the ability to attend to one's experiences with a sense of composure; whereas, mindfulness as a component of selfcompassion is narrower in its scope and focuses purely on awareness of negative thoughts and feelings (Neff \& Dahm, 2015). Alternatively, the construct of self-compassion is broader in scope in that mindfulness is only one of its three components. The two additional components, self-kindness and common humanity, are not necessarily active aspects of mindfulness. Neff and 
Dahm (2015) argue that these components are more intentional in self-compassion exercises comparative to the passivity of these aspects in mindfulness practice.

Another difference between mindfulness and self-compassion relates to the entity that is being targeted through these practices. Mindfulness approaches are geared toward relating to the internal experiences of an individual, whereas self-compassion techniques attend to the individual as a whole experiencing distress (Germer, 2009). Thus, when an individual experiences a sense of suffering, they can be mindful about their thoughts, emotions, and sensations in the present moment and also be compassionate toward themselves, expressing care and concern to their being (Neff \& Dahm, 2015). Though the act of expression may overlap with one another, they can be mutually exclusive. In fact, Neff and Dahm (2015) argue that selfcompassion and mindfulness mutually enhance one another, where both constructs are necessary to achieve greater levels of well-being. Neff and Dahm (2015) state, "mindfulness is necessary to ensure that compassion doesn't become a slick new form of resistance... while compassion provides the emotional safety needed to fully feel and open [up] to one's pain" (p. 129). For these reasons, the present study investigates both self-compassion and mindfulness independently and in relation to variables related to training in a sample of doctoral-level psychology students.

Taken altogether, it is important to examine both mindfulness and self-compassion as distinct constructs in the present study because they equally contribute value to the purpose of the study. In the present study, mindfulness is examined as a way of being in the present moment and attending to one's experience nonjudgmentally. Self-compassion is examined in the current study as the way one responds and reacts to their experiences and perceives challenge and hardship. Both constructs are necessary in the present study because of their value in assessing 
traits of trainees specific to that of their mindful attentiveness and self-compassionate responses. Though the construct of self-compassion has mindfulness as an associated variable, it is important that the present study examine mindfulness separately and distinctly to more broadly understand the variance attributed to both constructs.

Further, the present study includes separate and distinct measures to assess selfcompassion and mindfulness. The Self-Compassion Scale Short-Form (SCS-SF; Raes et al., 2011) was used to assess a trainee's degree of self-compassion. Trainees were asked to reflect on their experiences and respond to items that measure self-kindness, self-judgment, common humanity, isolation, mindfulness, and over-identification. This scale was specifically chosen for the present study because it distinctly assesses self-compassion in individuals as it relates to their experiences with failure and inadequacy. On the other hand, the Mindful Attention Awareness Scale (Brown \& Ryan, 2003a) was used to assess trainee's attention to and awareness of present moment experiences. This measure of mindfulness is different than the subscale measure of mindfulness on the SCS-SF because individuals were asked to reflect on their everyday emotional, cognitive and behavioral experiences. Using these measures, the present study more clearly differentiates self-compassion and mindfulness as it relates to competency and selfefficacy in trainees.

\section{Mindfulness and Self-Compassion in Training}

As both similar and distinct concepts, mindfulness and self-compassion have been examined throughout the literature on training in graduate programs. However, much of the literature attends to the relationships of these constructs with indirect variables related to training, such as counselor anxiety and use of empathy. In general, both counselor anxiety and empathy are variables associated with counselor preparation and performance in applied settings 
(Fulton, 2016; Fulton \& Cashwell, 2015). First, from the theoretical standpoint, empathy is a critical component in the development of the therapeutic alliance (Rogers, 1957). Second, empathy has been associated with positive treatment outcomes as rated by client report and supervisor ratings of client improvement (Elliott et al., 2011). Within the past decade, literature has illustrated a positive, significant relationship between mindfulness and empathy among counseling trainees at various levels in training (Greason \& Cashwell, 2009; Leppma \& Young, 2016; Morgan \& Morgan, 2005). Similarly, researchers have identified the positive relationship among mindfulness, self-compassion, and empathy in samples of health care professionals and within the general community (Birnie et al., 2010; Neff \& Pommier, 2013; Raab, 2014).

Whereas empathy is a positive therapeutic intervention, anxiety is often viewed as a negative and detrimental factor that impacts trainees' preparation and performance in their applied work. Independently, self-compassion and mindfulness were negatively correlated with anxiety and rumination in a sample of hospital patients, (Farb et al., 2010), college students (Bergen-Cico \& Cheon, 2014; Van Dam et al., 2010), counseling psychology graduate students (Fulton \& Cashwell, 2015), as well as the general population (e.g., Birnie et al., 2010; Neff, 2003b). Positive relationships have been reported among self-compassion, mindfulness, and general well-being in a sample of counseling trainees (May \& O’Donovan, 2007), which is important considering counselor well-being is associated with counseling effectiveness (Beutler et al., 2004). Through examining the literature, it is clear that a relationship exists among these constructs; however, the literature is limited to these findings and does not explore potential path analyses to other outcome-related variables.

To address this gap in the literature, researchers have studied ways mindfulness strategies have impacted counselor performance through both quantitative and mixed method research 
designs. Training in mindfulness techniques has generated interest as a way to improve counselor performance. For example, students in social work and marriage and family therapy programs indicated that mindfulness practices enhanced their ability to be more attentive to clients during session and to draw better boundaries around their work in practicing self-care (Gockel, et al., 2013; McCollum \& Gehart, 2010). Further, trainees who engaged in consistent mindfulness practice reported higher levels of counseling skill development compared to those who did not engage in any form of practice (Buser et al., 2012; Gockel et al., 2013). In a mixed methods study, one trainee described the importance of practicing mindfulness and its effect on managing personal reactions “so they don’t play out unconsciously” (Gockel et al., 2013, p. 349), evidencing potential correlations between mindfulness, intrapersonal awareness, and multicultural competency (Ivers et al., 2016). Lastly, in a study assessing client-reported outcomes, trainees' self-report of mindfulness practice was positively associated with clients' perceptions of empathy during the session (Fulton, 2016) and positive treatment outcomes (Grepmair et al., 2007). These findings suggest that mindfulness practice can be a foundation of enhanced counselor performance; however, this assumption cannot be validated without appropriately measuring performance based on the standards of competency that are set forth in accredited training programs. Additionally, in theory, self-compassion is associated with aspects of training, such as goal-setting, goal-striving, and insight into one's limitations of their performance (Neff, 2003b), though no empirical evidence of this relationship exists. Thus, a gap in the literature remains.

In addition to counselor performance, researchers have also investigated relationships among mindfulness, self-compassion, and counselor self-efficacy in attempt to further explore outcome-related variables. Throughout the literature, mindfulness practice has been shown to 
predict greater levels of self-efficacy in counseling trainees through direct methods whereas the research on self-compassion is limited to indirect associations. Regarding mindfulness, Gockel et al. (2013) found that trainees who spent more time practicing mindfulness experienced greater increases in overall counselor self-efficacy from pre-test to post-test $(r=0.320, p=0.018)$. Greason and Cashwell (2009) substantiated these findings and noted that in their study mindfulness accounted for up to $20 \%$ of the overall variance in counselor self-efficacy reported by trainees. On the other hand, self-compassion has been shown to be related to greater levels of counselor self-efficacy through indirect associations. For example, Ruggiero and colleagues (2014) indicated that self-compassion among other "healthy personal adjustment" variables increased the prediction of counselor self-efficacy by $9 \%$ above and beyond variables attributed to professional development (p. 9). These findings suggest that self-compassion has a relationship with counselor self-efficacy, though the assumptions are limited to understanding the effect size in context with the other variables self-compassion was categorized with in this study. As noted above, self-compassion is negatively correlated with anxiety (e.g., Fulton \& Cashwell, 2015). This finding is important when considering that anxiety is negatively correlated with counselor self-efficacy (Larson \& Daniels, 1998). Though mindfulness and self-compassion are often viewed as interchangeable concepts, these empirical findings show the variance within publications examining these constructs in relation to counselor self-efficacy and general counselor performance.

As summarized above, the findings of these empirical studies reflected that practices of both mindfulness and self-compassion have implications for training through direct and indirect means. Thus, most of the research in this area details implications for training. However, methods used in previous studies for investigating the relationships among these variables only 
assess for characteristics of the trainee rather than variables involved in training. Understanding the relationship between different facets of training (e.g., competency and self-efficacy) and the practice of mindfulness and self-compassion has yet to be explored in more detail. Thus, the limitations in the above studies call for research to examine the relationship among these constructs. With an alternative and comprehensive method relative to the above-cited studies, the present study explores this gap in the literature and addresses implications for training by assessing variables of mindfulness, self-compassion, self-assessed professional competence, and counselor self-efficacy.

\section{Purpose of the Study}

The aims of the present study were to investigate the relationships among mindfulness, self-compassion, counselor self-efficacy and self-assessed professional competence. Current research evidence shows that anxiety and low self-esteem have an inverse relationship with perceived self-efficacy in counseling psychology trainees (Goreczny et al., 2015). In addition, counseling trainees' self-efficacy beliefs were positively related to their self-assessed competence in counseling diverse clients (Constantine, 2002). Therefore, interventions and implications that can foster self-efficacy and promote self-assessed professional competence may lead to enhanced counseling performance.

Researchers have suggested that mindfulness is related to increased self-compassion (Neff, 2003b), reductions in anxiety (Feltman et al., 2009) and, consequently, improvements in self-efficacy (Greason, \& Cashwell, 2009). Given the established independent relationships among mindfulness and self-compassion, the present study explores how these constructs are related to self-assessed professional competency and counselor self-efficacy in a sample of clinical and counseling psychology doctoral-level trainees. 


\section{Research Questions and Hypotheses}

The study surveyed mindfulness and self-compassion in relation to self-assessed competency in training and counselor self-efficacy, and investigated the following research questions:

\section{Research Question One}

While controlling for demographic variables, do increased levels of mindfulness, as measured by the MAAS, predict higher levels of self-compassion, as measured by the SCS-SF, in doctoral trainees?

Hypothesis 1. It is expected that there will be a significant, positive relationship between levels of mindfulness and self-compassion, such that participants who report high levels of mindfulness will also endorse high levels of self-compassion.

\section{Research Question Two}

Do increased levels of mindfulness, as measured by the MAAS, and self-compassion, as measured by the SCS-SF, uniquely predict higher levels of counselor self-efficacy, as measured by the CSES, in doctoral trainees?

Hypothesis 2. While controlling for self-compassion, it is expected that there will be a significant, positive relationship between levels of mindfulness and counselor self-efficacy, such that participants who report high levels of mindfulness will also endorse high levels of counseling self-efficacy.

Hypothesis 3. While controlling for mindfulness, it is expected that there will be a significant, positive relationship between levels of self-compassion and counselor self-efficacy, such that participants who report high levels of self-compassion will also endorse high levels of counselor self-efficacy. 


\section{Research Question Three}

Do increased levels of mindfulness, as measured by the MAAS, and self-compassion, as measured by the SCS-SF, uniquely predict higher levels of self-assessed professional competency, as measured by the PCS-R, in doctoral trainees?

Hypothesis 4. While controlling for self-compassion, it is expected that there will be a significant, positive relationship between levels of mindfulness and self-assessed professional competency, such that participants who identify high levels of mindfulness will also endorse high levels of self-assessed professional competency.

Hypothesis 5. While controlling for mindfulness, it is expected that there will be a significant, positive relationship between levels of self-compassion and self-assessed professional competency, such that participants who identify high levels of self-compassion will also endorse high levels of self-assessed professional competency.

\section{Research Question Four}

Do doctoral trainees differ uniquely in their levels of mindfulness, as measured by the MAAS, and self-compassion, as measured by the SCS-SF, when taking into account training level?

Hypothesis 6. While controlling for self-compassion, it is expected that there will be a significant, positive relationship between trainee level and scores on measures of mindfulness, such that participants with more advanced training will report higher levels of mindfulness than those who indicate lower trainee status in their program.

Hypothesis 7. While controlling for mindfulness, it is expected that there will be a significant, positive relationship between trainee level and scores on measures of self- 
compassion, such that participants with more advanced training will report higher levels of selfcompassion than those who indicate lower trainee status in their program. 


\section{Design}

\section{CHAPTER 2: METHOD}

The present study is a cross-sectional, survey, correlational design with two predictor variables (mindfulness and self-compassion) and two outcome variables (self-assessed professional competency and counselor self-efficacy). A total of five measures were administered to participants, one for each variable and an additional demographics measure. The four measures for the identified variables were counterbalanced to reduce the potential of order effects, with the demographics measure remaining constant as the final measure of the survey.

\section{Participants}

Participants in the current study were recruited through email solicitation to training directors at APA-accredited institutions that house a counseling psychology, clinical psychology, or combined counseling and clinical psychology Ph.D. or Psy.D. program, as well as training directors at APA-accredited internship sites. To gain access to this population, the investigator used the Association of Psychology Postdoctoral and Internship Centers (APPIC, n.d.) website to search for APA-accredited pre-doctoral internship sites and the APA (n.d.) website to search for eligible training programs. All potential participants were provided with a description of the study, documentation of the Institutional Review Board (IRB) approval (Appendix C), and a link to the survey.

Participants were required to have met the following criteria: at least 18 years of age; currently enrolled in a doctoral-level (Ph.D. or Psy.D.) counseling psychology, clinical psychology, or combined counseling-clinical psychology program that is APA-accredited; and actively involved in a practicum or internship experience at an APA-accredited site. These criteria were included in the survey, and surveys completed by individuals who did not meet these criteria were not considered in the data analysis. 
An a priori power analysis was conducted using G*Power 3.0.10 (Faul et al., 2007) to determine an appropriate sample size for the study. The analysis was computed using a medium effect size of 0.15 (Cohen, 1988), two predictor variables (mindfulness and self-compassion), and statistical power of 0.80 and resulted in a minimum sample size of 68 participants. After reviewing the literature that included the variables and measures in this study, a medium effect size was chosen. The research in this area described discrepancies in effect size with a number of studies highlighting a small effect size (i.e., Jain et al., 2007; Gockel et al., 2013; Orzech et al., 2009), various studies reporting a large effect size (i.e., MacBeth \& Gumley, 2012; Neff \& Dahm, 2015; Osman et al., 2016; Ying, 2009), and majority of studies not including the effect size (i.e, Neff et al., 2005; Neff et al., 2007; Shapiro et al., 2005; Shapiro et al., 2006; Shapiro et al., 1998). Considering the variance in the effect sizes within this area of the literature, a medium effect size was chosen for this study to best represent the findings in the literature and to reduce any potential concerns related to data analysis. In addition, the minimum sample size was increased to reflect recommendations by Cook, Heath and Thompson (2000) who indicated an average response rate of 39.6\% $(S D=19.6 \%)$ in web-based survey research. To account for unexplained variance, potential drop out (Hoerger, 2010), a minimum of 180 participants was established in order to account for an estimated response rate of 38\%. After obtaining 196 participants for the present study, G*Power was used to determine the minimum effect size the study could achieve with statistical power of 0.80 . The analysis computed a $f^{2}$ of 0.05 , which is a small effect size (Cohen, 1988).

\section{Measures}

\section{Demographic Questionnaire}


An 8-item demographic questionnaire was created by the investigator to gather background information about the participants of the study. Participants were asked to provide information about their age, gender identity, ethnicity, degree, year in program, primary practicum/internship site, type of doctoral program, and engagement in various mindfulness and self-compassion practices. See Appendix D for a detailed description of the items and response options. Descriptive statistics were reported for each of these eight variables in addition to their inclusion as controls in the regression equations used for data analysis.

\section{Mindful Attention Awareness Scale (MAAS; Brown \& Ryan, 2003a)}

The Mindfulness Attention Awareness Scale (MAAS; Brown \& Ryan, 2003a) consists of 15 items designed to assess an individual's attention to, and awareness of, present moment experiences. Participants are asked to rate their responses regarding their daily experiences on a 6-point Likert scale, ranging from 1 (almost always) to 6 (almost never) with 3 representing somewhat frequently. An example item is, "It seems I am 'running on automatic' without much awareness of what I'm doing." Considering all test items are worded negatively, the response items are ranked accordingly. Total scores were obtained by summing item responses. Scores on this measure range from 15 to 90, with higher scores reflecting greater levels of mindfulness. See Appendix E for a detailed description of the measure items and response options.

In the original scale validation study, Brown and Ryan (2003b) demonstrated evidence for convergent, discriminant and criterion validity. The MAAS has been shown to be moderately correlated to the Trait Meta-Mood Scale $(r=0.46)$ and the Mindfulness/Mindlessness Scale $(r=$ $0.31)$, and modestly related to the NEO-PI subscale of openness to experience $(r=0.18)$. The researchers found a weak association between the MAAS and multiple social desirability scales and self-consciousness (Brown \& Ryan, 2003b). Further, Brown and Ryan (2003b) reported the 
MAAS to be significantly related to several criterion measures of well-being, such as the Beck Depression Inventory $(r=-0.40)$, Profile of Mood States: Anxiety $(r=-0.34)$, Pleasant Affect $(r$ $=0.38)$, Unpleasant Affect $(r=-0.38)$, Positive and Negative Affect Schedule: Positive Affect $(r$ $=0.36)$, Positive and Negative Affect Schedule: Negative Affect $(r=-0.41)$, and Rosenberg SelfEsteem $(r=0.44)$.

Brown and Ryan (2003b) also identified test-retest reliability (4-week period between time 1 and time 2 ; intraclass correlation $=0.81 ; p<0.01)$ and internal consistency $(\alpha=0.87)$. In recent literature, researchers demonstrated a Cronbach's alpha of 0.89 when using this measure with graduate-level master's and doctoral psychology students (Robins et al., 2015). This measure is an appropriate instrument for the study because of its strong psychometric properties. Additionally, the constructs in this measure emphasize trait mindfulness, as opposed to state mindfulness, and outcome mindfulness, as opposed to process mindfulness, both of which are of focus in the study. In the present study, the MAAS (Brown \& Ryan, 2003a) demonstrated good internal consistency reliability $(\alpha=0.86)$, which is consistent with the results of previous studies using similar sample characteristics (i.e., Robins et al., 2015).

It is important to note that the MAAS (Brown \& Ryan, 2003a) has received some criticism by researchers who question whether this measure adequately measures the construct of mindfulness. In fact, the majority of mindfulness measures have been critiqued and called into question regarding their validity of adequately assessing mindfulness. For instance, Grossman (2011) argued that current self-report instruments, including the MAAS, have been validated by individuals untrained in proper mindfulness practices and, thus, these measurements do not reflect the aspects of mindfulness as defined in original Buddhist practice. In addition, a recently published study described how majority of self-report instruments that measure mindfulness 
include various flaws that cause concern about the content validity (Van Dam et al., 2018).

Given these considerations have been introduced in the literature more recently, there has not been an instrument developed that adequately addresses these concerns. Therefore, the MAAS was used in the current study because, to date, it has been the most widely used measure of mindfulness and because it differentiates mindfulness from self-compassion (Van Dam et al., 2018).

\section{Self-Compassion Scale Short-Form (SCS-SF; Raes et al., 2011)}

The Self-Compassion Scale-Short Form (SCS-SF; Raes et al., 2011) contains 12 items that measure participants' degree of self-compassion. The SCS-SF has six subscales: selfkindness (2 items), self-judgment ( 2 items), common humanity (2 items), isolation ( 2 items), mindfulness ( 2 items), and over-identified ( 2 items). Participants are asked to rate how often they behave in the manners as indicated by each of the items on a 5-point Likert scale ranging from 1 (almost never) to 5 (almost always). An example item is, "I try to see my failings as part of the human condition." Total scores were obtained by adding subscale item responses while considering reverse scores from the negatively worded subscale items. On this measure, negative subscales that will be reverse-scored included self-judgment, isolation, and over-identification. The total scores range from 12 to 60, with higher scores indicating greater levels of selfcompassion. See Appendix F for a detailed description of the measure items and response options.

The SCS-SF was developed as a shortened and reliable version of the original SCS (Raes et al., 2011). The SCS-SF showed a correlation of 0.97 with the original, long-form SCS. The long-form SCS evidenced high internal consistency and construct validity $(\alpha=0.92$; Neff, 2003b). The SCS-SF was initially validated on a sample of 415 college students and exhibited 
high internal consistency reliability $(\alpha=0.86)$. Raes and colleagues $(2011)$ reported excellent goodness-of-fit through confirmatory factor analyses, supporting the unidimensional structure of the measure. Internal consistency values of 0.84 and 0.87 were found at two data collection points in a sample of first-year psychology students (Raes, 2011). Test-retest reliability (5-month interval from time 1 to time 2) for the SCS-SF in this sample was 0.71 . In recent literature using the SCS-SF with doctoral counseling psychology student participants, researchers found a Cronbach's alpha of 0.86 , suggesting this measure to be valid with this population (Palcan, 2011). The SCS-SF is an appropriate measure to use in the present study because it has been shown to be valid and reliable (Neff, 2003b; Palcan, 2011; Raes et al., 2011). In the present study, the SCS-SF (Raes et al., 2011) demonstrated good internal consistency reliability ( $\alpha=$ 0.86), which is consistent with the results of previous studies using similar sample characteristics (i.e., Palcan, 2011).

\section{Counselor Self-Efficacy Scale (CSES; Melchert et al., 1996)}

The Counselor Self-Efficacy Scale (CSES; Melchert et al., 1996) measures the degree of confidence a counselor has in their counseling abilities. The CSES is a 20-item measure that examines both knowledge- and skill-based competencies related to the practice of individual and group counseling. Participants are asked to rate their counseling abilities using a 5-point Likert scale ranging from 1 (agree strongly) to 5 (disagree strongly), with 3 representing neutral or uncertain. An example item is, "I can effectively facilitate client self-exploration." Total scores were obtained by summing response items after reverse-scoring ten identified items. The total score ranges from 20 to 100, with higher scores reflecting greater levels of counselor selfefficacy. 
In the original construct validation of the CSES, researchers reported a Cronbach's alpha coefficient of 0.91 and a test-retest reliability (1-week interval between time 1 and time 2) of 0.85 (Melchert et al., 1996). Melchert et al. (1996) tested for convergent validity and reported a correlation $(r=0.83)$ between the CSES and the Self-Efficacy Inventory. Reliability analysis in more recent literature with a doctoral-level psychology student sample evidenced strong reliability ( $\alpha=0.87$; Unsworth, 2015), which is consistent with previous literature. This measure is appropriate for the present study because it has consistent reliability and validity statistics. Additionally, this measure was useful in identifying participants' perceptions of their counseling abilities and knowledge competencies while involved in an applied practicum or internship experience. In the self-efficacy literature, this measure appears to be overwhelmingly utilized with master's level clinicians. Therefore, using this instrument in the present study added to the reliability of this measure with a doctoral-level sample. In the present study, the CSES (Melchert et al., 1996) demonstrated good internal consistency reliability $(\alpha=0.84)$, which is consistent with the results of previous studies using similar sample characteristics (i.e., Unsworth, 2015).

\section{Professional Competencies Scale-Revised (PCS-R; Taylor, 2015)}

The Professional Competency Scale-Revised (PCS-R; Taylor, 2015) was developed as a self-assessment of professional competencies within the field of applied psychology. The PCS-R is a shortened format comparative to the original survey. The revised scale consists of 19 items across three subscales, including Foundational Competencies, Functional Competencies, and Continuing Competencies. For the purpose of the present study and applicability of the sample, only the foundational and functional subscales were used. The sample used for the present study included psychology trainees who do not meet the criteria to be evaluated on continuing competencies benchmarks (i.e., practicing psychologists). Thus, only 16 items were used from 
this measure for this study. The Foundational Competencies subscale is further broken into five scales as follows: Professionalism (2 items); Reflection Practice/Self-Assessment/Self-Care (2 items); Scientific Knowledge and Methods (2 items); Relationships (2 items); and Interdisciplinary Systems ( 2 items). The Functional Competencies subscales include the following three scales: Assessment (2 items), Intervention ( 2 items), and Consultation ( 2 items). Participants are asked to identify their agreement with each item on a 5-point Likert scale ranging from 1 (strongly disagree) to 5 (strongly agree), with 3 representing neither agree nor disagree. An example item is, "I select and implement interventions appropriate for the presenting issue(s)." Total scores were obtained by summing item responses. Total scores for the two subscales range from 16 to 80 , with higher scores indicating greater levels of self-assessed professional competencies in academic and applied settings within the field of counseling psychology.

The PCS-R was originally validated with a sample of 428 psychologists and produced a Cronbach's alpha coefficient of 0.84 (Taylor, 2013). The Cronbach's alpha coefficient of the Foundational Competencies subscale was 0.76 and was shown to be related to the Therapist SelfCare Scale $(r=0.16)$ and the Miville-Guzman Universality-Diversity Scale-Short Form ( $r=$ 0.41). The Functional Competencies subscale had a Cronbach's alpha of 0.82 and was correlated to the Counselor Self-Efficacy Scale $(r=0.55)$. The above listed correlated measures and the addition of the Jefferson Scale of Psychotherapist Lifelong Learning were instruments used to measure the convergent validity of the PCS-R (Taylor, 2013).

Although the PCS-R has not been retested in recent literature, a recent study indicated a Cronbach's alpha coefficient of 0.88 when an abbreviated version of the PCS long-form was used with a sample of 136 psychologists (Taylor \& Neimeyer, 2015). The PCS-R is an 
appropriate measure to use in the present study because it assesses areas of both knowledge- and skill-based competencies relative to the competencies outlined by the APA. Evaluating doctorallevel trainees on these competency domains is an essential component of training within the psychology disciplines as they are applied to students across various levels of their development (i.e., readiness for practicum, readiness for internship, readiness for practice). Considering this instrument is newly developed, the present study further validated this measure with a doctorallevel psychology trainee population. In the present study, the PCS-R (Taylor, 2015) demonstrated good internal consistency reliability $(\alpha=0.84)$.

\section{Procedure}

Approval to conduct the study was obtained from West Virginia University's IRB. The recruitment letter and URL of the survey was sent to training directors from eligible APAaccredited internship sites and doctoral training programs. Participants were able to view an informational page detailing the aim of the study, information regarding the researchers' educational institution and contact information, informed consent (Appendix A), confidentiality procedures, eligibility requirements, time allotment, and information about the option to receive a gift card post-completion of the survey. Participants were also informed that they may discontinue and withdraw from the study at any time. Participants were then asked to indicate their willingness to participate before completing the questionnaires.

After consenting to participate in the survey, eligible individuals completed the entire survey which consisted of five pages of questionnaires, each page including one of the four identified measures (CSES, Melchert et al., 1996; MAAS, Brown \& Ryan, 2003a; PCS-R, Taylor, 2015; SCS-SF, Raes et al., 2011) with an additional page consisting of the demographic questionnaire items. To reduce the potentiality of priming effects, the measures were set to 
alternate in presentation for each participant and the demographic information was held constant to appear as the last page of the survey. Upon completion of the survey, participants were directed to an independent URL that provided resources to assist in reducing any distress that may have been triggered by participating in the study. Participants were also given the option to enter their email address to receive a $\$ 5$ gift card after completion of the study. To maintain privacy and confidentiality, participants were informed that their email addresses would not be associated with their survey responses.

\section{Data Analyses}

Data collected in the present study was analyzed using the IBM Statistical Package for the Social Sciences (SPSS) Statistics (2015) program.

\section{Preliminary Analyses}

Preliminary analyses were conducted to assess the data for normality and statistical assumption violations of the primary analyses. Descriptive statistics, means, standard deviations, and scale reliabilities were calculated on all measures. A bivariate correlation matrix was calculated to test the relationships among the main study variables (mindfulness, selfcompassion, counselor self-efficacy, and self-assessed competency) and with the demographic variables. Demographic variables that were significantly associated with the main study variables were included as covariates in the primary analyses.

There are a number of statistical assumptions pertaining to multiple linear regression that were attended to in the preliminary analyses. Specifically, multiple linear regression assumptions include linearity, multivariate normality, multicollinearity, and homoscedasticity. Linearity was tested through use of scatterplots. Normality was checked using a histogram and goodness of fit test to determine whether errors between observed and predicted values were normally 
distributed. Multicollinearity was checked through use of a correlation matrix to determine whether correlations existed between the predictor variables (mindfulness and self-compassion). Homoscedasticity was tested by creating a scatterplot of residuals versus predicted values to determine whether a pattern in the distribution was detected (Judd et al., 2009). Overall, the data was tested to determine whether any statistical assumptions associated with multiple linear regression were violated.

\section{Primary Analyses}

The following analyses were used to test each of the eight hypotheses of this study:

Hypothesis 1. While controlling for covariates, participants who report high levels of mindfulness will also endorse high levels of self-compassion. This hypothesis was analyzed first with the bivariate correlation matrix and second with a stepwise linear regression. The predictor variable was mindfulness, as measured by the MAAS (Brown \& Ryan, 2003a), and the outcome variable was self-compassion, as measured by the SCS-SF (Raes et al., 2011). In the initial model, covariates were entered. In the second model, covariates were entered followed by average scores on the MAAS.

Hypothesis 2. While controlling for self-compassion and covariates, participants who report high levels of mindfulness will also endorse high levels of counselor self-efficacy. This hypothesis was analyzed using a stepwise linear regression. The predictor variable was mindfulness, as measured by the MAAS (Brown \& Ryan, 2003a). The outcome variable was counselor self-efficacy, as measured by the CSES (Melchert et al., 1996). In the initial model, covariates were entered. In the second model, covariates were entered followed by average scores of self-compassion. In the final model, covariates and average scores of self-compassion were entered followed by average scores on of mindfulness. 
Hypothesis 3. While controlling for mindfulness and covariates, participants who report high levels of self-compassion will also endorse high levels of counselor self-efficacy. This hypothesis was tested using a stepwise linear regression. The predictor variable was selfcompassion, as measured by the SCS-SF (Raes et al., 2011). The outcome variable was counselor self-efficacy, as measured by the CSES (Melchert et al., 1996). In the initial model, covariates were entered. In the second model, covariates were entered followed by average scores of mindfulness. In the final model, covariates and average scores of mindfulness were entered followed by average scores on of self-compassion.

Hypothesis 4. While controlling for self-compassion and covariates, participants who identify high levels of mindfulness will also endorse high levels of self-assessed professional competency. This hypothesis was analyzed using a stepwise linear regression. The predictor variable was mindfulness, as measured by the MAAS (Brown \& Ryan, 2003a). The outcome variable was self-assessed competency, as measured by the PCS-R (Taylor, 2015). In the initial model, covariates were entered. In the second model, covariates were entered followed by average scores of self-compassion. In the final model, covariates and average scores of selfcompassion were entered followed by average scores on of mindfulness.

Hypothesis 5. While controlling for mindfulness and covariates, participants who identify high levels of self-compassion will also endorse high levels of self-assessed professional competency. This hypothesis was analyzed using a stepwise linear regression. The predictor variable was self-compassion, as measured by the SCS-SF (Raes et al., 2011). The outcome variable was self-assessed competency, as measured by the PCS-R (Taylor, 2015). In the initial model, covariates were entered. In the second model, covariates were entered followed by 
average scores of mindfulness. In the final model, covariates and average scores of mindfulness were entered followed by average scores on of self-compassion.

Hypothesis 6. While controlling for self-compassion and covariates, participants with more advanced training will report higher levels of mindfulness than those who indicate lower trainee status in their program. This hypothesis was analyzed using a stepwise linear regression. The predictor variable was training year indicated by the participant on the demographic questionnaire. The outcome variable was mindfulness, as measured by the MAAS (Brown \& Ryan, 2003a). In the initial model, covariates were entered. In the second model, covariates were entered followed by average scores of self-compassion, as measured by the SCS-SF. In the final model, covariates and average scores of self-compassion were entered followed by average scores on of mindfulness.

Hypothesis 7. While controlling for mindfulness and covariates, participants with more advanced training will report higher levels of self-compassion than those who indicate lower trainee status in their program. This hypothesis was analyzed using a stepwise linear regression. The predictor variable was training year indicated by the participant in the demographic questionnaire. The outcome variable was self-compassion, as measured by the SCS-SF (Raes et al., 2011). In the initial model, covariates were entered. In the second model, covariates were entered followed by average scores of mindfulness, as measured by the MAAS. In the final model, covariates and average scores of mindfulness were entered followed by average scores on of self-compassion. 


\section{Respondent Demographics}

\section{CHAPTER 3: RESULTS}

Participants completed a series of online surveys using Qualtrics. Inclusion criteria, which were provided in the recruitment letter and on introduction page of the survey, stating that the participant must currently be 18 years or older; enrolled in an APA-accredited clinical psychology, counseling psychology, or combined counseling-clinical psychology program; and enrolled in an internship or practicum experience. Two hundred fifty-one surveys were submitted. Survey responses were removed for participants who did not meet the eligibility criteria, which resulted in a removal of 27 surveys. Specifically, two participants indicated enrollment in school psychology programs, one participant indicated being enrolled in their postdoctoral year, and 24 participants indicated that they were not currently enrolled in a practicum or internship experience. There were also several incomplete surveys that were considered for removal. Schafer (1999) recommends a maximum of 5\% cutoff of missing data before the data becomes problematic. Thus, incomplete surveys $(n=28)$ were listwise deleted for data analysis because the amount of cases with incomplete data represented $0.13 \%$ of the overall data values, which is well below the cutoff percentage. In addition, due to unequal sample size and significant variance in sampling, some demographic variables were condensed into two categories which resulted in deletion of survey responses. Specifically, gender and type of program were condensed into two categories. Of the total participants, only one identified being from a combined clinical and counseling program and three participants reported identifying as non-binary. Therefore, the total sample size for the study was 192, which demonstrated a $76.5 \%$ completion rate.

Participants included 158 women-identified (82.3\%) and 34 men-identified (17.7\%) doctoral-level students. Their ages ranged from 22 to 43 years with a mean age of 27.67. The 
ethnic breakdown of the sample was as follows: Caucasian American or White $(70.8 \% ; n=136)$, African American or Black (7.8\%; $n=15)$, Hispanic or Latinx $(7.3 \% ; n=14)$, Asian American or Pacific Islander $(5.7 \% ; n=11)$, Biracial or Multiracial $(4.2 \% ; n=8)$, Arabic American or Middle Eastern $(2.1 \% ; n=4)$, “Other: Not Listed” $(1 \% ; n=2)$, and East Indian or Indian American $(1 \% ; n=2)$. For the purposes of conducting preliminary analyses with these unequal sample sizes within ethnic breakdown, the ethnicity and racial categories were condensed into two categories (White (70.8\%) and Non-White (29.2\%)).

Participants indicated being enrolled in counseling psychology $(27.6 \% ; n=53)$ and clinical psychology $(72.4 \% ; n=139)$ programs. One hundred and thirty participants $(67.7 \%)$ reported being enrolled in a Ph.D. program and 62 participants (32.3\%) reported being enrolled in a Psy.D. program. Participants indicated the variance in distribution of current year in their program (see Table 1). Participants identified being involved at the following practicum or internship sites: university counseling center $(32.8 \% ; n=63)$, hospital or medical setting $(23.4 \%$; $n=45)$, community agency $(16.1 \% ; n=31)$, VA medical center $(11.5 \% ; n=22)$, department/campus clinic $(4.7 \% ; n=9)$, correctional facility $(2.6 \% ; n=5)$, primary school $(2.1 \% ; n=4)$, independent practice $(1.6 \% ; n=3)$, residential facility $(1.6 \% ; n=3)$, group practice $(1 \% ; n=2)$, consortium $(1 \% ; n=2)$, academic department $(1 \% ; n=2)$, and hospice $(0.5 \% ; n=1)$. With regard to regular mindfulness and self-compassion related practices, participants reported the following: self-compassion breaks $(33.3 \% ; n=64)$, relaxation exercises $(31.8 \% ; n=61)$, meditation $(23.4 \% ; n=45)$, mindful walking $(23.4 \% ; n=45)$, yoga $(22.4 \% ; n=$ $43)$, journaling/writing $(19.8 \% ; n=38)$, physical exercise $(14.3 \% ; n=28)$, mindful cooking/eating $(12 \% ; n=23)$, prayer $(3.6 \% ; n=7)$, art/music $(2 \% ; n=4)$, personal 
therapy/utilization of clinical skills $(2 \% ; n=4)$, martial arts $(1 \% ; n=2)$, crocheting $(1 \% ; n=2)$

and massage $(0.5 \% ; n=1)$. Table 1 provides a visual representation of participant demographics.

Table 1

Participant Demographics

\begin{tabular}{|c|c|c|}
\hline Variable & $\begin{array}{l}\text { Number of } \\
\text { Participants }\end{array}$ & $\%$ \\
\hline \multicolumn{3}{|l|}{ Gender } \\
\hline Man & 34 & $17.7 \%$ \\
\hline Woman & 158 & $82.3 \%$ \\
\hline \multicolumn{3}{|l|}{ Race/Ethnicity } \\
\hline Caucasian American/White & 136 & $70.8 \%$ \\
\hline $\begin{array}{l}\text { Non-Caucasian American/Non- } \\
\text { White }\end{array}$ & 56 & $29.2 \%$ \\
\hline \multicolumn{3}{|l|}{ Type of Program } \\
\hline Counseling Psychology & 53 & $27.6 \%$ \\
\hline Clinical Psychology & 139 & $72.4 \%$ \\
\hline \multicolumn{3}{|l|}{ Type of Degree } \\
\hline Ph.D. & 130 & $67.7 \%$ \\
\hline Psy.D. & 62 & $32.3 \%$ \\
\hline \multicolumn{3}{|l|}{ Year In Program } \\
\hline $1^{\text {st }}$ Year & 7 & $3.6 \%$ \\
\hline $2^{\text {nd }}$ Year & 38 & $19.8 \%$ \\
\hline $3^{\text {rd }}$ Year & 37 & $19.3 \%$ \\
\hline $4^{\text {th }}$ Year & 41 & $21.4 \%$ \\
\hline $5^{\text {th }}$ Year & 46 & $24.0 \%$ \\
\hline $6^{\text {th }}$ Year & 21 & $10.9 \%$ \\
\hline $7^{\text {th }}$ Year & 2 & $1.0 \%$ \\
\hline
\end{tabular}

Note. $N=192$.

\section{Preliminary Analyses}

\section{Descriptive Statistics}

Mindfulness, as measured by the MAAS, had a mean score of 57.64 (Range $=31$ to 84, $S D=10.68)$. Self-compassion, as measured by the SCS-SF, had a mean score of 38.68 (Range = 17 to $57, S D=7.87$ ). Counselor self-efficacy, as measured by the CSES, had a mean of 82.57 (Range $=60$ to $100, S D=8.71)$. Lastly, self-assessed professional competency, as measured by the PCS-R, had a mean of 66.82 (Range $=48$ to $80, S D=6.01)$. Additionally, Cronbach's alpha 
were calculated to measure the internal consistency reliability of each instrument. Results were as follows: MAAS $(\alpha=0.86)$, SCS-SF $(\alpha=0.86)$, CSES $(\alpha=0.84)$, and PCS-R $(\alpha=0.84)$.

Theses scores reflect similar scores to those of the original validation studies. Specifically, the validation studies reported the following scores: MAAS $(\alpha=0.87)$, SCS-SF $(M=36.00, S D=$ 7.33, $\alpha=0.86$; Raes et al., 2011), CSES ( $\alpha=0.91)$, and PCS-R $(\alpha=0.84$; Taylor, 2015). The validation studies of the MAAS (Brown \& Ryan, 2003b), CSES (Melchert et al., 1996) and PCSR (Taylor, 2015) did not report full scale means or standard deviations. The internal consistency for each scale fell within the "good" range based on Cronbach's alpha descriptive definitions (Cohen, 1988).

Two of the six demographic variables are continuous (age and year in program) and four of the variables are categorical (gender, ethnicity, type of program, and degree). Practicum site was not considered in the preliminary analysis because of the unequal sample size for the sites which resulted in large variation among overall practicum and internship site enrollment (e.g., university counseling center $n=65$ vs. hospice $n=1$ ). Table 2 illustrates the results of bivariate correlations indicating the associations among predictor, outcome and continuous demographic variables.

Table 2 Correlations Among Variables

\begin{tabular}{|c|c|c|c|c|c|c|}
\hline & 1 & 2 & 3 & 4 & 5 & 6 \\
\hline 1. MAAS & -- & & & & & \\
\hline 2. SCS-SF & $0.41 * *$ & -- & & & & \\
\hline 3. CSES & $0.23 * *$ & $0.25 * *$ & -- & & & \\
\hline 4. PCS-R & $0.22 * *$ & $0.31 * *$ & $0.57 * *$ & -- & & \\
\hline 5. Age & 0.04 & 0.12 & $0.23 * *$ & 0.09 & -- & \\
\hline 6. Year & 0.11 & $0.19 * *$ & $0.36^{* *}$ & $0.18 *$ & $0.43^{* *}$ & -- \\
\hline
\end{tabular}

Note. $N=192$; MAAS = Mindfulness Attention Awareness Scale; SCS-SF = Self-Compassion Scale Short-Form; CSES = Counselor Self-Efficacy Scale; PCS-R=Professional Competency Scale-Revised. Two-tailed tests of significance: $* * p<.01, * p<.05$. 
Categorical demographic variables were separated based on numbers of groups for each variable. Descriptive statistics for gender, type of program, ethnicity and degree were conducted using three independent samples $t$-tests. An independent-samples $t$-test was conducted to determine if there were differences between men and women in their levels of mindfulness, selfcompassion, counselor self-efficacy and self-assessed professional competency. There was a statistically significant difference in self-compassion and mindfulness scores between men and women, with men scoring higher than women for both measures (see table 3 ). The difference between genders on counselor self-efficacy $(p>0.05)$ and self-assessed professional competency $(p>0.05)$ were not statistically significant.

Table 3 Results of t-tests and Descriptive Statistics of Variables by Gender

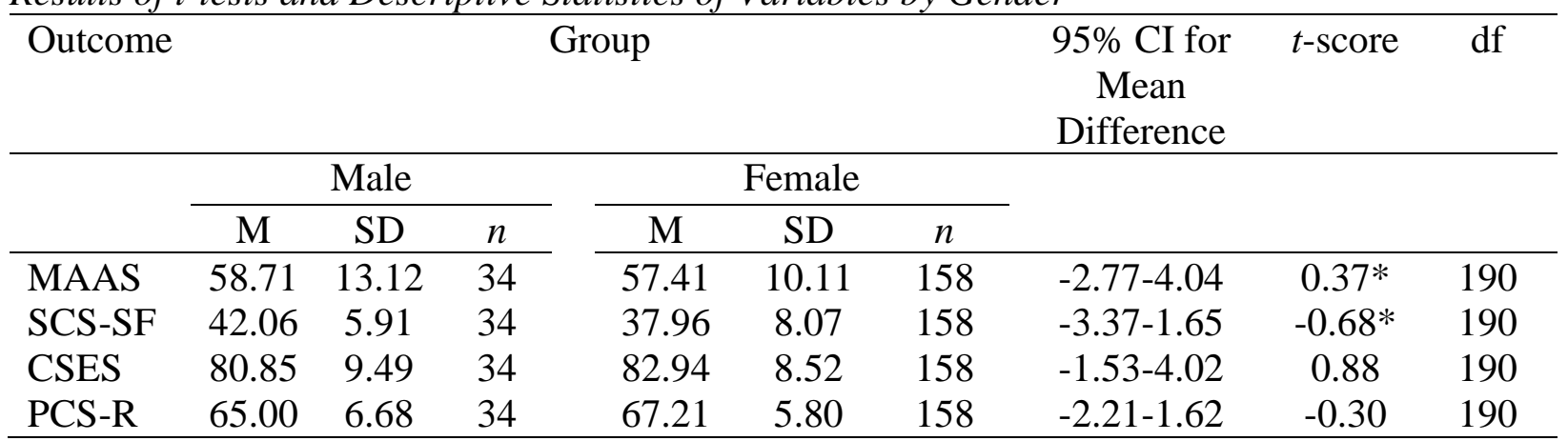
Note. $N=192 . * p<.05$.

A series of independent-samples $t$-tests were conducted to determine if there were differences between participants enrolled in a counseling psychology program or clinical psychology program in their levels of mindfulness, self-compassion, counselor self-efficacy and self-assessed professional competency. The difference between type of program on mindfulness $(p>0.05)$, self-compassion $(p>0.05)$, counselor self-efficacy $(p>0.05)$ and self-assessed professional competency $(p>0.05)$ scales were not statistically significant (see Table 4$)$. Similarly, independent-samples $t$-tests were run to determine if there were differences between participants enrolled in a Ph.D. program or Psy.D. program in their levels of mindfulness, self- 
compassion, counselor self-efficacy and self-assessed professional competency. The difference between type of degree on mindfulness $(p>0.05)$, self-compassion $(p>0.05)$, counselor selfefficacy $(p>0.05)$ and self-assessed professional competency $(p>0.05)$ scales were not statistically significant (see Table 5).

Table 4 Results of t-tests and Descriptive Statistics of Variables by Type of Program

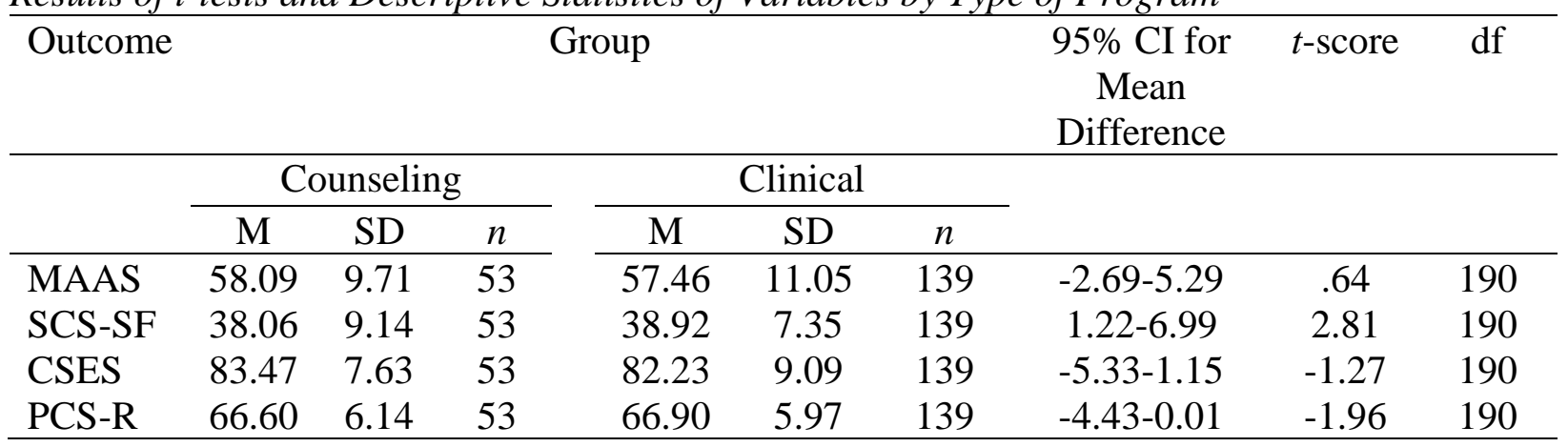

Note. $N=192$.

Table 5

Results of t-tests and Descriptive Statistics of Variables by Degree

\begin{tabular}{|c|c|c|c|c|c|c|c|c|c|}
\hline \multirow[t]{3}{*}{ Outcome } & \multicolumn{6}{|c|}{ Group } & \multirow{3}{*}{$\begin{array}{l}95 \% \text { CI for } \\
\text { Mean } \\
\text { Difference }\end{array}$} & \multirow[t]{3}{*}{$t$-score } & \multirow[t]{3}{*}{$\mathrm{df}$} \\
\hline & \multicolumn{3}{|c|}{ Ph.D. } & \multicolumn{3}{|c|}{ Psy.D. } & & & \\
\hline & $\mathrm{M}$ & SD & $n$ & $\mathrm{M}$ & SD & $n$ & & & \\
\hline MAAS & 58.05 & 10.71 & 130 & 56.77 & 10.65 & 62 & $-1.98-4.53$ & 0.77 & 190 \\
\hline SCS-SF & 39.00 & 7.87 & 130 & 38.02 & 7.90 & 62 & $-1.41-3.38$ & 0.81 & 190 \\
\hline CSES & 82.11 & 8.34 & 130 & 83.55 & 9.44 & 62 & $-4.09-1.21$ & -1.07 & 190 \\
\hline PCS-R & 66.75 & 6.16 & 130 & 66.97 & 5.72 & 62 & $-2.05-1.61$ & -0.24 & 190 \\
\hline
\end{tabular}

Note. $N=192$.

Lastly, an independent samples $t$-test was conducted to determine if mindfulness, selfcompassion, counselor self-efficacy and self-assessed professional competency were different for diverse ethnic groups. The difference between ethnic group on measures of mindfulness ( $p>$ $0.05)$, self-compassion $(p>0.05)$, counselor self-efficacy $(p>0.05)$ and self-assessed professional competency $(p>0.05)$ were not statistically significant. Considering these findings, 
gender identity, year in program and age will be considered as covariates for the primary

analyses.

Table 6

Results of t-tests and Descriptive Statistics of Variables by Race/Ethnicity

\begin{tabular}{|c|c|c|c|c|c|c|c|c|c|}
\hline \multirow[t]{3}{*}{ Outcome } & \multicolumn{6}{|c|}{ Group } & \multirow{3}{*}{$\begin{array}{l}\text { 95\% CI for } \\
\text { Mean } \\
\text { Difference }\end{array}$} & \multirow[t]{3}{*}{$t$-score } & \multirow[t]{3}{*}{ df } \\
\hline & \multicolumn{3}{|c|}{ White } & \multicolumn{3}{|c|}{ Non-White } & & & \\
\hline & $\mathrm{M}$ & SD & $n$ & M & SD & $n$ & & & \\
\hline MAAS & 57.80 & 10.87 & 136 & 57.96 & 10.29 & 56 & $-3.82-2.89$ & -0.27 & 190 \\
\hline SCS-SF & 38.43 & 8.06 & 136 & 39.30 & 7.44 & 56 & $-3.35-1.59$ & -0.70 & 190 \\
\hline CSES & 83.01 & 9.11 & 136 & 81.52 & 7.63 & 56 & $-1.24-4.22$ & 1.08 & 190 \\
\hline PCS-R & 67.16 & 5.83 & 136 & 65.98 & 6.38 & 56 & $-0.70-3.06$ & 1.24 & 190 \\
\hline
\end{tabular}

Note. $N=192$.

\section{Statistical Assumptions}

Prior to conducting primary analyses, the completed responses were evaluated across the following six assumptions: independence of observations, multicollinearity, linearity, homoscedasticity, no significant outliers, and normal distribution of residual errors.

Independence of Observations and Multicollinearity. To determine independence of observations, Durbin-Watson tests were conducted. There was an independence of residuals among all variables included in each regression model. Durbin-Watson statistics can range from 0 to 4 and scores closest to 2 indicate no correlation between residuals. The Durbin-Watson statistics were as follows: MAAS and SCS-SF (1.71), SCS-SF/MAAS and CSES (1.95), SCSSF/MAAS and PCS-R (2.34), year in program and MAAS (1.63), and year in program and SCSSF (1.71). Considering the Durbin-Watson scores for this study are between 1.63 and 2.34, it can be accepted that there is independence of residuals. To test for multicollinearity, regression analyses were conducted for all hypothesis. Coefficient tables for all analyses indicated a VIF between 1.00 and 1.29, which suggests that multicollinearity did not exist in the data. In addition, tolerance did not exceed 1.00 in any of the regression analyses. 
Linearity of Relationships and Homoscedasticity. Scatterplots were created to determine linearity and homoscedasticity by plotting studentized residuals against the predicted values. In addition, partial regression plots were reviewed to determine linear relationships between outcome and predictor variables. Predictor variables (mindfulness and self-compassion) were inputted in the $\mathrm{x}$-axis and outcome variables (counselor self-efficacy and perceived professional competency) were inputted in the y-axis so that both outcome variables were assessed for association with each predictor variable. Subsequently, year in program was inputted as a predictor variable and mindfulness and self-compassion were inputted as outcome variables to create two separate scatterplots. Visual inspection of scatterplots indicated a linear relationship between all the variables. In addition, homoscedasticity was demonstrated, as assessed by visual inspection of a plot of studentized residuals versus unstandardized predicted values. See Appendix I for figures related to linearity and homoscedasticity.

Data Normality and Outliers. To assess normality of each variable, normal probability plots were created, including histograms and Normal P-P Plots (See Appendix I). Each variable exhibited normal distribution of residuals through visual inspection of each plot. To detect outliers, casewise diagnostics, studentized deleted residuals, leverage points, and influential points (Cook's distance) were reviewed. There were 2 total outliers in the regression analyses: one on the CSES and one on the PCS-R. All outliers were not considered data entry error or measurement error. Rather than transforming the variables and to avoid further statistical assumption violations, the investigators conducted each regression analysis with and without the outliers to determine whether appreciable differences in the results were indicated. Considering there were no appreciable differences when excluding the outliers, the outliers were maintained and the results reflect findings using the complete data set. 


\section{Data Analyses}

Prior to conducting primary analyses, Pearson correlation analysis was used to determine associations among continuous variables (Table 2). Results indicated statistically significant relationships among all variables (mindfulness, self-compassion, counselor self-efficacy and self-assessed professional competency). There was a medium positive correlation between mindfulness and self-compassion. Thus, higher levels of mindfulness were associated with increased levels of self-compassion. There was a small positive association between mindfulness and counselor self-efficacy, suggesting that higher levels of mindfulness were related to increased levels of counselor self-efficacy. There was a small positive association between mindfulness and self-assessed professional competency, suggesting that higher levels of mindfulness were related to increased levels of self-assessed professional competency. Further, there was a small positive association between self-compassion and counselor self-efficacy, such that higher levels of self-compassion were related to greater levels of counselor self-efficacy. There was a medium positive association between self-compassion and self-assessed professional competency, suggesting that higher levels of self-compassion were related to increased levels of self-assessed professional competency. Lastly, there was a large positive association between counselor self-efficacy and self-assessed professional competency, such that higher levels of counselor self-efficacy were related to greater levels of self-assessed professional competency.

\section{Hypothesis 1}

Hypothesis one stated that while controlling for covariates, participants who reported high levels of mindfulness would also endorse high levels of self-compassion. To test this hypothesis, a bivariate correlation was conducted initially to determine associations between the 
variables. Results of the bivariate correlation (Table 2) indicated that year in program and age were covariates to be considered in the primary analysis. Further, results of an independent samples $t$-test indicated that gender would also be a covariate to account for in the primary analysis. The results of the bivariate correlation analysis suggested that average scores of mindfulness, as measured by the MAAS, and average scores of self-compassion, as measured by the SCS-SF, indicated a statistically significant positive relationship, such that higher values of mindfulness were associated with increased levels of self-compassion.

Following, a stepwise linear regression was conducted to determine if the addition of mindfulness improved the prediction of self-compassion above and beyond the covariates. Model 1 of the regression included the covariates (year in program, age and gender) as predictor variables and average scores of self-compassion, as measured by the SCS-SF, as the outcome variable. Model 2 included the covariates followed by average scores on mindfulness, as measured by the MAAS, as predictor variables and average scores of self-compassion as the outcome variable.

Results indicated that mindfulness statistically significantly predicted self-compassion above and beyond that of year in program, age and gender. Average scores of mindfulness accounted for $22 \%$ of the variance in self-compassion with adjusted $R^{2}=20.6 \%$. The differences in $R^{2}$ for the models $\left(f^{2}=0.28\right)$ indicated a medium effect size according to Cohen $(1988)$. The addition of mindfulness to the prediction of self-compassion (Model 2) also led to a statistically significant increase in $R^{2}$ of 0.15 . These findings indicate that scores on the MAAS were positively predictive of scores on the SCS-SF. See Table 7 for full details on each regression model. 
Table 7

Stepwise Linear Regression Predicting Self-Compassion from Covariates and Mindfulness

\begin{tabular}{|c|c|c|c|c|c|c|c|c|}
\hline \multirow[b]{3}{*}{ Variable } & \multicolumn{8}{|c|}{ Self-Compassion } \\
\hline & \multicolumn{4}{|c|}{ Model 1} & \multicolumn{4}{|c|}{ Model 2} \\
\hline & B & SE B & $\beta$ & $95 \% \mathrm{CI}$ & B & SE B & $\beta$ & $95 \% \mathrm{CI}$ \\
\hline Constant & 41.09 & 6.09 & & 29.09-53.09 & 24.57 & 6.23 & & $12.27-36.86$ \\
\hline Year & 0.97 & 0.43 & $0.18^{*}$ & $0.12-1.81$ & 0.73 & 0.40 & 0.13 & $-0.05-1.51$ \\
\hline Gender & -3.96 & 1.46 & $-0.19 * *$ & $-6.83-(-1.08)$ & -3.60 & 1.34 & $-0.18 * *$ & $-6.24-(-0.96)$ \\
\hline Age & 0.04 & 0.20 & 0.02 & $-0.36-0.44$ & 0.05 & 0.19 & 0.02 & $-0.32-0.42$ \\
\hline Mindfulness & & & & & 0.29 & 0.05 & $0.39 * *$ & $0.19-0.38$ \\
\hline$R^{2}$ & 0.07 & & & & 0.22 & & & \\
\hline$F$ & $4.96 * *$ & & & & $13.38^{* *}$ & & & \\
\hline$\Delta R^{2}$ & 0.07 & & & & 0.15 & & & \\
\hline$\Delta F$ & $4.96 * *$ & & & & $35.88^{* *}$ & & & \\
\hline
\end{tabular}

Note. $N=192 ; * * p<.01,{ }^{*} p<.05$

\section{Hypotheses 2 and 3}

Hypotheses two and three state that mindfulness and self-compassion will independently predict counselor self-efficacy while controlling for one another within regression models. To test these hypotheses, a stepwise linear regressions was conducted to determine the significance of the prediction among the variables. Model 1 of the regression included the covariates (year in program, gender and age) as predictor variables and average scores of counselor self-efficacy, as measured by the CSES, as the outcome variable. Model 2 included the covariates and average scores of mindfulness, as measured by the MAAS, as predictor variables, and average scores of counselor self-efficacy as the outcome variable. Model 3 included covariate variables, average scores of mindfulness, and average scores of self-compassion, as measure by the SCS-SF, as predictor variables and average scores of counselor self-efficacy as the outcome variable.

Results indicated statistical significance of the full model of covariates, self-compassion, and mindfulness to predict counselor self-efficacy. Average scores of self-compassion accounted for $20.9 \%$ of the variance in counselor self-efficacy with adjusted $R^{2}=18.7 \%$. Average scores of mindfulness accounted for $18.8 \%$ of the variance in counselor self-efficacy with adjusted $R^{2}=$ 
$17.0 \%$. The differences in $R^{2}$ for the models $\left(f^{2}=0.27\right)$ indicated a medium effect size according to Cohen (1988). The addition of mindfulness to the prediction of counselor self-efficacy (Model 2) led to a statistically significant increase in $R^{2}$ of .04 . The addition of self-compassion to the prediction of counselor self-efficacy (Model 3) led to a statistically significant increase in $R^{2}$ of .02. In Model 3, the significance of mindfulness predicting counselor self-efficacy disappears. These findings indicated that scores on the SCS-SF were positively predictive of scores on the CSES above and beyond mindfulness and covariates. These findings also suggest that scores on the MAAS were not predictive of scores on the CSES above and beyond other variables. See Table 8 for full details on each regression model.

The coefficients table illustrates a significant association between mindfulness and counselor self-efficacy in Model 2 which disappears in Model 3 after adding self-compassion. This effect suggests that self-compassion may be a mediating variable between the association of mindfulness and counselor self-efficacy. To test this assumption, a mediation model was conducted using Baron and Kenny's (1986) four step model. The first step of the model uses a linear regression analysis to determine associations between the predictor variable (mindfulness) and the outcome variable (counselor self-efficacy). The second step of the model uses a linear regression to determine associations between the predictor variable (mindfulness) and the suspected mediator variable (self-compassion). The third step of the model uses a linear regression to determine associations between the mediator variable (self-compassion) and the outcome variable (counselor self-efficacy). The final step of the model uses a linear regression to establish the mediation, illustrating that the predictor variable (mindfulness) is associated to the outcome variable (counselor self-efficacy) through the mediator variable (self-compassion). 
Table 8

Stepwise Linear Regression Predicting Counselor Self-Efficacy from Covariates, Mindfulness and Self-Compassion

\begin{tabular}{|c|c|c|c|c|c|c|c|c|c|c|c|c|}
\hline \multirow[b]{3}{*}{ Variable } & \multicolumn{11}{|c|}{ Counselor Self-Efficacy } & \multirow[b]{3}{*}{$95 \% \mathrm{CI}$} \\
\hline & \multicolumn{3}{|c|}{ Model 1} & \multicolumn{4}{|c|}{ Model 2} & \multicolumn{4}{|c|}{ Model 3} & \\
\hline & $\mathrm{B}$ & SE B & $\beta$ & $95 \% \mathrm{CI}$ & B & SE B & $\beta$ & $95 \% \mathrm{CI}$ & B & SE B & $\beta$ & \\
\hline Constant & 62.27 & 6.45 & & $49.54-75.00$ & 52.99 & 7.05 & & $39.08-66.89$ & 48.53 & 7.26 & & $34.21-62.86$ \\
\hline Year & 1.94 & 0.45 & $0.32 * *$ & $1.05-2.84$ & 1.81 & 0.45 & $0.30 * *$ & 0.93-2.70 & 1.68 & 0.45 & $0.28 * *$ & $0.80-2.56$ \\
\hline Age & 0.30 & 0.22 & 0.10 & $-0.13-0.72$ & 0.30 & 0.21 & 0.11 & $-0.11-0.72$ & 0.29 & 0.21 & 0.10 & $-0.12-0.70$ \\
\hline Gender & 2.60 & 1.54 & 0.11 & $-0.44-5.65$ & 2.80 & 1.51 & 0.12 & $-0.18-5.79$ & 3.46 & 1.53 & $0.15^{*}$ & $0.44-6.47$ \\
\hline Mindfulness & & & & & 0.16 & 0.05 & $0.20 * *$ & $0.05-0.27$ & 0.11 & 0.06 & 0.13 & $-0.01-0.22$ \\
\hline Self-Compassion & & & & & & & & & 0.18 & 0.08 & $0.16^{*}$ & $0.02-0.34$ \\
\hline$R^{2}$ & 0.15 & & & & 0.19 & & & & 0.21 & & & \\
\hline$F$ & $11.03 * *$ & & & & $10.81 * *$ & & & & $9.81 * *$ & & & \\
\hline$\Delta R^{2}$ & 0.15 & & & & 0.04 & & & & 0.02 & & & \\
\hline$\Delta F$ & $11.03 * *$ & & & & $8.85 * *$ & & & & $4.91 *$ & & & \\
\hline
\end{tabular}

Note. $N=192 . * * p<.01, * p<.05$

Table 9

Self-Compassion as a Mediating Variable between Mindfulness and Counselor Self-Efficacy

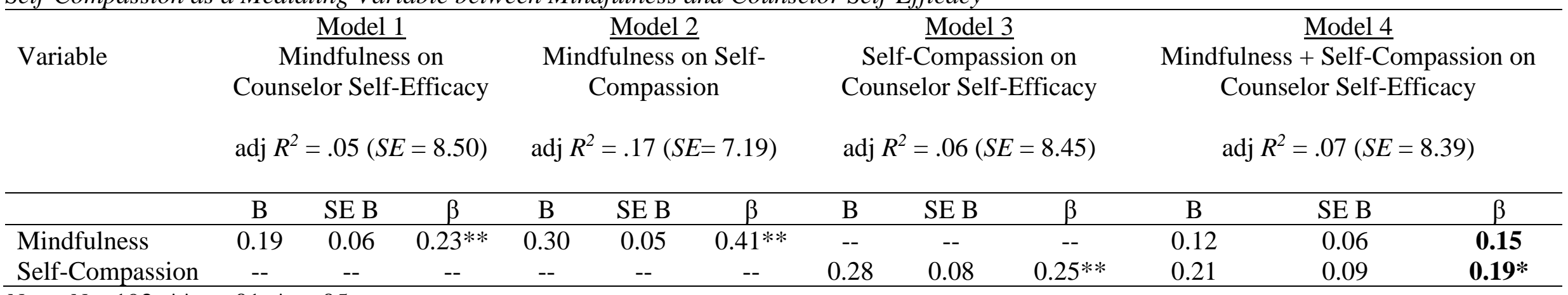

Note. $N=192 . * * p<.01,{ }^{*} p<.05$ 
The results of this analysis indicated self-compassion as a mediator variable between mindfulness and counselor self-efficacy. Table 9 shows the results of the Baron and Kenny (1986) test. Figure 2 illustrates the mediating relationships of these variables.

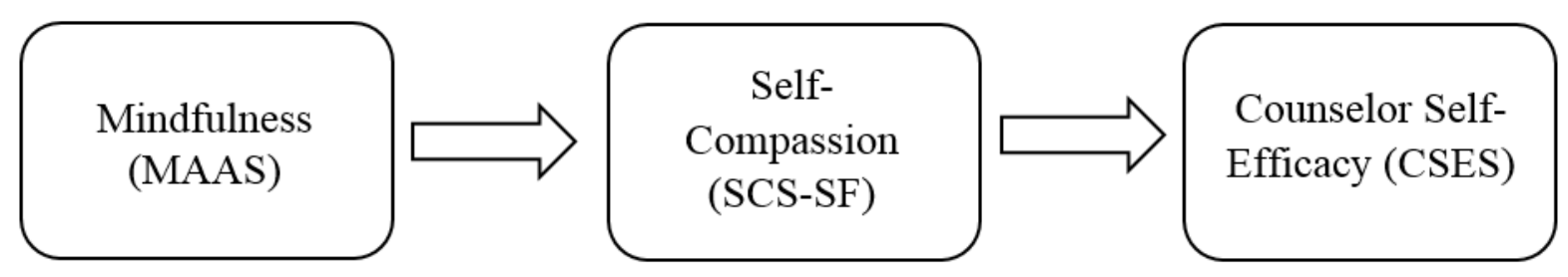

Figure 2. Visual representation of the Baron and Kenny (1986) analysis illustrating selfcompassion (SCS-SF) as a mediator variable between mindfulness (MAAS) and counselor selfefficacy (CSES).

\section{Hypotheses 4 and 5}

Hypotheses four and five stated that mindfulness and self-compassion will independently predict self-assessed professional competency while controlling for one another within regression models. To test these hypotheses, a stepwise linear regression was conducted to determine the significance of prediction among the variables. Model 1 of the first regression included covariates (year in program, age and gender) as predictor variables and average scores of self-assessed professional competency, as measured by the PCS-R, as the outcome variable. Model 2 included covariates and average scores of mindfulness, as measured by the MAAS, as predictor variables, and average scores of self-assessed professional competency as the outcome variable. Model 3 included the covariate, average scores of mindfulness, and average scores of self-compassion, as measure by the SCS-SF, as predictor variables and average scores of selfassessed professional competency as the outcome variable.

Results indicated statistical significance of the full model of covariate variables, mindfulness, and self-compassion to predict self-assessed professional competency. Average scores of self-compassion accounted for $16.4 \%$ of the variance in self-assessed professional 
Table 10

Stepwise Linear Regression Predicting Professional Competency from Covariates, Mindfulness and Self-Compassion

\begin{tabular}{|c|c|c|c|c|c|c|c|c|c|c|c|c|}
\hline \multirow[b]{3}{*}{ Variable } & \multicolumn{12}{|c|}{ Professional Competency } \\
\hline & \multicolumn{3}{|c|}{ Model 1} & \multicolumn{4}{|c|}{ Model 2} & \multicolumn{5}{|c|}{ Model 3} \\
\hline & $\mathrm{B}$ & SE B & $\beta$ & $95 \% \mathrm{CI}$ & $\mathrm{B}$ & SE B & $\beta$ & $95 \% \mathrm{CI}$ & $\mathrm{B}$ & SE B & $\beta$ & $95 \% \mathrm{CI}$ \\
\hline Constant & 57.99 & 4.69 & & $48.73-67.25$ & 51.20 & 5.13 & & $41.09-61.31$ & 45.69 & 5.15 & & $35.53-55.87$ \\
\hline Year & 0.69 & 0.33 & $0.16 *$ & $0.04-1.34$ & 0.59 & 0.33 & 0.14 & $-0.05-1.24$ & 0.43 & 0.32 & 0.10 & $-0.20-1.05$ \\
\hline Gender & 2.36 & 1.12 & $0.15 *$ & $0.14-4.57$ & 2.50 & 1.10 & $0.16^{*}$ & $0.33-4.67$ & 3.31 & 1.08 & $0.21 * *$ & $1.17-5.45$ \\
\hline Age & 0.07 & 0.16 & 0.04 & $-0.24-0.38$ & 0.07 & 0.15 & 0.04 & $-0.23-0.38$ & 0.06 & 0.15 & 0.03 & $-0.23-0.35$ \\
\hline Mindfulness & & & & & 0.12 & 0.04 & $0.21 * *$ & $0.04-0.20$ & 0.05 & 0.04 & 0.10 & $-0.03-0.14$ \\
\hline Self-Compassion & & & & & & & & & 0.22 & 0.06 & $0.29 * *$ & $0.11-0.34$ \\
\hline$R^{2}$ & 0.05 & & & & 0.10 & & & & 0.16 & & & \\
\hline$F$ & $3.51 *$ & & & & $4.98 * *$ & & & & $7.27 * *$ & & & \\
\hline$\Delta R^{2}$ & 0.05 & & & & 0.04 & & & & 0.07 & & & \\
\hline$\Delta F$ & $3.51 *$ & & & & $8.95 * *$ & & & & $14.96 * *$ & & & \\
\hline
\end{tabular}

Note. $N=192 . * * p<.01, * p<.05$

Table 11

Self-Compassion as a Mediating Variable between Mindfulness and Professional Competency

\begin{tabular}{|c|c|c|c|c|c|c|c|c|c|c|c|c|}
\hline \multirow{3}{*}{ Variable } & \multicolumn{3}{|c|}{$\underline{\text { Model } 1}$} & \multicolumn{3}{|c|}{$\underline{\text { Model } 2}$} & \multicolumn{3}{|c|}{$\underline{\text { Model } 3}$} & \multicolumn{3}{|c|}{$\underline{\text { Model } 4}$} \\
\hline & \multicolumn{3}{|c|}{$\begin{array}{c}\text { Mindfulness on } \\
\text { Professional } \\
\text { Competency } \\
\text { adj } R^{2}=.04(S E=5.88)\end{array}$} & \multicolumn{3}{|c|}{$\begin{array}{l}\text { Mindfulness on Self- } \\
\text { Compassion } \\
\text { adj } R^{2}=.17(S E=7.19)\end{array}$} & \multicolumn{3}{|c|}{$\begin{array}{c}\text { Self-Compassion on } \\
\text { Professional Competency } \\
\text { adj } R^{2}=.10(S E=5.72)\end{array}$} & \multicolumn{3}{|c|}{$\begin{array}{c}\text { Mindfulness + Self-Compassion on } \\
\text { Professional Competency } \\
\text { adj } R^{2}=.10(S E=5.70)\end{array}$} \\
\hline & $\mathrm{B}$ & SE B & $\beta$ & $\mathrm{B}$ & SE B & $\beta$ & $\mathrm{B}$ & SE B & $\beta$ & $\mathrm{B}$ & SE B & $\beta$ \\
\hline Mindfulness & 0.12 & 0.04 & $0.22 * *$ & 0.30 & 0.05 & $0.41 * *$ & -- & -- & -- & 0.06 & 0.04 & 0.11 \\
\hline Self-Compassion & -- & -- & -- & -- & -- & -- & 0.24 & 0.05 & $0.31 * *$ & 0.21 & 0.06 & $0.27 * *$ \\
\hline
\end{tabular}

Note. $N=192 . * * p<.01$. 
competency with adjusted $R^{2}=14.1 \%$. Average scores of mindfulness accounted for $9.6 \%$ of the variance in self-assessed professional competency with adjusted $R^{2}=7.7 \%$. The differences in $R^{2}$ for the models $\left(f^{2}=0.19\right)$ indicated a medium effect size according to Cohen (1988). The addition of self-compassion to the prediction of self-assessed professional competency (Model 3) led to a statistically significant increase in $R^{2}$ of .07 . In Model 3, the significance of mindfulness predicting self-assessed professional competency disappears. These findings indicated that scores on the SCS-SF were positively predictive of scores on the PCS-R above and beyond mindfulness and covariates. These findings also suggest that scores on the MAAS were not predictive of scores on the PCS-R above and beyond other variables. See Table 10 for full details on each regression model.

Similar to hypotheses three and four, the coefficient table for this analysis reflects a mediation hypothesis. To test this assumption, a Baron and Kenny (1986) test was conducted. The results of this analysis indicated self-compassion as a mediator variable between mindfulness and self-assessed professional competency. Table 11 reports the findings of the Baron and Kenny (1986) analysis. Figure 3 illustrates the mediation relationship.

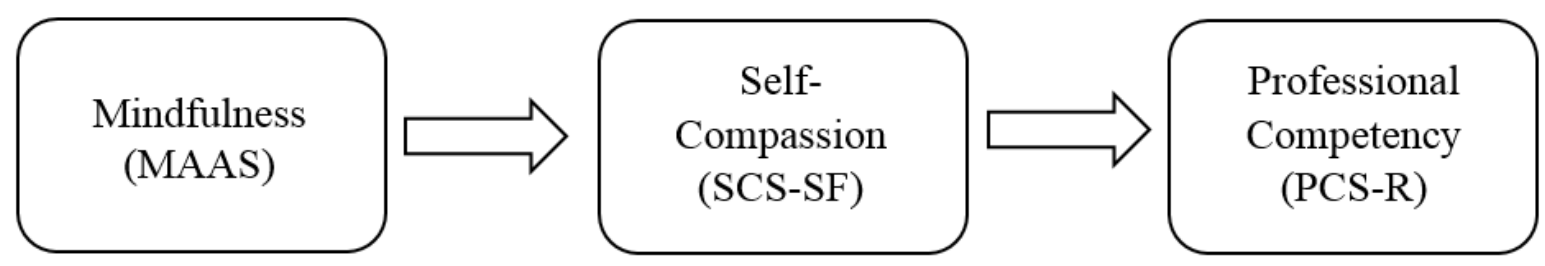

Figure 3. Visual representation of the Baron and Kenny (1986) analysis illustrating selfcompassion (SCS-SF) as a mediator variable between mindfulness (MAAS) and professional competency (PCS-R).

\section{Hypotheses 6 and 7}

Hypotheses six and seven state that training level will predict mindfulness and selfcompassion levels independently. To test these hypotheses, two stepwise linear regressions were conducted to determine the significance of prediction among the variables. Model 1 of the first 
regression included gender and age as covariates and average scores of mindfulness, as measured by the MAAS, as the outcome variable. Model 2 included covariates and average scores of selfcompassion, as measured by the SCS-SF, as predictor variables, and average scores of mindfulness as the outcome variable. Model 3 included covariates, average scores of selfcompassion and the year in program as predictor variables, and average scores of mindfulness as the outcome variable.

The results indicated statistical significance of the full model of covariates, selfcompassion and year in program as predictors of mindfulness. Year in program accounted for $17.2 \%$ of the variance in mindfulness with adjusted $R^{2}=15.4 \%$. The differences in $R^{2}$ for the models $\left(f^{2}=0.20\right)$ indicated a medium effect size according to Cohen (1988). The addition of year in program to predict mindfulness (Model 3) did not reflect a statistically significant change in $R^{2}(p=0.64)$. These findings indicated that training level was not predictive of scores on the MAAS above and beyond self-compassion and covariates. Table 12 presents the full details on each regression model.

Model 1 of the second regression included gender and age as covariates and average scores of self-compassion, as measured by the SCS-SF, as the outcome variable. Model 2 included covariates and average scores of mindfulness, as measured by the MAAS, as predictor variables, and average scores of self-compassion as the outcome variable. Model 3 included covariates, average scores of mindfulness and the year in program as predictor variables, and average scores of self-compassion as the outcome variable.

The results indicated statistical significance of the full model of mindfulness and year in program as predictors of self-compassion. Year in program accounted for $22 \%$ of the variance in 
Table 12

Stepwise Linear Regression Predicting Mindfulness from Self-Compassion and Training Year

\begin{tabular}{|c|c|c|c|c|c|c|c|c|c|c|c|c|}
\hline \multirow[b]{3}{*}{ Variable } & \multicolumn{12}{|c|}{ Mindfulness } \\
\hline & \multicolumn{3}{|c|}{ Model 1} & \multicolumn{4}{|c|}{ Model 2} & \multicolumn{5}{|c|}{ Model 3} \\
\hline & B & SE B & $\beta$ & $95 \% \mathrm{CI}$ & B & SE B & $\beta$ & $95 \% \mathrm{CI}$ & $\mathrm{B}$ & SE B & $\beta$ & $95 \% \mathrm{CI}$ \\
\hline Constant & 56.01 & 8.45 & & $39.35-72.68$ & 33.76 & 8.53 & & $16.93-50.58$ & 34.58 & 8.72 & & $17.37-57.78$ \\
\hline Gender & -1.16 & 2.04 & -0.04 & $-5.19-2.87$ & 1.03 & 1.90 & 0.04 & $-2.72-4.78$ & 0.98 & 1.91 & 0.04 & $-2.79-4.75$ \\
\hline Age & 0.14 & 0.26 & 0.04 & $-0.37-0.64$ & $>0.01$ & 0.24 & $>0.01$ & $-0.47-0.47$ & -0.05 & 0.26 & -0.02 & $-0.57-0.46$ \\
\hline Self-Compassion & & & & & 0.12 & 0.04 & $0.21 * *$ & $0.04-0.20$ & 0.56 & 0.09 & $0.41 * *$ & $0.38-0.75$ \\
\hline Year & & & & & & & & & 0.27 & 0.56 & 0.04 & $-0.84-1.37$ \\
\hline$R^{2}$ & $>0.01$ & & & & 0.17 & & & & 0.17 & & & \\
\hline$F$ & 0.35 & & & & $12.93 * *$ & & & & $9.71 * *$ & & & \\
\hline$\Delta R^{2}$ & $>0.01$ & & & & 0.17 & & & & $>0.01$ & & & \\
\hline$\Delta F$ & 0.35 & & & & $37.96 * *$ & & & & 0.23 & & & \\
\hline
\end{tabular}

Note. $N=192 . * * p<.01$.

Table 13

Stepwise Linear Regression Predicting Self-Compassion from Covariates, Mindfulness and Training Year

\begin{tabular}{|c|c|c|c|c|c|c|c|c|c|c|c|c|}
\hline \multirow[b]{3}{*}{ Variable } & \multicolumn{12}{|c|}{ Self-Compassion } \\
\hline & \multicolumn{3}{|c|}{ Model 1} & \multicolumn{4}{|c|}{ Model 2} & \multicolumn{5}{|c|}{ Model 3} \\
\hline & B & SE B & $\beta$ & $95 \%$ CI & B & SE B & $\beta$ & $95 \% \mathrm{CI}$ & $\mathrm{B}$ & SE B & $\beta$ & $95 \%$ CI \\
\hline Constant & 39.12 & 6.09 & & $27.12-51.13$ & 22.58 & 6.18 & & $10.39-34.77$ & 24.57 & 6.23 & & $12.27-36.86$ \\
\hline Gender & -3.86 & 1.47 & $-0.19 * *$ & $-6.76-(-0.96)$ & -3.52 & 1.35 & $-0.17 *$ & $-6.18-(-0.86)$ & -3.60 & 1.34 & $-0.18 * *$ & $-6.24-(-0.96)$ \\
\hline Age & 0.24 & 0.19 & 0.09 & $-0.13-0.60$ & 0.20 & 0.17 & 0.08 & $-0.14-0.53$ & 0.05 & 0.19 & 0.02 & $-0.32-0.42$ \\
\hline Mindfulness & & & & & 0.30 & 0.05 & $0.40 * *$ & $0.20-0.39$ & 0.29 & 0.05 & $0.39 * *$ & $0.19-0.38$ \\
\hline Year & & & & & & & & & 0.73 & 0.40 & 0.13 & $-0.05-1.51$ \\
\hline$R^{2}$ & 0.05 & & & & 0.21 & & & & 0.22 & & & \\
\hline$F$ & $4.79 * *$ & & & & $16.47 * *$ & & & & $13.38 * *$ & & & \\
\hline$\Delta R^{2}$ & 0.05 & & & & 0.16 & & & & 0.01 & & & \\
\hline$\Delta F$ & $4.79 * *$ & & & & $37.96 * *$ & & & & 3.45 & & & \\
\hline
\end{tabular}

Note. $N=196 . * * p<.01, * p<.05$. 
self-compassion with adjusted $R^{2}=20.6 \%$. The differences in $R^{2}$ for the models $\left(f^{2}=0.28\right)$ indicated a medium effect size according to Cohen (1988). The addition of mindfulness to predict self-compassion (Model 2) led to a statistically significant increase in $R^{2}$ of .16. The addition of year in program to predict self-compassion (Model 3) did not result in a statistically significant increase in $R^{2}(p=0.07)$. These findings indicated that year in program was not predictive of scores on the SCS-SF. See Table 13 for full details on each regression model.

\section{Summary}

In summary, the results of the study varied and three of the hypotheses were found statistically significant after controlling for the covariates and conducting stepwise linear regressions. Participants' responses indicated positive associations between mindfulness and self-compassion, self-compassion and counselor self-efficacy, and self-compassion and selfassessed professional competency. Interestingly, mediation effects of self-compassion were found throughout the analysis that may have helped to explain why mindfulness was not found to have a significant relationship with self-assessed professional competency or counselor selfefficacy independently. The next chapter will provide a summary of the findings, highlight implications for training and education programs, and discuss the strengths and limitations of the study.

\section{Summary of Findings}

\section{CHAPTER 4: DISCUSSION}

The current study explored the relationships among mindfulness, self-compassion, counselor self-efficacy and self-assessed professional competency in a sample of counseling and clinical psychology doctoral students enrolled in APA-accredited programs. The results of the study indicated statistically significant results for three of the seven hypotheses. More specifically, findings indicated associations between mindfulness and self-compassion, self- 
compassion and counselor self-efficacy, and self-compassion and self-assessed professional competency. The associations between mindfulness and self-assessed professional competency, mindfulness and counselor self-efficacy, year in program and self-compassion, and year in program mindfulness were not found to be statistically significant. This chapter will discuss the results of each analysis, possible explanations for the findings, implications for training, strengths and limitations of the study, and future directions of research in this area.

\section{Hypotheses One Through Five}

First, participant responses indicated a positive significant relationship between mindfulness and self-compassion, suggesting that greater levels of mindfulness are associated with greater levels of self-compassion in doctoral-level counseling and clinical psychology students. This finding is consistent with the vast literature exploring the relationship between these two variables (Baer et al., 2012; Baer et al., 2006; Fulton, 2016; Fulton \& Cashwell, 2015; Kabat-Zinn, 2003; Neff, 2003b).

Though the relationship between mindfulness and self-compassion was significant in this study, the results indicated different outcomes across the hypotheses suggesting that mindfulness and self-compassion may be more distinct constructs than what is conveyed through correlational analyses. This is not surprising given that the literature indicates a similar, yet contrasting, association between mindfulness and self-compassion (e.g., Germer, 2009; Neff \& Dahm, 2015). For instance, mindfulness has been defined as a cognitive process and includes regulation of one's attention to the present moment, being open to one's approach to their experience (Bishop et al., 2004), and processing information received from the environment (Langer, 1989). On the other hand, self-compassion is defined as a balance of cognitive and affective processes where individuals express kindness and understanding to themselves (Neff 2003b; Neff, 2016). Self- 
compassion as a whole is a broader experience than mindfulness, where individuals evaluate their experiences nonjudgmentally and view themselves through a lens perceiving their experiences as shared with humanity (Neff, 2016). For these reasons and to better understand mindfulness and self-compassion as independent and perhaps complimentary constructs, each variable was controlled for in relation to the other throughout the analyses.

Self-compassion was shown to have a positive significant relationship to counselor selfefficacy. By identifying the components of counselor self-efficacy, readers can better understand the relationship among these variables. Counselor self-efficacy is defined as a belief or judgment about one's ability to work effectively with clients (Larson \& Daniels, 1998) and is focused on specific skillsets and behaviors that are present during session (Constantine \& Ladany, 2001). Through this definitional understanding, it is apparent why self-compassion may be related to counselor self-efficacy. The emphasis that counselor self-efficacy has on evaluation and judgement about one's performance relates to all three aspects of self-compassion (e.g., kindness, mindfulness and common humanity). This becomes clearer when considering the foundation of self-compassion, specifically its development and growth in the self-evaluation literature. The evaluative component of self-efficacy is elicited through both the cognitive and affective aspects that are brought about when practicing self-compassion.

Participant responses indicated a positive statistically significant relationship between self-compassion and self-assessed professional competency, such that greater levels of selfcompassion are associated with higher levels of self-assessed professional competency. Expanding on Kaslow et al.'s (2004) definition of competency, self-assessed professional competency is defined as a clinician's assessment of their attitudes, beliefs, knowledge and skills in working with clients. Thus, in order for a clinician to self-evaluate, they are required to have 
an understanding of their skill level, engage in self-reflective practices, and have awareness of their attitudes and their impacts on various dimensions of training. Given this definition, it makes sense that self-compassion is significantly associated with self-assessed perceived competency. Self-compassion focuses on an overarching view of self, which is necessary to engage in accurate self-evaluation. Additionally, self-compassion and self-assessed perceived competency share affective components, which extends beyond the cognitive components that underlie mindfulness and counselor self-efficacy. This finding indicates that self-compassion may be more associated with generalized views of and feelings toward oneself. Further, Neff (2003b) reported theoretical associations between self-compassion and insight into one's limitations of their performance while noting that empirical findings of this relationship are nonexistent in the literature. Thus, the findings in the present study provide empirical evidence to fill the gap proposed by Neff (2003b) in the literature on this topic. This finding also illuminates the relationship self-compassion has with self-evaluative processes, which has various implications for doctoral-level psychology training and education programs.

On the other hand, mindfulness was not found to be statistically significantly associated with self-assessed professional competency or counselor self-efficacy. These findings conflict with the results of past literature that suggests a significant relationship between mindfulness and counseling skill development (Buser et al., 2012; Ying, 2009) and multicultural competence (Ivers et al., 2016). There are two proposed reasons for these discrepancies in the literature. First, it is important to consider how competency was measured in previous studies. Researchers used the Counseling Skills Scale (Eriksen \& McAuliffe, 2003) and the Multicultural Counseling Competence and Training Survey-Revised (Holcomb-McCoy \& Myers, 1999), neither of which are framed in a way to represent or reflect the current APA benchmarks of competency in 
doctoral trainees. Though the findings from previous studies suggest that mindfulness practice can be a foundation of enhanced counselor performance and professional competency, this assumption cannot be validated without appropriately measuring performance based on the standards of competency that are set forth in accredited training programs. The release of the validated PCS-R (Taylor, 2015), which was used in the present study to measure self-assessed professional competency, has provided an opportunity for researchers to more accurately assess competency foundationally based on the benchmark system introduced by the APA.

Second, it may be that mindfulness was directly significantly related to the outcome variables because of the potential mediation effect of self-compassion. In both analyses where mindfulness was the predictor variable, the significance of the findings was present until the variable of self-compassion was added as a covariate. After completing two Baron and Kenny (1986) tests for these analyses, it became evident that self-compassion was indeed a mediator variable in both relationships between mindfulness and counselor self-efficacy and mindfulness and self-assessed professional competency. These findings are consistent with previous literature which illustrated the mediating effects that self-compassion has in the relationship between mindfulness and other variables. For instance, researchers have found that self-compassion mediated the relationship between mindfulness and emotional well-being (Bluth \& Blanton, 2014), mindfulness meditation and psychological well-being (Baer et al., 2012), and mindfulness and stress reduction (Birnie et al., 2009). Overall, the results of the present study indicate that mindfulness is important in the development of counselor self-efficacy and self-assessed professional competency; however, the relationship can only be explained when considering selfcompassion as a mediator within these relationships.

\section{Hypotheses Six and Seven}


Participants' year in their doctoral program was not shown to be positively related to levels of self-compassion or mindfulness. This finding suggests that as doctoral students advance through their applied psychology program, their levels of self-compassion and mindfulness vary and are not linearly significant. There are two potential reasons for these outcomes. First, there are several variations between and within clinical and educational training in counseling and clinical psychology programs that make it difficult to track consistencies over time. For example, programs differ in their course schedule and their requirements for clinical training. Sipps et al. (1988) concluded that variations in trainees' personal and professional attributes differed based on the course of academic and applied training rather than solely focusing on differences across progression through the academic program. These program differences may have been reflected in the results of the current study in that trainees' experiences vary based on their program structure even when they identify a similar training year as other participants. The finding in the present study cannot be fully understood without considering variations in education and training programs in counseling and clinical psychology.

Second, when reviewing the data through bar graphs, it becomes evident why both mindfulness and self-compassion do not exhibit a linear trend across training year with this sample. It appears that both mindfulness and self-compassion scores increase linearly during the beginning years of training and then vary across participants after the $4^{\text {th }}$ year. More specifically, the findings of this study show that participants' level of mindfulness increase from $1^{\text {st }}$ year to $4^{\text {th }}$ year, with a slight decline in average scores during the $5^{\text {th }}$ year before increasing again in the $6^{\text {th }}$ year. During the $7^{\text {th }}$ year, it appears that participants' scores of self-compassion fall to their lowest average across all years. The trends of self-compassion show a similar trend where 
average scores increase until the $6^{\text {th }}$ year where the scores slightly decrease before a large decrease in scores during the $7^{\text {th }}$ year. See Figures 4 and 5 for bar graph illustrations.

Figure 4. Bar graph illustrating mean scores of mindfulness, as measured by the MAAS, across training year.

This visual representation through inspection of bar graphs indicates large difference among training years. However, it is important to consider the sample of the study when interpreting these results. Majority of the participants $(84.5 \%)$ in this study indicated being between their $2^{\text {nd }}$ and $5^{\text {th }}$ year in the training program. Only 30 participants $(15.5 \%)$ endorsed

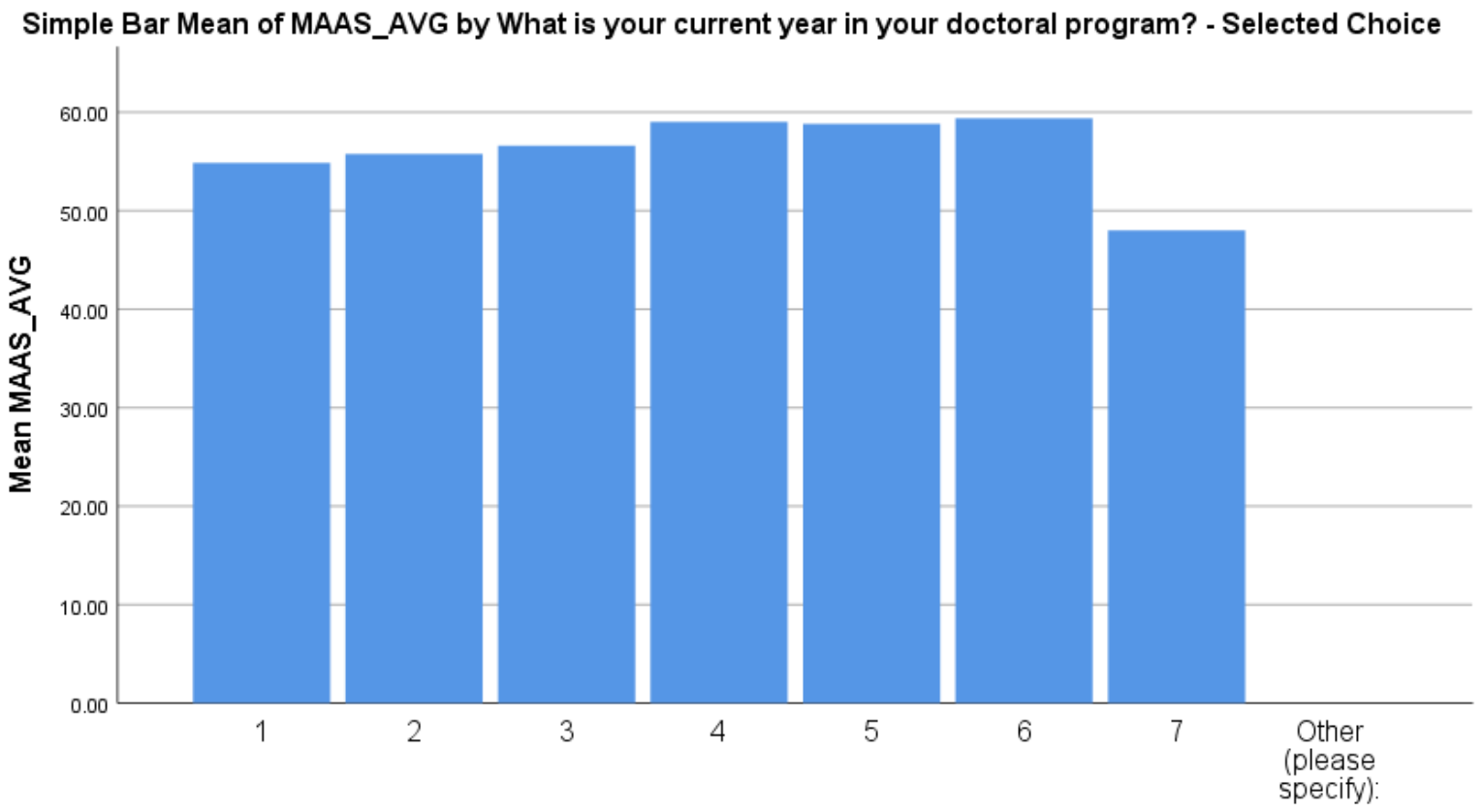

What is your current year in your doctoral program? - Selected Choice

being either in the first year of their program or beyond their $5^{\text {th }}$ year. More specifically, only 2 participants $(1 \%)$ identified being enrolled in the $7^{\text {th }}$ year of their program. Thus, the variance in the sample may reflect the variance in the average scores of mindfulness and self-compassion across training year illustrated in Figures 4 and 5. 


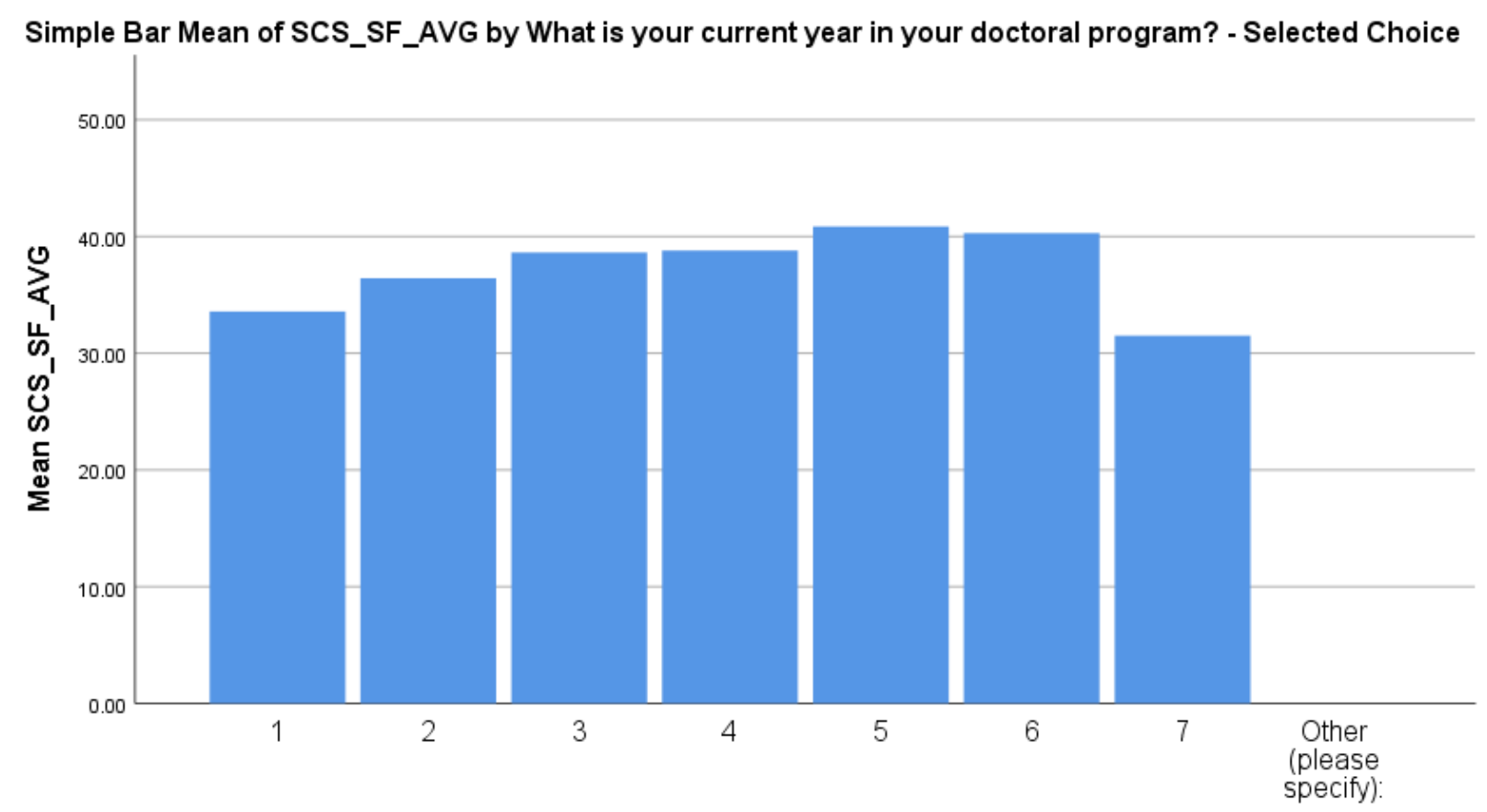

What is your current year in your doctoral program? - Selected Choice

Figure 5. Bar graph illustrating mean scores of self-compassion, as measured by the SCS-SF, across training year.

\section{Implications for Training}

\section{Suggestions for Evaluation}

There are several potential implications for doctoral-level psychology education and training programs based on the results of this study. On a broad level, one of the most important implications is to consider ways that trainees are evaluated. Roberts and colleagues (2005) emphasized a current culture shift toward career-long assessment of competence, and argued that trainees and practicing professionals need to document self-assessment practices and competence-building activities that they are engaged in. First and foremost, it is important that supervisors and faculty advisors use the APA (2011) Revised Competency Benchmarks as the foundational outline for evaluation. Above and beyond supervisors' use of this benchmark system, it is critical that trainees are offered an opportunity to evaluate themselves to measure their levels of self-efficacy and perceived competency. For years, researchers have argued that 
self-efficacy should be considered in relation to performance capabilities and competencies for trainees (e.g., Constantine, 2002; Larson \& Daniels, 1998; Lent et al., 1998); however, most literature that examines competency-based variables in trainees only focuses on supervisor responses and perspectives. As a way to gather more holistic data about trainee's experiences and competencies, supervisors and educators should integrate trainees' self-evaluation alongside their self-appraisal of counselor self-efficacy.

Training programs can implement self-assessment in various ways. For example, Kozina et al. (2010) highlighted the importance of trainees completing self-assessments of their competence throughout supervision. These self-assessments can be used effectively in supervision to identify strengths, areas of growth, and direction of supervision given the context of the self-assessment. In educational settings, faculty advisors can engage trainees in selfevaluation using the benchmark standards where trainees and advisors independently evaluate the trainee before meeting together to discuss similarities and differences in perceptions and outcomes (e.g., Greason \& Cashwell, 2009). Trainees' counselor self-efficacy can also be regularly monitored and evaluated by both the trainee and their supervisor through use of appropriate instruments, such as the CSES (Melchert et al.,1996), COSE (Larson et al., 1992), and CASES (Lent et al., 2003). By engaging both the trainee and the evaluator in this process, trainees' self-efficacy can enhance and become more accurately reflective of their skillset and competencies.

Above and beyond identifying implications for accurate assessment of trainees, the present study focused on ways to enhance counselor self-efficacy and self-assessed perceived competency of doctoral-level counseling and clinical psychology trainees through examination of mindfulness and self-compassion variables. Data from the current study illustrated the 
predictive effect that self-compassion has on counselor self-efficacy and self-assessed perceived competency. In addition, the results indicated a potential mediating effect that self-compassion has on the relationship between mindfulness and self-assessed professional competency and counselor self-efficacy. Though mindfulness and self-compassion have been an area of focus in mental health fields, the area of attention has primarily been dedicated to research and utilization as clinical interventions with little focus on implications for non-clinical populations.

Understanding that mindfulness and self-compassion practices impact trainees' perceptions of their abilities, future research should explore and examine ways to adapt evidence-based mindfulness and self-compassion practices for use with a trainee population. As a whole, it is critical that training programs, both educational and practice-based, help trainees develop a sense of self-efficacy and perceived competence in their ability to perform the work that is expected of them through mindfulness and self-compassion practices. The following sections identify suggestions for programs to include mindfulness and self-compassion activities and experiential exercises for their students.

\section{Informal Mindfulness and Self-Compassion Practices}

Informal practices of mindfulness and self-compassion can vary in creativity and implementation and can be individualized. The intention behind informal practices entails that trainees engage in mindfulness and self-compassion practices outside of a formal setting and/or practices that do not involve teaching or specific guidance. For instance, participants in the current study indicated engagement in the following practices: self-compassion breaks, relaxation exercises, mindful walking, yoga, journaling and writing, physical exercise, mindful cooking and eating, prayer, listening to music, art, engagement in personal therapy, utilizing mindfulness and self-compassion in clinical practice, martial arts, crocheting, and massage. It is 
important to note that the distinction between engaging in many of the above practices on a daily basis regularly and engaging in with the intention of practicing mindfulness and self-compassion is based on the purpose; if trainees engage in these behaviors with the intent to practice and purpose of practicing mindfulness and self-compassion as it relates to their personal and professional growth, the practice itself may be considered informal practice.

Examples of mindfulness and self-compassion informal practices in educational settings may include assignments and experiential activities that foster personal and professional growth through self-reflection. Assignments that require trainees to introspect and examine their strengths and growth areas can be considered practices with the intent to enhance mindfulness and self-compassion (e.g., Goreczny et al. 2015). In fact, Ivers and colleagues (2016) found that trainees who were given the opportunity to both process their internal and external stimuli and express their thoughts and feelings about their experiences were more effective in working with diverse clientele, which extends to achieving greater levels of cultural competency in counseling. Thus, instructors should consider ways to implement reflective practices in their course structure and assignments when teaching doctoral-level psychology trainees as a way to foster selfefficacy and competency.

In supervisory contexts, trainees can learn and engage in mindfulness and selfcompassion practices in various informal ways. First, supervisors can model techniques to enhance mindfulness and self-compassion in their supervisees through various theoretical lenses and supervisory orientations. Supervisors can also encourage trainees' skill-building processes using mindfulness and self-compassion as a foundation of their approach, such as encouraging role play practices with the focus on observing without judgment. Lent and colleagues (1998) outlined examples of prompting questions that supervisors can ask of their trainees in helping 
them to prepare for upcoming sessions. These types of prompts can be adapted to fit the supervisory approach, case conceptualization, supervisory goal-setting, client goal-setting, clinical skill utilization, or any other important aspect that is deemed appropriate by the supervisor.

Overall, informal practices of mindfulness and self-compassion are broader than those that are considered formal because they have less evidence basis. However, these practices are no less important than those that are formal. Instead, informal practices should complement and supplement formal practices that are implemented in educational and training settings for trainees as a way to instill these values so that they become habitual rather than task oriented. Though the present study collected information regarding participants' involvement in mindfulness and self-compassion exercises, it was out of the scope of this study to examine specific associations between engagement in practices and effects on counselor self-efficacy and self-assessed perceived competency. Future research should explore specific practices of mindfulness and self-compassion to better understand the implications of engaging in informal practices.

\section{Formal Mindfulness and Self-Compassion Practices}

Mindfulness and self-compassion can be practiced in both formal and informal ways, and educational and training settings could benefit from implementing either or both types of practices in their curriculum and structure. In some ways, training programs may already be implementing these practices, as evidenced by Fulton and Cashwell's (2015) study which found that $37 \%$ of their sample of master's level trainees indicated receiving mindfulness education and/or experience from their program of training. The present study collected information from participants regarding their informal practices of mindfulness and self-compassion, though these 
variables were not considered within the analysis. It is difficult to make assumptions of these outcomes based on the lack of literature that exists that explores informal mindfulness and selfcompassion practices. Therefore, it is important to consider more formal means of practice to develop examples of how mindfulness and self-compassion practices can be implemented within existing educational and training structures.

Majority of the literature that examines mindfulness and self-compassion practices include more formalized programs. Several programs and protocols have been developed for use with diverse populations with the intent to enhance these practices in participants. Programs such as mindfulness-based stress reduction (MBSR), mindfulness-based role playing (MBRP), mindful self-compassion (MSC), self-compassion online (SCO), and loving-kindness meditation (LKM) have been heavily researched within the field mindfulness and self-compassion literature and have illustrated effective results with participants, as follows.

Mindfulness-Based Stress Reduction (MBSR; Kabat-Zinn, 1990). Mindfulness-based stress reduction (MBSR) is a structured group program of mindfulness training that was originally developed by Kabat-Zinn (1990). This program consists of both formal (e.g., breathfocused attention, body scan-based attention to sensory experiences, shifting attention, monitoring moment-to-moment experiences, walking meditation, and eating meditation) and informal (e.g., pausing, shifting attention to present moment) forms of mindfulness practice and hatha yoga. The program spans 8 weeks and consists of two-and-a-half hour long classes with one "day of silence" in between the $6^{\text {th }}$ and $7^{\text {th }}$ weeks (Kabat-Zinn, 1990).

A meta-analysis found that MBSR used with healthcare professionals (e.g., physicians, psychologists, nurses, social works, physical therapists, medical students, counseling students) resulted in increased empathy and self-compassion, and reduced anxiety, depression, burnout, 
perceived stress, and rumination (Irving et al., 2009). Additionally, in a study sampling clinical social work graduate students, Gockel et al. (2013) identified a significant positive relationship between engagement in a MBSR program and counseling skill development and counselor selfefficacy. Further, when asked about their experience, students indicated that MBSR training was "helpful to their learning in the classroom and facilitative of their development of counseling skills in a number of specific areas" (Gockel et al., 2013, p. 350).

With the program structure in mind, it may be more appropriate for educators to implement the MBSR program as part of their curriculum as opposed to supervisors integrating training into practicum experiences. The study conducted by Gockel and colleagues (2013) included the MBSR program as part of the curriculum for a course offered to clinical social work students who voluntarily expressed interest. This example illustrates that course curriculum and structure can be adapted to include programming to enhance trainees' mindfulness and selfcompassion. For example, MBSR can be included as a section within the curriculum of a counseling techniques course with the intent to help enhance trainees' mindfulness and selfcompassion for personal and professional development within a clinical context. Another suggestion for implementation could be to offer MBSR programming to students as part of an experiential requirement for a course where students are provided resources to be involved in the formalized program outside of the classroom. MBSR training is available online and through published manualized books. It is encouraged that researchers and educators use the manualized program to facilitate ways to adapt the mindfulness training to instructors and students (Fulton \& Cashwell, 2009). Future research should investigate the impact MBSR involvement has on graduate level trainees and further explore ways to implement MBSR programs in curriculum and training. 


\section{Mindfulness-Based Role-Play (MBRP; Andersson, Kind, \& Lalande, 2010).}

Mindfulness-based role-play is particularly used in supervision as a way to enhance clinicians' empathic understanding of clients (Andersson et al., 2010). Similar to MBSR, MBRP is a manualized protocol and involves various stages (acquainting stage, theme stage, and sharing stage). The supervisor's role in MBRP is to guide their supervisee toward dialogical mindfulness, which is the experience of being mindfully aware of their phenomenological experience in each role they participate in in the dialogue (Andersson et al., 2010). When used with various types of mental health clinicians (psychologists, counselors, psychotherapists, social workers, mental health nurses, art therapists, psychiatrists), participants endorsed enhanced awareness of their functioning, affirmation of their functioning, and awareness of their deficits as clinicians (Andersson et al., 2010).

Though the formal practice of MBRP is used with the intent to gain awareness of clients' experiences, the findings of Andersson et al.'s (2010) study suggested broader outcomes related to clinician's level of awareness, attentiveness and self-understanding. Whereas the MBSR manualized program could be more effective within teaching curriculum, MBRP could be supplemental to educational training and practiced within the applied setting of a practicum or internship site. This type of practice can influence trainees' personal and professional development as it applies to engagement in mindfulness practices and attention to clinical skill utilization and reflective practice. Considering this is a fairly new manualized protocol, future researchers should explore validation and reliability of this protocol with use in samples of counseling and clinical trainees at various stages of training.

Mindful Self-Compassion (MSC; Neff \& Germer, 2012). Mindful Self-Compassion (MSC) was developed by Neff and Germer (2012) and modeled off of MBSR as a "hybrid" 
program applicable to both the general population and clinical populations (p. 3). The program is designed to enhance participants' self-compassion and includes a secondary emphasis on mindfulness. Participants of the program meet for two-and-a-half hours once a week over the course of 8 weeks and attend a half-day meditation retreat. MSC is typically led by two facilitators in a group format and includes both formal (meditation) and informal (daily practices, interpersonal exercises) self-compassion practices. Results of the validation study indicate increased levels of mindfulness, self-compassion, other-compassion and life satisfaction, as well as reduced levels of anxiety, depression, stress and avoidance in samples of general and clinical populations (Neff \& Germer, 2012).

Following the same structure as MBSR, MSC can be implemented into the curriculum of educational training programs. Neff and Germer (2012) suggest that MSC is, in fact, a complimentary program to MBSR and can be taught simultaneously or in collaboration with one another. Because of the focus on basic principles of mindfulness and self-compassion, MSC may be more effectively used with incoming graduate students and earlier on in training; however, implementation is dependent on the understanding that mindfulness and self-compassion have already been taught and practiced throughout the program and within practicum sites. No literature exists on the use of MSC with trainee populations, which evidences a gap in the literature. Given the manualized protocols exist for each program, educators can be creative about how to structure both MSC and MBSR programming into their curriculum.

Self-Compassion-Online (SCO; Finlay-Jones et al., 2016). Self-Compassion Online (SCO) was developed by Finlay-Jones et al. (2016) for administration in an online environment and validated with a sample of psychology trainees. The theoretical basis for SCO included compassion-focused therapy and mindfulness- and acceptance-based interventions (e.g., Gilbert, 
2010; Roemer et al., 2009). SCO provides psychoeducation about self-compassion, meditation training, and reflective and experiential exercises (Finlay-Jones et al., 2016). SCO is self-paced and separated into six modules that are administered over the course of six weeks. Psychology trainees who participated in the validation study endorsed significant improvements in selfcompassion, happiness, and emotion regulation, and reduced levels of depression and perceived stress (Finlay-Jones et al., 2016). Considering this study is the only study to date that utilized SCO, various limitations of this program are notable (see Finlay-Jones et al., 2016).

With the limitations of this study in mind, SCO appears to be an appropriate program that may be more feasible to implement in training settings. Because of the online nature of SCO, instructors may be able to include $\mathrm{SCO}$ as part of recommended or required assignments within a course structure. The flexible structure of SCO may also be more feasibly implemented for trainees in both educational training and practicum settings where trainees may be able to apply these skills for personal and professional development at their own pace. Though future research is necessary to explore the validation and reliability of SCO, the original study exemplifies the creative and flexible approach to implement mindfulness and self-compassion in training programs for psychology trainees. In addition, the recency of this publication also indicates the potential trend of future literature addressing ways to integrate technology and skill-based programs for use with training populations.

Loving-Kindness Meditation (LKM; Salzberg, 1995). Loving-Kindness Meditation (LKM), also known as Metta meditation, is a practice that focuses on developing love and releasing negative emotions (Salzberg, 1995). Similar to mindfulness, the foundations of LKM are rooted in Buddhist tradition and involve variations of meditations that incorporate nonjudgmental attention to the present moment and foster feelings of kindness toward oneself 
without denying one's actual experience (Fredrickson et al., 2008; Leppma, 2012; Shahar et al., 2014). Some core principles of LKM include practicing with an attitude of curiosity; reconnecting with oneself by healing internalized thoughts, emotions, and physical sensations; and feeling interconnected with oneself and others through recognizing the "essential nature of others" as well as oneself; and owning a sense of humanity similar to mindfulness approaches (Leppma, 2012, p. 198). In a study with counseling trainees, researchers found that trainees who engaged in hour long sessions of LKM psychoeducation and practice across six weeks endorsed improvements in mood and emotional and cognitive empathy, all which indirectly impacted personal wellness and counseling efficacy (Leppma \& Young, 2016).

Group sessions that include LKM are often similarly structured as the manualized programs of MBSR, MSC and SCO. Typical LKM sessions include a form of check-in, psychoeducation, LKM exercise, processing and discussion (see Fredrickson et al., 2008; Leppma \& Young, 2016). However, the flexibility of the topic of LKM and length of meditative exercise can vary based on the population it is geared toward and the intent of the program. In general, LKM practices entail a formal meditation and other exercises that purposefully and unconditionally direct feelings of compassion, kindness, and warmth to oneself and others (Shahar et al., 2014). Similar to the structured programs mentioned above, LKM can easily be woven into course curriculum and assignments throughout training as a way to enhance self- and other-compassion in trainees. Researchers should continue exploring ways to implement LKM within course curriculum with consideration of time commitment of meditation and adaptability to trainee populations. 


\section{Strengths of the Study}

The ideological foundations of the present study indicate various strengths of the research. First, the design and aim of the study responds to several calls to action outlined in previous literature suggesting further exploration to differentiate self-efficacy and professional competency (e.g., Bong \& Skaalvik, 2003; Hughes et al., 2011; Lent et al., 1998) and mindfulness and self-compassion (e.g., Neff \& Dahm, 2015), as well as factors that impact these outcomes (e.g., Bradley et al., 2012; Roberts et al., 2005; Ruggiero et al., 2014). Through examining the similarities and differences among all four variables, the present study addresses this gap in the literature and responds to ways researchers have directed the literature in this field.

Second, the present study is one of the initial research studies using the PCS-R to measure self-assessed perceived competency in counseling and clinical doctoral trainees. This is important because the PCS-R is the most recent measure that accurately reflects components of competency that are directly associated with APA's benchmark system. The present study further validated the measure and found it to be reliable with a doctoral-level trainee sample. Further, the present study also replicated results found in previous studies using the same variables (mindfulness, self-compassion, and professional competency) to more accurately report outcomes associated with the most updated version of competency benchmarks through use of the PCS-R.

In addition, the data collected in the present study presents various strengths that are important to note. First, few studies examine these variables within a sample of counseling or clinical psychology doctoral-level trainees. Most of the literature within this field assesses aspects of mindfulness and self-compassion and how these variables may relate to self-efficacy 
and competency in high school students, clinical samples, or other healthcare provider fields, such as social workers and medical students. Thus, the present study is one of few studies that examine the association among these variables with a sample of doctoral-level counseling and clinical psychology trainees.

Third, the demographic data of the participants appears to closely represent the demographic breakdown of the population of the study. The present study sampled counseling and clinical doctoral-level students who were enrolled in an APA-accredited programs. The demographics in the present study reflected the following: $82.3 \%$ women, $70.8 \%$ White/Caucasian American, 7.8\% African American/Black, 7.3\% Hispanic/Latinx, 5.7\% Asian American/Pacific Islander, 4.2\% biracial/multiracial, 2.1\% Arabic American/Middle Eastern, and 1\% East Indian/Indian American. The most recent summary report of student demographics released by the APA (2016) indicated the following breakdown for students enrolled in all doctoral psychology-related degrees for the 2014-2015 academic year: $72.2 \%$ female, $72.4 \%$ White/Caucasian, 10.8\% Asian/Pacific Islander, 7.5\% Hispanic/Latino, 6.1\% African American/Black, $2.5 \%$ multiethnic, and 0.7\% American Indian/Alaska Native. Arabic American or Middle Eastern were not identified as ethnic categories in this report.

The breakdown of psychology programs was also reflective of the percentage differential between APA-accredited programs. The present study indicates $72.4 \%$ of participants are from clinical psychology programs. APA's (n.d.) website shows that there are 237 clinical programs (75.7\%) and 76 counseling programs $(24.3 \%)$ that are accredited. Lastly, the type of program (Ph.D. vs Psy.D.) breakdown of the study was also closely representative of APA programs in general. The present study indicated $67.7 \%$ of participants were from Ph.D. programs and 32.3\% 
from Psy.D. programs. APA's (n.d.) website indicates of all the counseling and clinical programs, $241(77 \%)$ are Ph.D. and $72(33 \%)$ are Psy.D.

\section{Limitations and Recommendations for Future Research}

\section{Research Design}

There are various limitations of the study that are important to note. The research design itself poses various threats to internal and external validity. First, the correlational research design of the study creates challenges in determining causality of the relationships that were found. Heppner et al. (2008) identified this threat to internal validity as "ambiguous temporal precedence" (p. 92) which indicates that because independent variables in this study were not manipulated, the direction of the relationship between the variables in the various hypotheses is unclear. The purpose of using a correlational design in this study was to fill in the gap in the literature to determine whether relationships exist among the variables. Considering three of the seven hypotheses were found significant, future research could better determine causality using different research designs.

Second, the self-report method used for this study creates a limitation. All variables were measured using a self-report, survey method for this study, which Heppner et al. (2008) explained could result in "mono-method bias" (p. 99). Heppner et al. (2008) stated that not only do "self-report measure[s] often share a common respondent bias" (p. 99), but they also do not allow for extensive validation and reliability of the results. Specific to the assessment of professional competency, Roberts et al. (2005) stated that not only should competency be evaluated through "measurable indicators of behaviors, skills, and attitudes and values," (p. 358) but that these indicators are more accurately evaluated when assessing multiple sources. The scope of this study specifically involved measuring self-assessed professional competency to be 
consistent with the self-assessment of the other variables (counselor self-efficacy, mindfulness, and self-compassion). However, this study design could have been strengthened by examining these variables from multiple sources, such as trainee, practicum supervisor, and academic advisor, as a way to substantiate the findings and determine more accurate representations of the relationships among these variables.

Additionally, while the measures chosen for this study were based on existing literature, it is possible that other instruments would better represent the chosen variables. Specifically, the MAAS, CSES and PCS-R all posed threats to the study. As noted above, the MAAS (Brown \& Ryan, 2003a) has been criticized in recent literature to be limited in its measure of mindfulness. The MAAS (Brown \& Ryan, 2003a) measures attention to and awareness of present moment experiences and was used in the present study to reflect distinct differences between measures of self-compassion and mindfulness. However, to obtain a more general and holistic sense of mindfulness in participants, Van Dam et al. (2018) argued that researchers use instruments such as the Freiburg Mindfulness Inventory (FMI; Walach et al., 2006) or the Five Facet Mindfulness Questionnaire (FFMQ; Baer et al., 2006). In the current study, the hypotheses where mindfulness was a predictor variable and the hypothesis where mindfulness was an outcome variable were not found to be statistically significant. Therefore, score interpretations from the MAAS were consistent across all analysis that included other variables. Considering Van Dam et al.'s (2018) article was published during the data collection phase of the present study, the researchers were unable to implement the recommendations of the researchers. Future research should consider measuring mindfulness with different instruments to consider their relationships with selfassessed perceived competency and year in training. 
The CSES (Melchert et al., 1996) was created in 1996 and measures the degree of confidence a counselor has in their counseling abilities. Several other measures of counselor selfefficacy have been published more recently, such as the Counselor Activity Self-Efficacy Scales (CASES; Lent et al., 2003), and used more frequently, such as the Counseling Self-Estimate Inventory (COSE; Larson et al., 1992). The CSES was chosen by the investigators in this study because of the focus on general self-efficacy in the field of psychology, as opposed to more novice or specified skills that may not be applicable to all participants of the study. Lastly, the PCS-R (Taylor, 2015) was chosen by the investigators for this study because it reflects the current benchmark evaluation system produced by the APA. As acknowledged above, the existing literature using this measure notes the limitations in reliability across different samples and over time. Future research should explore how utilization of different instrumentation influences the relationships among these variables.

\section{Data Analysis and Findings}

The first limitation in data analysis includes the removal of incomplete datasets. A total of 59 survey responses were listwise deleted from the dataset prior to analysis due to incomplete data, ineligible participation and unequal sample size within demographic categories. The removal of response due to ineligible participation and unequal sample size are discussed in the findings section. Regarding incomplete data, visual inspection of the dataset indicated that there was no reason to suspect any systematic pattern within these datasets. Considering the measures were counterbalanced in this study, the cause of incompletion cannot be confirmed.

Second, to further the literature future researchers should explore variations in data analysis with self-compassion, mindfulness, counselor self-efficacy and self-assessed professional competency as variables. Researchers should explore the association among these 
variables using a mediation regression to more accurately determine whether self-compassion influences the relationship between mindfulness and counselor self-efficacy and self-assessed professional competency. In addition, researchers should conduct a moderation regression to determine whether self-compassion influences the strength or direction of the relationship between mindfulness and counselor self-efficacy and self-assessed professional competency. Researchers should also conduct structural equation modeling to more accurately determine the structure and order of the relationship among these variables. Overall, considering the findings of the present study illuminated the relationship among these variables, future researchers can focus on more complex ways to analyze the data using these variables with the goal of better understanding these relationships.

In addition, considering that the SCS-SF and PCS-R include several subscales as part of the measure, it may be beneficial to assess specific components of these variables and their relation to the findings. For instance, the SCS-SF includes six subscales (self-kindness, selfjudgment, common humanity, isolation, mindfulness, and over-identified) and the PCS-R, as used for the present study, included eight subscales (professionalism, reflective practice, scientific knowledge, relationships, interdisciplinary systems, assessment, intervention, and consultation). The findings of the present study indicate a relationship between self-compassion and self-assessed professional competency and counselor self-efficacy, though the analyses did not extend beyond association of the general variables. Future literature should explore specific subscales of within these two constructs to determine the strength and direction that each component has with one another.

With regard to the findings of the study, three limitations stand out that should be considered for the future directions of the literature. First, the unequal sample size distribution 
throughout practicum/internship enrollment resulted in this demographic variable not being included as a covariate in the primary analyses. Majority of the sample $(56.2 \%)$ in the present study indicated practicing at either a university counseling center or hospital setting. The most recent release of data summaries by APPIC (2011) indicated that university counseling centers and hospital settings made up only $32 \%$ of total internship sites. Therefore, the lack of variation in sample size across the site options makes it difficult to generalize these findings to all counseling and clinical psychology trainees.

Second, the present study collected information from participants regarding their involvement in mindfulness and self-compassion practices; however, these practices were not considered for the primary analyses because of the specific scope of the study examining the relationships among self-compassion, mindfulness, counselor self-efficacy, and self-assessed professional competency. Using this demographic variable in future studies could assist in substantiating the operationalization of the variables (mindfulness and self-compassion) and could more holistically examine traits of these variables that may impact their association with outcome variables. For example, Van Dam et al. (2018) suggested a more comprehensive approach to surveying individuals' self-reported levels of mindfulness through assessing individuals' awareness of their involvement and engagement in various specific practices. Thus, by including mindfulness and self-compassion practices as a variable to be considered in the primary analysis in future literature, researchers can more comprehensively explore variations in and between these variables.

Furthermore, Neff and Dahm (2015) argued that self-compassion is a "teachable skill that is dose dependent" (p. 129), suggesting that the more an individual engages in self-compassion practices, the more they will learn these techniques and integrate them into their daily life. 
Gockel and colleagues (2013) also found that MBSR training enhanced counselor self-efficacy and participants' ability to learn and be open to clinical training exercises in the classroom. These findings were indicative of outcomes post-test as well as up to three months after completion of the training, which evidences the teachability and habitual nature of mindfulness practices. Though the results of the present study did not reflect significant relationships among training year, mindfulness and self-compassion, information about participants' engagement in mindfulness and self-compassion practices was collected. Considering this variable was not considered in the primary analyses, the relationship between training year and engagement in practices is unclear. Future research should continue to explore these variables to determine appropriate implementation of practice within educational and training programs.

Lastly, training year was used in the present study as a way to identify differences among trainees at varying levels of training. However, it is important to note the distinctions within and between training year across different training programs. For instance, APPIC reported variation among participants of the internship match process during the 2018-2019 academic year. The results of this match process indicated that majority of students were in their $4^{\text {th }}$ year or $5^{\text {th }}$ year (42\% and $35 \%$, respectively), with fewer participants endorsing being enrolled in their $3^{\text {rd }}$ year $(9 \%), 6^{\text {th }}$ year $(10 \%), 7^{\text {th }}$ year $(2 \%)$, or $8^{\text {th }}$ year $(1 \%$; APPIC, 2018$)$. Though all participants in the present study were enrolled in APA-accredited programs, the training programs the participants were enrolled in vary on many levels, such as direct service hour requirement, practicum eligibility and enrollment, and timeline of courses. For example, some programs may offer trainees the opportunity to begin training at a practicum site during their first or second semester, whereas other training sites may require a year of coursework prior to participation in clinical practicums. Perhaps future research can explore both training year and various components 
within training (e.g., how many semesters of practicum have been taken, current enrollment at an internship site, etc.) that can help to specify and account for aspects of training that may have indirectly influenced the outcomes in the present study.

\section{Conclusion}

The general finding of this study illustrates the relationship that mindfulness and selfcompassion have with counselor self-efficacy, self-assessed professional competency and training year. Thus, the thorough discussion on implementation of mindfulness and selfcompassion practices in educational training and applied practice settings is essential to not only initiate a movement toward intentionally including these practices throughout training but also to direct future research to identify more effective ways to implement these practices. As researchers further explore evidence-based practices that may be effective with a trainee population, instructors and supervisors should consider various aspects of mindfulness, selfcompassion, counselor self-efficacy and perceived competency as they creatively restructure their training programming.

Based on our findings, it would be beneficial for supervisors and educators to consider ways to teach and train mindfulness and self-compassion practices to their trainees. In order to do this, instructors and supervisors must first gain training and experience in these areas themselves. As the literature on self-compassion and mindfulness continues to grow, the ways in which these practices are taught constantly changes. Thus, just as with most domains of professional psychology, it is important to consider mindfulness and self-compassion, as well as counselor self-efficacy and perceived competency, as lifelong learning processes. Lifelong learning includes both mindset and action components where individuals actively participate in activities to enhance their competence in specific domains (Taylor \& Neimeyer, 2015). 
Therefore, it is incorrect to think that counselor self-efficacy and perceived competency, let alone mindfulness and self-compassion, could be achieved over the course of graduate training. Instead, these variables should be perceived through the lens of lifelong learning. The lifelong learning process can be modeled to trainees through the practices of their instructors and supervisors.

Overall, with the findings of the present study in mind, it is imperative to find ways to implement mindfulness and self-compassion practices into doctoral training for psychology students. Not only are these practices associated with enhanced trainees' levels of counselor selfefficacy and perceived competency, but they appear to have significant impacts on individuals' general personal and professional development. By incorporating and integrating mindfulness and self-compassion into classroom curriculum and supervision, trainees may respond more openly to the material provided and be able to connect more fully with their roles as cliniciansin-training (e.g., Fulton, 2016; Gockel et al., 2013). 


\section{REFERENCES}

Al-Darmaki, F. (2004). Counselor training, anxiety, and counseling self-efficacy: Implications for training psychology students from the United Arab Emirates University. Social Behavior and Personality, 32(5), 429-440. doi: 10.2224/sbp.2004.32.5.429

American Psychological Association (APA). (n.d.). Search for accredited programs [webpage]. Retrieved from https://apps.apa.org/accredsearch/?_ga=2.97683996.2061273075. $1553370353-447016725.1553370353$

American Psychological Association (APA). (2011). Benchmarks evaluation systems [webpage]. Retrieved from http://www.apa.org/ed/graduate/benchmarks-evaluation-system.aspx American Psychological Association (APA). (2012). A practical guidebook for the competency benchmarks [PDF]. Retrieved from http://www.apa.org/ed/graduate/guidebenchmarks.pdf

American Psychological Association (APA). (2016, December). Summary report: Student demographics [PDF]. Retrieved from https://www.apa.org/education/grad/surveydata/2017-student-demographics.pdf

American Psychological Association (APA). (2018). About APA: Our work [webpage]. Retrieved from http://www.apa.org/about/

Andersson, L., King, R., \& Lalande, L. (2010). Dialogical mindfulness in supervision role-play. Counselling and Psychotherapy Research, 10(4), 287-294. doi:

$10.1080 / 14733141003599500$

Association of Psychology Postdoctoral and Internship Centers (APPIC). (n.d.). Directory [Webpage]. Retrieved from https://membership.appic.org/directory/search Association of Psychology Postdoctoral and Internship Centers (APPIC). (2011). Appendix A: 
Internship data summaries [PDF]. Retrieved from https://www.appic.org/Portals/0/downloads/APPICDirectoryStats1112.pdf

Association of Psychology Postdoctoral and Internship Centers (APPIC). (2018). 2018 APPIC match: Survey of internship applicants part 1: Summary of survey results [Webpage]. Retrieved from https://www.appic.org/Internships/Match/Match-Statistics/ApplicantSurvey-2018-Part-1

Baer, R. A., Lykins, E. L. B., \& Peters, J. R. (2012). Mindfulness and self-compassion as predictors of psychological wellbeing in long-term meditators and matched nonmeditators. The Journal of Positive Psychology, 7, 230-238. doi: $10.1080 / 17439760.2012 .674548$

Baer, R. A., Smith, G. T., Hopkins, J., Krietemeyer, J., \& Toney, L. (2006). Using self-report assessment methods to explore facets of mindfulness. Assessment, 13, 27-45. doi: $10.1177 / 1073191105283504$

Bandura, A. (1977). Self-efficacy: Toward a unifying theory of behavior change. Psychological Review, 84, 191-215. doi: 10.1037/0033-295X.84.2.191

Baron, R. M., \& Kenny, D. A. (1986). The moderator-mediator variable distinction in social psychological research: Conceptual, strategic and statistical considerations. Journal of Personality and Social Psychology, 51, 1173-1182. doi: 10.1037/0022-3514.51.6.1173

Baumeister, R. F., Heatherton, T. F., \& Tice, D. M. (1993). When ego threats lead to selfregulation failure: Negative consequences of high self-esteem. Journal of Personality and Social Psychology, 64(1), 141-156. doi: 10.1002/3514/93

Belar, C. D., Brown, R. A., Hersch, L. E., Hornyak, L. M., Rozensky, R. H., Sheridan, E. P., Brown, R. T., \& Reed, G. W. (2001). Self-assessment in clinical health psychology: A model for 
ethical expansion of practice. Professional Psychology: Research and Practice, 32(2), 135-141. doi: 10.1037/0735-7028.32.2.I35

Bergen-Cico, D., \& Cheon, S. (2014). The mediating effects of mindfulness and self-compassion on trait anxiety. Mindfulness, 5(5), 505-519. doi: 10.1007/s12671-013-0205-y

Beutler, L. E., Malik, M., Alimohamed, S., Harwood, T. M., Talebi, H., Nobel, S., \& Wong, E. (2004). Therapist variables. In M. J. Lambert (Ed.), Bergin and Garfield's handbook of psychotherapy and behavior change (5 ${ }^{\text {th }}$ Ed.; pp. 227-306). New York: John Wiley \& Son, Inc.

Birnie, K., Speca, M., \& Carlson, L. E. (2010). Exploring self-compassion and empathy in the context of Mindfulness-Based Stress Reduction (MBSR). Stress and Health, 26(5), 359371. doi: 10.1002/smi.1305

Bishop, S. R., Lau, M., Shapiro, S., Carlson, L. E., Anderson, N. D., Carmody, J., Zindel, V. S., Abbey, S., Speca, M., Velting, D., \& Devins, G. (2004). Mindfulness: A proposed operational definition. Clinical Psychology: Science and Practice, 11, 230-241. doi: 10.1093/clipsy/bph077

Bluth, K. \& Blanton, P. W. (2014). Mindfulness and self-compassion: Exploring pathways to adolescent emotional well-being. Journal of Child and Family Studies, 23, 1298-1309. doi: $10.1007 / \mathrm{s} 10826-013-9830-2$

Bong, M., \& Skaalvik, E. M. (2003). Academic self-concept and self-efficacy: How different are they really? Educational Psychology Review, 15(1), 1-40. doi: 10.1040-726X/03/0300$0001 / 0$

Bradley, S., Drapeau, M., \& DeStefano, J. (2012). The relationship between continuing education and perceived competence, professional support, and professional value among 
clinical psychologists. Journal of Continuing Education in the Health Professions, 32(1), 31-38. doi: 10.1002/chp. 21120

Brown, K. W., \& Ryan, R. M. (2003a). Mindful Attention Awareness Scale [Database record]. Retrieved from http://dx.doi.org/10.1037/t04259-000

Brown, K. W., \& Ryan, R. M. (2003b). The benefits of being present: Mindfulness and its role in psychological well-being. Journal of Personality and Social Psychology, 84(4), 822-848. doi: $10.1037 / 0022-3514.84 .4 .822$

Bruce, N. G., Manber, R., Shapiro, S. L., \& Constantino, M. J. (2010). Psychotherapist mindfulness and the psychotherapy process. Psychotherapy Theory, Research, Practice, \& Training, 47(1), 83-97. doi: 10.1037/a0018842

Buser, T. J., Buser, J. K., Peterson, C. H., \& Seraydarian, D. G. (2012). Influence of mindfulness practice on counseling skills development. The Journal of Counselor Preparation and Supervision, 4(1), 20-36. doi: 10.7729/41.0019

Cohen, J. (1988). Statistical power analysis for the behavioral sciences ( $2^{\text {nd }}$ Ed.). Hillsdale, NJ: Erlbaum Associates.

Constantine, M. G. (2002). The relationship between general counseling self-efficacy and selfperceived multicultural counseling competence in supervisees. The Clinical Supervisor, 20(2), 81-90. doi: 10.1300/J001v20n02_07

Constantine, M. G., \& Ladany, N. (2001). New visions for defining and assessing multicultural counseling competence. In J. G. Ponterotto, J. M. Casas, L. A. Suzuki, \& C. M. Alexander (Eds.), Handbook of multicultural counseling (2 ${ }^{\text {nd }}$. Ed; pp. 482-498). Thousand Oaks, CA: Sage.

Cook, C., Heath, F., \& Thompson, R. L. (2000). A meta-analysis of response rates in web- or 
internet-based surveys. Educational and Psychological Measurement, 60, 821-836. doi: $10.1177 / 00131640021970934$

Damon, W. (1995). Greater expectations: Overcoming the culture of indulgence in America's homes and schools. New York: Free Press.

Deci, E. L., \& Ryan, R. M. (1995). Human autonomy: The basis for true self-esteem. In M.H. Kernis (Ed.), Efficacy, agency, and self-esteem (pp. 31-49). New York: Plenum.

Elliott, R., Bohart, A. C., Watson, J. C., \& Greenberg, L. S. (2011). Empathy. In J. Norcross (Ed.), Psychotherapy relationships that work (2 ${ }^{\text {nd }}$ Ed.; pp. 132-152). New York: Oxford University Press.

Epstein, R. M., \& Hundert, E. M. (2002). Defining and assessing professional competence. Journal of the American Medical Association, 287, 226-235. doi: 10.1001/jama.287.2.226

Eriksen, K., \& McAuliffe, G. (2003). A measure of counselor competency. Counselor Education Supervision, 43, 120-133. doi: 10.1002/j.1556-6978.2003.tb01836.x

Farb, N. A. S., Anderson, A. K., Mayberg, H., Bean, J., McKeon, D., \& Segal, Z. V. (2010). Minding one's emotions: Mindfulness training alters the neural expression of sadness. Emotion, 10(1), 25-33. doi: 10.1037/a0017151

Faul, F., Erdfelder, E., Lang, A. G., \& Buchner, A. (2007). G*Power 3: A flexible statistical power analysis program for the social, behavioral, and biomedical sciences. Behavior Research Methods, 39(2), 175-191. doi: 10.3758/BF03193146

Fauth, J., \& Williams, E. N. (2005). The in-session self-awareness of therapist-trainees: Hindering or helpful? Journal of Counseling Psychology, 52, 443-447. doi: 10.1037/0022-0167.52.3.443 
Feather, N. T. (1994). Attitudes toward high achievers and reactions to their fall: Theory and research concerning tall poppies. In M. Zanna (Ed.), Advances in experimental social psychology (pp. 1-73). San Diego, CA: Academic Press

Feltman, R., Robinson, M. D., \& Ode, S. (2009). Mindfulness as a moderator of neuroticismoutcome relations: A self-regulation perspective. Journal of Research in Personality, 43, 953-961. doi: 10.106/j.jrp.2009.08.009

Finlay-Jones, A., Kane, R., \& Rees, C. (2016). Self-compassion online: A pilot study of an internet-based self-compassion cultivation program for psychology trainees. Journal of Clinical Psychology, 73, 797-816. doi: 10.1002/jclp.22375

Fouad, N., Grus, C. L., Hatcher, R. L., Kaslow, N. J., Hutchings, P. S., Madson, M. B., Collins, F. L., \& Crossman, R. E. (2009). Competency benchmarks: A model for understanding and measuring competence in professional psychology across training levels. Training and Education in Professional Psychology, 3(4), S5-S26. doi: 10.1037/a0015832

Fredrickson, B. L., Cohn, M. A., Coffey, K. A., Pek, J., \& Finkel, S. M. (2008). Open hearts build lives: Positive emotions, induced through loving-kindness meditation, build consequential personal resources. Journal of Personality and Social Psychology, 95(5), 1045-1062. doi: 10.1037/a0013262

Friedlander, M. L., Keller, K. E., Peca-Baker, T. A., \& Olk, M. E. (1986). Effects of role conflict on counselor-trainee's self-statements, anxiety level, and performance. Journal of Counseling Psychology, 33, 73-77. doi: 10.1037/0022-0167.33.1.73

Friedlander, M. L., \& Snyder, J. (1983). Trainees' expectations for the supervisory process: Testing a developmental model. Counselor Education and Supervision, 16, 107-116. doi: 10.1002/j.1556-6978.1983.tb01771.x 
Fulton, C. L. (2016). Mindfulness, self-compassion, and counselor characteristics and session variables. Journal of Mental Health Counseling, 38(4), 360-374. doi:

10.17744/mehc.38.4.06

Fulton, C. L., \& Cashwell, C. S. (2015). Mindfulness-based awareness and compassion: Predictors of counselor empathy and anxiety. Counselor Education and Supervision, 54, 122-133. doi: 10.1002/ceas.12009

Germer, C. K. (2009). The mindful path to self-compassion. New York: Guilford Press.

Gilbert, P. (2010). Compassion focused therapies: Distinctive features. New York, NY: Routledge/Taylor \& Francis Group.

Gilbert, P, \& Proctor, S. (2006). Compassionate mind training for people with high shame and self-criticism: Overview and pilot study of a group therapy approach. Clinical Psychology and Psychotherapy, 13, 353-379. doi: 10.1002/cpp.507

Gockel, A., Burton, D., James, S., \& Bryer, E. (2013). Introducing mindfulness as a self-care and clinical training strategy for beginning social work students. Mindfulness, 4, 343-353. doi: $10.1007 / \mathrm{s} 12671-012-0134-1$

Goldin, P. R., \& Gross, J. J. (2010). Effects of Mindfulness-Based Stress Reduction (MBSR) on emotion regulation in social anxiety disorder. Emotion, 10(1), 83-81. doi: $10.1037 / \mathrm{a} 0018441$

Goreczny, A. J., Hamilton, D., Lubinski, L., \& Pasquinelli, M. (2015). Exploration of counselor self-efficacy across academic training. The Clinical Supervisor, 34(1), 78-97. doi: $10.1080 / 07325223.2015 .1012916$

Greason, P. B., \& Cashwell, C. S. (2009). Mindfulness and counseling self-efficacy: The mediating role of attention and empathy. Counselor Education and Supervision, 49, 2-19. 
doi: 10.1002/j.1556-6978.2009.tb00083.x

Grepmair, L., Mitterlehner, F., Loew, T., Bachler, E., Rother, W., \& Nickel, M. (2007).

Promoting mindfulness in psychotherapists in training influences the treatment results of their patients: A randomized double-blind, controlled study. Psychotherapy and Psychosomatics, 76, 332-338. doi: 10.1159/000107560

Grossman, P. (2011). Defining mindfulness by how poorly I think I pay attention during everyday awareness and other intractable problems for psychology's (re)invention of mindfulness: Comment on Brown et al. (2011). Psychological Assessment, 23(4), 10341040. doi: $10.1037 / \mathrm{a} 0022713$

Hiebert, B., Uhlemann, M. R., Marshall, A., \& Lee, D. Y. (1998). The relationship between selftalk, anxiety, and counselling skill. Canadian Journal of Counselling, 32, 163-171.

Hoerger, M. (2010). Participant dropout as a function of survey length in internet-mediated university studies: Implications for study design and voluntary participate-on in psychological research. Cyberpsychology, Behavior, and Social Networking, 13(6), 697700. doi: 10.1089/cyber.2009.0445

Holcomb-McCoy, \& C.C., Myers, J.E. (1999). Multicultural competence and counselor training: A national survey. Journal of Counseling and Development, 77(3), 294-302. doi: 10.1002/j.1556-6676.1999.tb02452.x

Hughes, A., Galbraith, D., \& White, D. (2011). Perceived competence: A common core for selfefficacy and self-concept? Journal of Personality Assessment, 93(3), 278-289. doi: $10.1080 / 00223891.2011 .559390$

Irving, J. A., Dobkin, P. L., \& Park, J. (2009). Cultivating mindfulness in health care professionals: A review of empirical studies of mindfulness-based stress reduction 
(MBSR). Complementary Therapies in Clinical Practice, 15, 61-66. doi:

10.1016/j.ctcp.2009.01.002

Ivers, N. N., Johnson, D. A., Clarke, P. B., Newsome, D. W., \& Berry, R. A. (2016). The relationship between mindfulness and multicultural counseling competence. Journal of Counseling \& Development, 94(1), 72-82. doi: 10.1002/jcad.12063

Jain, S., Shapiro, S L., Swanick, S., Roesch, S. C., Mills, P. J., Bell, I., \& Schwartz G. E. R. (2007). A randomized controlled trial of mindfulness meditation versus relaxation training: Effects on distress, positive states of mind, rumination, and distraction. Annals of Behavioral Medicine, 33(1), 11-21. doi: 10.1207/s15324796abm3301_2

Johnson, E., \& Seem, S. R. (1989, August). Supervisory style and the development of selfefficacy in counseling training. Poster session presented at the annual meeting of the American Psychological Association, New Orleans, LA.

Judd, C. M., McClelland, G. H., \& Ryan, C. S. (2009). Data analysis: A model comparison approach (2 $2^{\text {nd }}$ Edition). New York, NY: Routledge

Kabat-Zinn, J. (1990). Full catastrophe living: Using the wisdom ofyour body and mind to face stress, pain, and illness. New York: Delta.

Kabat-Zinn, J. (2003). Mindfulness-based interventions in context: Past, present, and future. Clinical Psychology: Science and Practice, 10, 144-156. Doi: 10.1093/clipsy.bpg016

Kaslow, N. J., Borden, K. A., Collins, F. L., Forrest, L., Illfelder-Kaye, J., Nelson, P., Rallo, J. S., Vasquez, M. J. T., \& Willmuth, M. E. (2004). Competencies conference: Future directions in education and credentialing in professional psychology. Journal of Clinical Psychology, 60, 699-712. doi: 10.1002/jclp.20016

Kozina, K., Grabovari, N., De Stefano, J., \& Drapeau, M. (2010). Measuring changes in 
counselor self-efficacy: Further validation and implications for training and supervision. The Clinical Supervisor, 29(2), 117-127. doi: 10.1080/07325223.2010.517483

Lamb, D. H., Baker, J. M., Jennings, M. L., \& Yarris, E. (1982). Passages of an internship in professional psychology. Professional Psychology, 13(5), 661-669. doi: 10.1037/07357028.13.5.661

Langer, E. (1989). Mindfulness. Reading, MA: Addison-Wesley.

Larson, L. M. (1998). The social cognitive model of counselor training. The Counseling Psychologist, 26, 219-273. doi: 10.1177/0011000098262002

Larson, L. M., \& Daniels, J. A. (1998). Review of the counseling self-efficacy literature. The Counseling Psychology, 26, 179-218. doi: 10.1177/0011000098262001

Larson, L. M., Suzuki, L. A., Gillespie, K. N., Potenza, M. T., Bechtel, M. A., \& Toulouse, A. L. (1992). Development and validation of the Counseling Self-Estimate Inventory. Journal of Counseling Psychology, 39(1), 105-120. doi: 10.1037/0022-0167.39.1.105

Lent, R. W., Hackett, G., \& Brown, S. D. (1998). Extending social cognitive theory to counselor training: Problems and prospects. The Counseling Psychologist, 26, 295-306. doi: $10.1177 / 0011000098262005$

Lent, R. W., Hill, C. E., \& Hoffman, M. A. (2003). Development and validation of the Counselor Activity Self-Efficacy Scales. Journal of Counseling Psychology, 50(1), 97-108. doi: $10.1037 / 0022-0167.50-1-97$

Leppma, M. (2012). Loving-kindness meditation and counseling. Journal of Mental Health Counseling, 34(3), 197-205. doi: 10.17744/mehc.34.3.955g218326616282

Leppma, M., \& Young, M. (2016). Loving-kindness meditation and empathy: A wellness group intervention for counseling students. Journal of Counseling and Development, 94(3), 
297-305. doi: 10.1002/jcad.12086

Margolies, R., Wachtel, A. B., \& Schmelkin, L. P. (1986). Perceived self-efficacy in psychiatry. Journal of Psychiatric Education, 10, 120-128. doi:

May, S., \& O’Donovan, A. (2007). The advantages of the mindful therapist. Psychotherapy in Australia, 13(4), 46-53.

McAlarnen, M. M. (2015). Becoming a mindful sport psychology consultant: Defining, developing, and integrating mindfulness into practice (Doctoral Dissertation). Retrieved from ProQuest Dissertations \& Theses Global. (3702007).

McCollum, E. E., \& Gehart, D. R. (2010). Using mindfulness meditation to teach beginning therapists therapeutic presence: A qualitative study. Journal of Marital and Family Therapy, 36(3), 347-360. doi: 10.1111/j.1752-0606.2010.00214.x

Melchert, T. P., Hays, V. L., Wiljanen, L. M., \& Kolocek, A. K. (1996). Testing models of counselor development with a measure of counseling self-efficacy. Journal of Counseling and Development, 74, 640-644. doi: 10.1002/j.1556-6676.1996.tb02304.x

Morgan, W. D., \& Morgan, S. T. (2005). Cultivating attention and empathy. In C. K. Germer, R. D., Siegel, \& P. R. Fulton (Eds.), Mindfulness and psychotherapy (pp. 73-90). New York: Guilford.

National Science Foundation. (2016). Statistical profile of doctorate recipients in social sciences fields, by sex and field of study; 2014 [PDF]. Retrieved from https://www.nsf.gov/statistics/2016/nsf16300/data/tab60.pdf

Neff, K. D. (2003a). The development and validation of a scale to measure self-compassion. Self and Identity, 2, 223-250. doi: 10.1080/15298860309027 
Neff, K. D. (2003b). Self-compassion: An alternative conceptualization of a healthy attitude toward oneself. Self and Identity, 2, 85-102. doi: 10.1080/15298860309032

Neff, K. D., (2016). The Self-Compassion Scale is a valid and theoretically coherent measure of self-compassion. Mindfulness, 7, 264-274. doi: 10.1007/s12671-014-0479-3.

Neff, K. D., \& Dahm, K. A. (2015). Self-compassion: What it is, what it does, and how it relates to mindfulness. In B. Ostafin, M.D. Robinson, \& B.P. Meier (Eds.), Handbook of mindfulness and self-regulation (pp. 121-137). New York: Springer.

Neff, K. D., \& Germer, C. K. (2013). A pilot study and randomized controlled trial of the mindful self-compassion program. Journal of Clinical Psychology, 69(1), 28-44. doi: $10.1002 /$ jclp.21923

Neff, K. D., Hsieh, Y., \& Dejitterat, K. (2005). Self-compassion, achievement goals, and coping with academic failure. Self and Identity, 4, 263-287. doi: 10.1080/13576500444000317

Neff, K. D., \& Pommier, E. (2013). The relationship between self-compassion and other-focused concern among college undergraduates, community adults, and practicing mediators. Self and Identity, 12(2), 160-176. doi: 10.1080/15298868.2011.649546

Neff, K. D., Rude, S. S., \& Kirkpatrick, K. L. (2007). An examination of self-compassion in relation to positive psychological functioning and personality traits. Journal of Research in Personality, 41, 908-916. doi: 10.1016/j.jrp.2006.08.002

O’Brien, K. M., Heppner, M. J., Flores, L. Y., \& Bikos, L. H. (1997). The career counseling selfefficacy scale: Instrument development and training applications. Journal of Counseling Psychology, 44, 20-31. doi: 10.1002/0167/97

Orzech, K. M., Shapiro, S. L., Brown, K. W., \& McKay, M. (2009). Intensive mindfulness training-related changes in cognitive and emotional experience. The Journal of Positive 
Psychology, 4(3), 212-222. doi: 10.1080/17439760902819394

Palcan, M. (2011). The mindful therapist trainee: An exploratory study of the in-session internal experiences of beginning therapists (Doctoral dissertation). Retrieved from ProQuest. (10107772).

Peterson, R. L., McHolland, J., Bent, R. J., Davis-Russell, E., Edwall, G. E., Polite, K., Singer, D. L., \& Stricker, G. (Eds). (1992). The core curriculum in professional psychology. Washington, D.C.: American Psychological Association.

Raab, K. (2014). Mindfulness, self-compassion, and empathy among health care professionals: A review of the literature. Journal of Health Care Chaplaincy, 20(3), 95-108. doi: $10.1080 / 08854726.2014 .913876$

Raes, F. (2011). The effect of self-compassion on the development of depression symptoms in a non-clinical sample. Mindfulness, 2, 33-36. doi: 10.1007/s12671-011-0040-y

Raes, F., Pommier, E., Neff, K. D., \& Gucht, D. V. (2011). Construction and factorial validation of a short form of the Self-Compassion Scale. Clinical Psychology and Psychotherapy, 18, 250-255. doi: 10.1002/cpp.702

Ridley, C. R., Mollen, D., \& Kelly, S. M. (2011). Beyond microskills: Toward a model of counseling competence. The Counseling Psychologist, 39(6), 1-40. doi: $10.1177 / 0011000010378440$

Roberts, M. C., Borden, K. A., Christiansen, M. D., \& Lopez, S. J. (2005). Fostering a culture shift: Assessment of competence in the education and careers of professional psychologists. Professional Psychology: Research and Practice, 36, 355-361. doi: $10.1037 / 0735.7028 .36 .4 .355$

Robins, T. G., Roberts, R. M., \& Sarris, A. (2015). Burnout and engagement in health profession 
students: The relationships between study demands, study resources and personal resources. Australian Journal of Organisational Psychology, 8(e1), 1-13. doi: 10.1017/orp. 2014.7

Rodolfa, E. R., Bent, R. J., Eisman, E., Nelson, P. D., Rehm, L., \& Ritchie, P. (2005). A cube model for competency development: Implications for psychology educators and regulators. Professional Psychology: Research and Practice, 36, 347-354. doi: $10.1037 / 0735-7028.36 .4 .347$

Roemer, L., Orsillo, S. M., \& Salters-Pedneault, K. (2008). Efficacy of an acceptance-based behavior therapy for generalized anxiety disorder: Evaluation in a randomized controlled trial. Journal of Consulting and Clinical Psychology, 76(6), 1083-1089. doi: $10.1037 / \mathrm{a} 0012720$

Rogers, C. R. (1957). The necessary and sufficient conditions of therapeutic personality change. Journal of Consulting Psychology, 21(2), 95-103. doi: 10.1037/h0055357

Ruggiero, S., Rabaino, A., Richards, D., \& Martin, W. E. (2014). Professional development and personal adjustment predictors of students' counseling self-efficacy. Paper presented at the annual conference of the Rocky Mountain Psychological Association, Denver, CO.

Salzberg, S. (1995). Loving-kindness: The revolutionary art of happiness. Boston, MA: Shambhala

Seligman, M. E. (1995). The optimistic child. Boston: Houghton Mifflin.

Schafer, J. L. (1999). Multiple imputation: A primer. Statistical Methods in Medical Research, 8, 3-15. doi: 10.1177/096228029900800102

Shahar, B., Szsepsenwol, O., Zilcha-Mano, S., Haim, N., Zamir, O., Levi-Yeshuvi, S., \& LevitBinnun, N. (2014). A wait-list randomized controlled trial of loving-kindness meditation 
programme for self-criticism. Clinical Psychology and Psychotherapy, 22(4), 346-356. doi: $10.1002 /$ cpp.1893

Shapiro, S. (2009). The integration of mindfulness and psychology. Journal of Clinical Psychology, 65(6), 555-560. doi: 10.1002/jclp.20602

Shapiro, S. L., Astin, J. A., Bishop, S. R., \& Cordova, M. (2005). Mindfulness-based stress reduction for health care professionals: Results from a randomized trial. International Journal of Stress Management, 12(2), 164-176. doi: 10.1037/1072-5245.12.2.164

Shapiro, S. L., Carlson, L. E., Astin, J. A., \& Freedman, B. (2006). Mechanisms of mindfulness. Journal of Clinical Psychology, 62(3), 373-386. doi: 10.1002/jclp.20237

Shapiro, S. L., \& Schwartz, G. E. R. (2000). Intentional systemic mindfulness: An integrative model for self-regulation and health. Advances in Mind-Body Medicine, 16(2), 128-134. doi: $10.1054 / \mathrm{ambm} .1999 .0118$

Shapiro, S. L., Schwartz, G. E., \& Bonner, G. (1998). Effects of mindfulness-based stress reduction on medical and premedical students. Journal of Behavioral Medicine, 21(6), 581-599. doi: 0160-7715/98/1200-0581

Sipps, G. J., Sugden, G. J., \& Favier, C. M. (1988). Counselor training level and verbal response type: Their relationship to efficacy and outcome expectations. Journal of Counseling Psychology, 35, 297-401. doi: 10.1037/0022-0167.35.4.397

Stoops, T. L. (2005). Understanding mindfulness: Implications for instruction and learning (Doctoral Dissertation). Retrieved from ProQuest Dissertations \& Theses Global. (320178).

Taylor, J. M. (2013). The development of the professional competencies scale: An assessment of foundational, functional, and continuing competencies for psychologists (Doctoral 
Dissertation). Retrieved from UF Theses \& Dissertations. (1542126847)

Taylor, J. M. (2015). Professional Competencies Scale [Database record]. Retrieved from PsycTESTS. http://dx.doi.org/10.1037/t14505-000

Taylor, J. M., \& Neimeyer, G. J. (2015). The assessment of lifelong learning in psychologists. Professional Psychology: Research and Practice, 46(6), 385-390. doi: 10.1037/pro0000027

Unsworth, S. K. P. M. (2015). Counselor self-efficacy: Relationship with mindfulness and selfcompassion (Doctoral dissertation). Retrieved from ProQuest. (3724862).

Urbani, S., Smith, M. R., Maddux, C. D., Smaby, M. H., Torres-Rivera, E., \& Crews, J. (2002). Skills-based training and counseling self-efficacy. Counselor Education and Supervision, 42(2), 92-106. doi: 10.1002/j.1556-6978.2002.tb01802.x

Van Dam, M. T., Earleywine, M., \& Borders, A. (2010). Measuring mindfulness? An item response theory analysis of the Mindful Attention Awareness Scale. Personality and Individual Differences, 49, 805-510. doi: 10.1016/j.paid.2010.07.020

Van Dam, N. T., van Vugt, M. K., Vago, D. R., Schmalzl, L., Saron, C. D., Olendzki, A., Meissner, T., Lazar, S. W., Kerr, C. E., Gorchov, J., Fox, K. C. R., Field, B. A., Britton, W. B., Brefczynski-Lewis, J. A., \& Meyer, D. E. (2018). Mind the hype: A critical evaluation and prescriptive agenda for research on mindfulness and meditation. Perspectives on Psychological Science, 13(1), 36-61. doi: 10.1177/1745691617709589

Walach, H., Buchheld, N., Buttenmuller, V., Kleinknecht, N., \& Schmidt, S. (2006). Measuring mindfulness: The Freiburg Mindfulness Inventory (FMI). Personality and Individual Differences, 40, 1543-1555. doi: 10.1016/j.paid.2005.11.025

Williams, E. N. Judge, A. B., Hill, C. E., \& Hoffman, M. A. (1997). Experiences of novice 
therapists in prepracticum: Trainees', clients', and supervisors' perceptions of therapists' personal reactions and management strategies. Journal of Counseling Psychology, 44, 390-399.

Ying, Y. (2009). Contribution of self-compassion to competence in mental health in social work students. Journal of Social Work Education, 45(2), 309-323. doi:

10.5175/JSWE.2009.200700072 


\section{APPENDICES}

\section{Appendix A: Informed Consent}

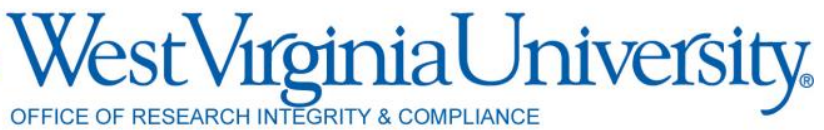

OFFICE OF RESEARCH INTEGRITY \& COMPLIANCE
Human Research Protocol Only Minimal Risk Consent

Form

Without HIPAA
Principal Investigator

Department

Psychology

Protocol Number

Study Title

Monica Leppma, Ph.D.

Counseling, Rehabilitation Counseling, and Counseling

1809291414

The Importance of Mindfulness and Self-Compassion in Clinical

Training: Outcomes Related to Self-Assessed Competency and Self-Efficacy in Psychologists-

in-Training

Co-Investigator(s)

Sponsor (if any)

Chelsea Latorre, M.Ed.

N/A

\section{Contact Persons}

If you have any questions, concerns, or complaints about this research, you can contact Chelsea Latorre via email at chlatorre@mix.wvu.edu

For information regarding your rights as a research subject, to discuss problems, concerns, or suggestions related to the research, to obtain information or offer input about the research, contact the Office of Research Integrity and Compliance at (304) 293-7073.

In addition if you would like to discuss problems, concerns, have suggestions related to research, or would like to offer input about the research, contact the Office of Research Integrity and Compliance at 304-293-7073.

\section{Introduction}

You have been asked to participate in this research study. This study is being conducted by Chelsea Latorre under the supervision of Dr. Monica Leppma in the Department of Counseling, Rehabilitation Counseling, and Counseling Psychology at West Virginia University with funding provided by the College of Education and Human Services.

\section{Purpose(s) of the Study}

The purpose of this study is to explore the development of professional/clinical experiences during graduate school and variables related to professional development and clinical work.

\section{Description of Procedures}

This study involves participation in an online survey that will take approximately 10 to 15 minutes for you to complete. You will be asked to fill out a questionnaire regarding your demographic information, your experiences within your training program, and your perceptions of your training qualifications. You do not have to answer all the questions.

\section{Discomforts}

There are no known or expected risks from participating in this study, except for the mild frustration associated with answering the questions.

\section{Benefits}

You may not receive any direct benefit from this study. The knowledge gained from this study may eventually benefit others.

\section{Financial Considerations}


You will be issued a $\$ 5$ gift card as a thank you for your participation. To receive the gift card, include your email address on the last page of the survey. Please note, for your privacy, email addresses are not linked with survey results.

\section{Confidentiality}

Any information about you that is obtained as a result of your participation in this research will be kept as confidential as legally possible. Your research records and test results, just like hospital records, may be subpoenaed by court order or may be inspected by the study sponsor or federal regulatory authorities (including the FDA if applicable) without your additional consent. Voluntary Participation

Participation in this study is voluntary. You are free to withdraw your consent to participate in this study at any time.

Refusal to participate or withdrawal will involve no penalty to you.

In the event new information becomes available that may affect your willingness to participate in this study, this information will be given to you so that you can make an informed decision about whether or not to continue your participation.

You have been given the opportunity to ask questions about the research, and you have received answers concerning areas you did not understand.

I willingly agree to be in the study.

$\circ$ Yes $\circ$ No 


\section{Appendix B: Participant Invitation Request}

Hello Dr. (training director/clinical director name),

My name is Chelsea Latorre and I am a doctoral candidate at West Virginia University. I am currently collecting data for my dissertation, which examines variables that influence competency for psychologists-in-training. I have attached the IRB approval (IRB \#: 1809291414), informed consent, eligibility requirements, and survey link below. Would you mind sending this out to students in your department that meet the eligibility criteria? Please contact me with any questions or concerns.

I appreciate your time!

Chelsea Latorre, M.Ed.

West Virginia University

Dear doctoral student,

This letter is a request for you to take part in a research project to explore the development of professional/clinical experiences during graduate school and variables related to professional development and clinical work. This project is being conducted by Chelsea Latorre, M.Ed., a doctoral candidate in counseling psychology, under the supervision of Dr. Monica Leppma at WVU. Chelsea is seeking help with data collection for her doctoral dissertation. Your participation in this project is greatly appreciated and will take approximately 10-15 minutes to fill out the attached questionnaire.

You will be issued a \$5 gift card as a thank you for your participation! To receive the gift card, just include your email address on the last page of the survey. Please note, for your privacy, email address are not linked with survey results.

Your involvement in this project will be kept as confidential as legally possible. All data will be reported in the aggregate. You must be 18 years of age or older; currently enrolled in an APA-accredited doctoral-level graduate program in clinical psychology, counseling psychology or combined counseling-clinical psychology to participate; and currently participating in a practicum/internship experience to participate. I will not ask any information that should lead back to your identity as a participant. Your participation is completely voluntary. You may discontinue your participation at any time and you may omit any question if you choose. West Virginia University's Institutional Review Board acknowledgement of this project is on file.

I hope that you will participate in this research project, as it could be beneficial in understanding relevant components of clinical training and professional development during graduate school. Thank you very much for your time. Should you have any questions about this letter or the research project, please feel free to contact Chelsea Latorre by e-mail at chlatorre@mail.wvu.edu or Dr. Monica Leppma by email at Monica.Leppma@mail.wvu.edu.

If you are willing to participate in this study, please click here:

https://wvu.qualtrics.com/jfe/form/SV_eYiegQUvNh3Wdet

Sincerely, Chelsea Latorre, M.Ed. \& Monica Leppma, Ph.D.

West Virginia University 


\section{Appendix C: IRB Approval}

\section{W. West VirginiaUniversity. \\ Office of Research Integrity and Compliance \\ 886 Chestnut Ridge Road Morgantown, WV 26506}

\section{Acknowledgement of Exemption}

$12 / 17 / 2018$

To: Monica Leppma

From: WVU Office of Research Integrity \& Compliance

Protocol Type: Exempt

Submission Type: Initial

Funding: N/A

WVU Protocol \#: 1809291414

Protocol Title: The Importance of Mindfulness and Self-Compassion in Clinical Training: Outcomes Related to Self-Assessed Competency and Self-Efficacy in Psychologists-in-Training
Approval Date: $12 / 17 / 2018$

Expiration Date: $12 / 16 / 2021$

The West Virginia University Institutional Review Board has reviewed your submission of Exempt protocol 1809291414. Additional details regarding the review are below:

- This research study was granted an exemption because the Research involves educational tests, survey procedures, interview procedures or observation of public behavior and (i) information obtained is recorded in such a manner that human subjects cannot be identified, directly or through identifiers linked to the subjects; and (ii) any disclosure of the human subjects responses outside the research could not reasonably place the subjects at risk of criminal or civil liability or be damaging to the subjects financial standing, employability, or reputation [45 CFR 46.101(2)]. All exemptions are only good for three years. If this research extends more than three years beyond the approved date, then the researcher will have to request another exemption. The following documents have been acknowledged for use in this study and are available in the WVU+kc system:

The following documents were reviewed and approved for use as part of this submission. Only the documents listed below may be used in the research. Please access and print the files in the Notes \& Attachments section of your approved protocol.

- Participant Invitation Request.pdf 
Referral List.docx

- Survey Items.pdf

- S0982318112010561.pdf

\section{WVU IRB acknowledgement of protocol 1809291414 will expire on 12/16/2021.}

If the study is to continue beyond the expiration date, a renewal application must be submitted no later than two (2) weeks prior to expiration date. It is your responsibility to submit your protocol for renewal.

Once you begin your human subjects research, the following regulations apply:

1. Unanticipated or serious adverse events and/or side effects encountered in this research study must be reported to the IRB within five (5) days, using the Notify IRB action in the electronic protocol.

2. Any modifications to the study protocol should be submitted only if there will be an increase in risk to subjects accompanying the proposed change(s).

3. You may not use a modified information sheet until it has been reviewed and acknowledged by the WVU IRB prior to implementation.

The Office of Research Integrity and Compliance will be glad to provide assistance to you throughout the research process. Please feel free to contact us by phone, at 304.293 .7073 or by email at IRB@mail.wvu.edu.

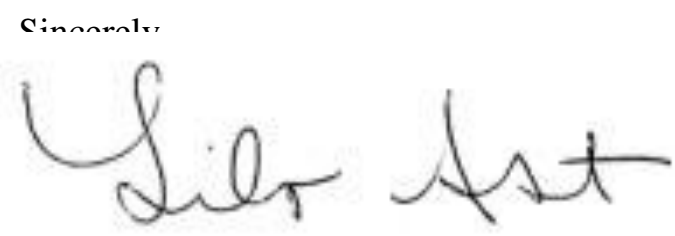

Lile Ast

IRB Administrator 


\section{Appendix D: Demographic Questionnaire}

1. What is your current age?

2. Which of the following describes your gender identity?

- Man

- Woman

- Transgender

- Gender nonconforming

- Other:

3. Which of the following best represents your ethnic/racial identity?

- African American or Black

- Asian American or Pacific Islander

- American Indian or Native American or Alaskan Native

- Arabic American or Middle Eastern

- Hispanic or Latinx

- East Indian or Indian American

- Caucasian American or White

- Biracial or Multiracial American

○ Please specify:

- Other:

4. What type of program are you currently enrolled in?

- Counseling Psychology

- Clinical Psychology

- Combined Clinical-Counseling Psychology

- Other:

5. What degree are you currently pursuing?

- Ph.D.

- Psy.D.

- Other:

6. What is your current year in your doctoral program?
- 1
- 2
- 3
- 4
- 5
- 6
- 7
- Other:

7. My current and primary practicum/internship site is...

- Community agency

- Consortium

- Correctional Facility

- Group Practice

- Hospital or Medical Setting 
- Independent Practice

- University Counseling Center

- VA Medical Center

- Other:

- I am not at a practicum/internship site currently

8. I engage in the following practices regularly (check all that apply):

- Meditation

- Yoga

- Martial Arts

- Qigong

- Relaxation exercises

- Mindful walking

- Mindful eating

- Self-Compassion breaks

- Journaling/Writing

- Other (please specify: 


\section{Appendix E: Mindful Attention Awareness Scale (MAAS)}

Below is a collection of statements about your everyday experience. Using the 1-6 scale below, please indicate how frequently or infrequently you currently have each experience. Please answer according to what really reflects your experience rather than what you think your experience should be.

$\begin{array}{cccccc}\text { Almost } & \text { Very } & \text { Somewhat } & \text { Somewhat } & \begin{array}{c}\text { Very } \\ \text { Infrequently }\end{array} & \begin{array}{c}\text { Almost } \\ \text { Never }\end{array} \\ \text { Always } & \text { Frequently } & \text { Frequently } & \text { Infrequently } & \text { Infrequent } \\ 1 & \mathbf{2} & \mathbf{3} & \mathbf{4} & \mathbf{5} & \mathbf{6}\end{array}$

1. I could be experiencing some emotion and not be conscious of it until some time later.

2. I break or spill things because of carelessness, not paying attention, or thinking of something else.

3. I find it difficult to stay focused on what's happening in the present.

4. I tend to walk quickly to get where I'm going without paying attention to what I experience along the way.

5. I tend not to notice feelings of physical tension or discomfort until they really grab my attention.

6. I forget a person's name almost as soon as I've been told it for the first time.

7. It seems I am "running on automatic" without much awareness of what I'm doing.

8. I rush through activities without being really attentive to them.

9. I get so focused on the goal I want to achieve that I lose touch with what I am doing right now to get there.

10. I do jobs or tasks automatically, without being aware of what I'm doing.

11. I find myself listening to someone with one ear, doing something else at the same time.

12. I drive places on "automatic pilot" and then wonder why I went there.

13. I find myself preoccupied with the future or the past.

14. I find myself doing things without paying attention.

15. I snack without being aware that I'm eating 


\section{Appendix F: Self-Compassion Scale Short-Form (SCS-SF)}

Please read each statement carefully before answering. Indicate how often you behave in the stated manner, using the following scale:

\section{Almost Never}

1
2
4

\section{Almost Always} 5

1. When I fail at something important to me I become consumed by feelings of inadequacy.

2. I try to be understanding and patient towards those aspects of my personality I don't like.

3. When something painful happens I try to take a balanced view of the situation.

4. When I'm feeling down, I tend to feel like most other people are probably happier than I am.

5. I try to see my failings as part of the human condition.

6. When I'm going through a very hard time, I give myself the caring and tenderness I need.

7. When something upsets me I try to keep my emotions in balance.

8. When I fail at something that's important to me, I tend to feel alone in my failure

9. When I'm feeling down I tend to obsess and fixate on everything that's wrong.

10. When I feel inadequate in some way, I try to remind myself that feelings of inadequacy are shared by most people.

11. I'm disapproving and judgmental about my own flaws and inadequacies.

12. I'm intolerant and impatient towards those aspects of my personality I don't like 


\section{Appendix G: Counselor Self-Efficacy Scale (CSES)}

Please read each statement carefully before answering. Indicate your agreement with each item using the following scale:

$\begin{array}{ccccc}\text { Agree } & \text { Agree } & \text { Neutral/ } & \text { Disagree } & \text { Disagree } \\ \text { Strongly } & \text { Moderately } & \text { Uncertain } & \text { Moderately } & \text { Strongly } \\ \mathbf{1} & \mathbf{2} & \mathbf{3} & \mathbf{4} & \mathbf{5}\end{array}$

1. My knowledge of personality development is adequate for counseling effectively.

2. My knowledge of ethical issues related to counseling is adequate for me to perform professionally.

3. My knowledge of behavior change principles is not adequate.

4. I am not able to perform psychological assessment to professional standards.

5. I am able to recognize the major psychiatric conditions.

6. My knowledge regarding crisis intervention is not adequate.

7. I am able to effectively develop therapeutic relationships with clients.

8. I can effectively facilitate client self-exploration.

9. I am not able to accurately identify client affect.

10. I cannot discriminate between meaningful and irrelevant client data.

11. I am not able to accurately identify my own emotional reactions to clients.

12. I am not able to conceptualize client cases to form clinical hypotheses.

13. I can effectively facilitate appropriate goal development with clients.

14. I am not able to apply behavior change skills effectively.

15. I am able to keep my personal issues from negatively affecting my counseling.

16. I am familiar with the advantages and disadvantages of group counseling as a form of intervention.

17. My knowledge of the principles of group dynamics is not adequate.

18. I am able to recognize the facilitative and debilitative behaviors of group members.

19. I am not familiar with the ethical and professional issues specific to group work.

20. I can function effectively as a group leader/facilitator. 


\section{Appendix H: Professional Competencies Scale- Revised (PCS-R)}

To what extent do you agree with each of the following in relation to your practice...

$\begin{array}{ccccc}\begin{array}{c}\text { Strongly } \\ \text { Disagree }\end{array} & \text { Disagree } & \begin{array}{c}\text { Neither Agree } \\ \text { Nor Disagree }\end{array} & \text { Agree } & \begin{array}{c}\text { Strongly } \\ \text { Agree }\end{array} \\ \mathbf{1} & \mathbf{2} & \mathbf{3} & 4 & 5\end{array}$

\section{Professionalism Scale}

1. I contribute to the development and advancement of the profession and colleagues.

2. I demonstrate integration of science in my professional practice.

Reflective Practice/Self-Assessment/Self-Care Scale

1. I accurately access my own strengths and weaknesses and seek to prevent or ameliorate the impact of any weaknesses on my professional functioning.

2. I recognize when new or improved competencies are required for effective practice.

Scientific Knowledge and Methods Scale

1. I routinely utilize scientific knowledge and skills in the solution of problems.

2. I readily present my own work for the scrutiny of others.

Relationships Scale

1. I effectively negotiate conflictual, difficult and complex relationships including those with individuals and groups that differ significantly from myself.

2. I accept, evaluate, and implement feedback from others.

Interdisciplinary Systems Scale

1. I demonstrate skill in interdisciplinary settings in order to incorporate psychological information into overall team planning and implementation.

2. I appreciate and integrate perspectives from multiple professions.

Assessment Scale

1. My interviews and reports lead to the formulation of appropriate diagnoses and treatment plans.

2. I interpret assessment results accurately, taking into account the limitations of the evaluation method.

\section{Intervention Scale}

1. I accurately assess presenting issues, taking into account the larger life context, including diversity issues.

2. I select and implement interventions appropriate for the presenting issue(s).

Consultation Scale

1. I demonstrate the ability to gather information necessary to answer referral questions.

2. I prepare clear, useful consultation reports and recommendations to all appropriate parties. 


\section{Appendix I: Figures Related to Statistical Assumptions}

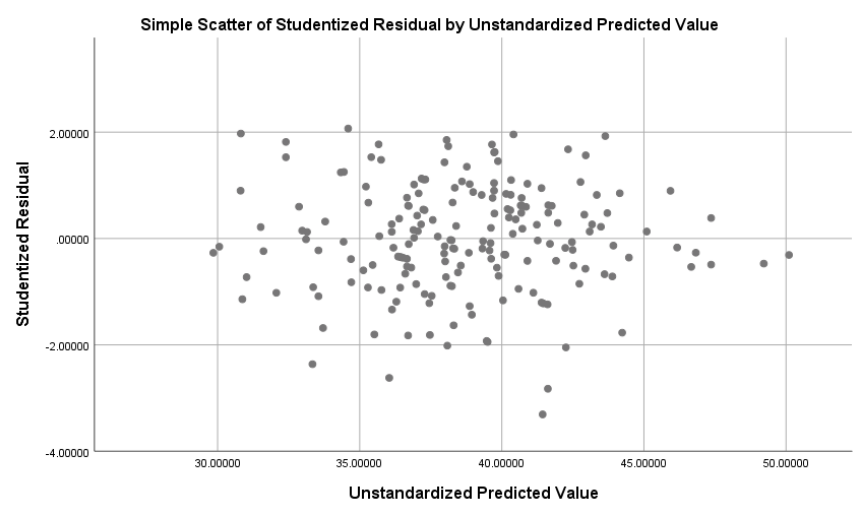

Figure 1. Scatterplot Testing Linearity and Homoscedasticity for Hypothesis One.

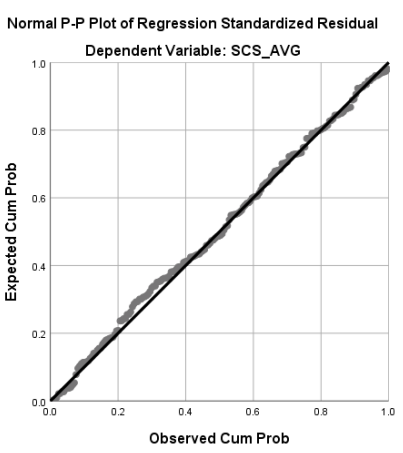

Figure 3. Normal P-P Plot Testing Normality for Hypothesis One.

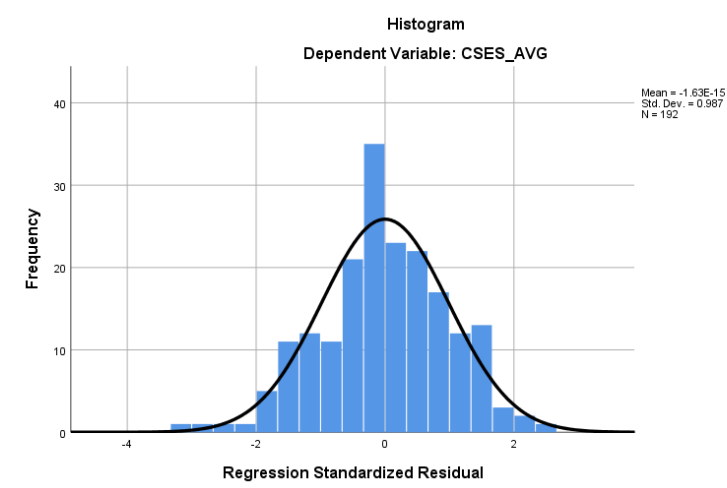

Figure 5. Histogram Testing Normality for Hypothesis Two and Three.

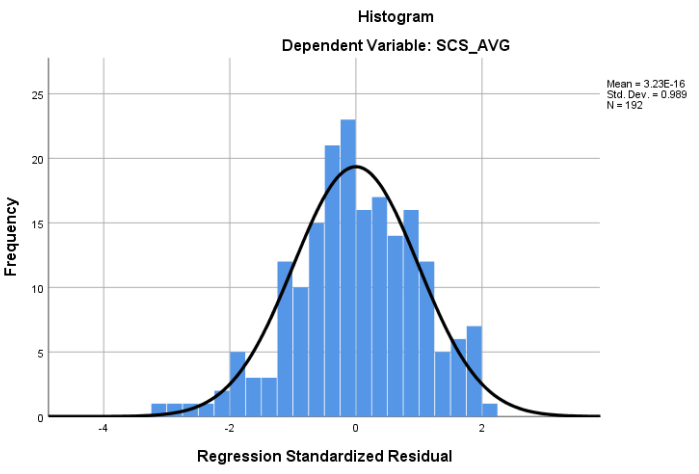

Figure 2. Histogram Testing Normality for Hypothesis One.

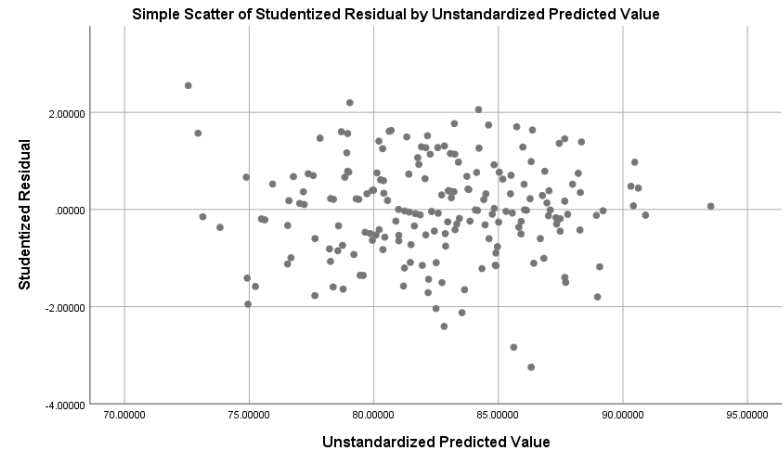

Figure 4. Scatterplot Testing Linearity and Homoscedasticity for Hypothesis Two and Three.

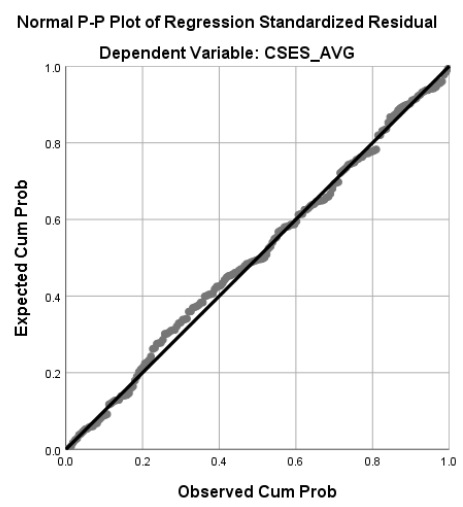

Figure 6. Normal P-P Plot Testing Normality for Hypotheses Two or Three. 


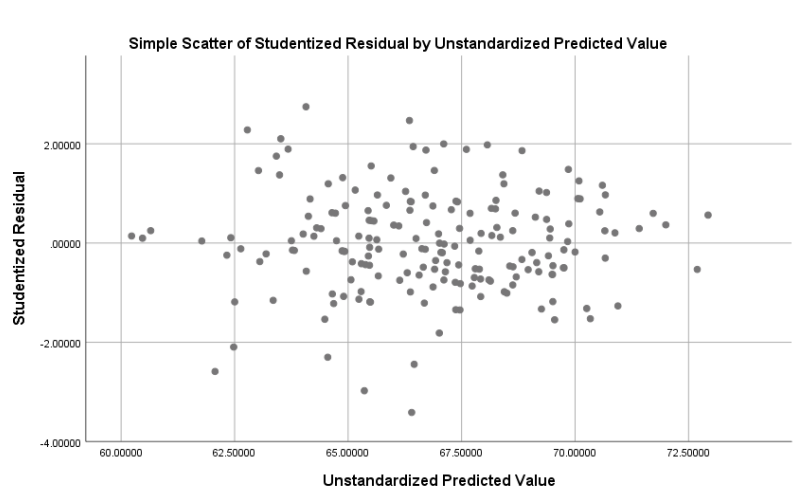

Figure 7. Scatterplot Testing Linearity and Homoscedasticity for Hypothesis Four and Five.

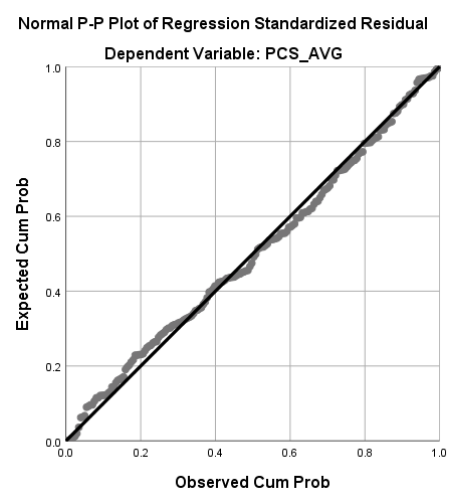

Figure 9. Normal P-P Plot Testing Normality for Hypothesis Four and Five.

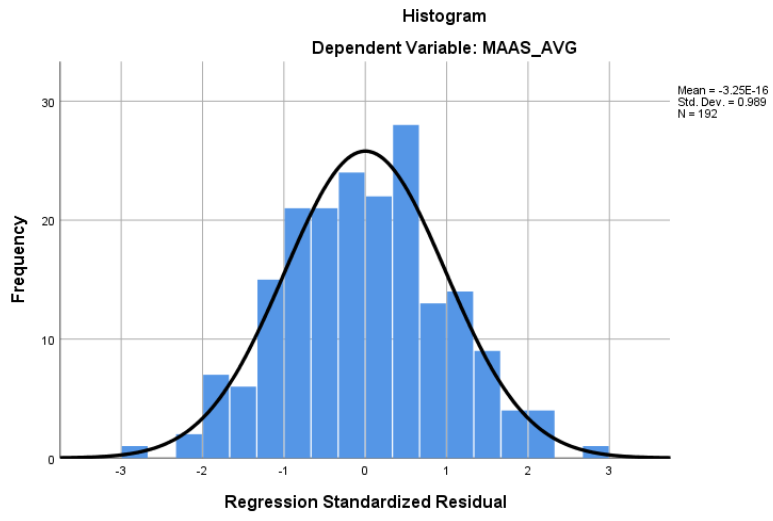

Figure 11. Histogram Testing Normality for Hypothesis Six.

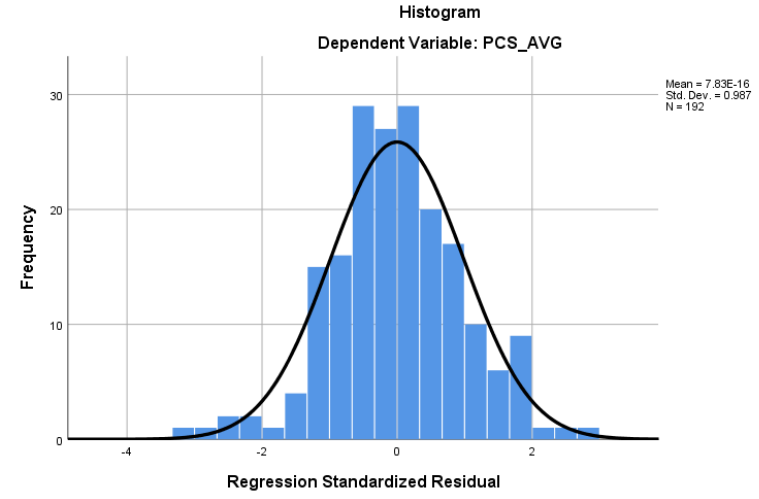

Figure 8. Histogram Testing Normality for Hypothesis Four and Five.

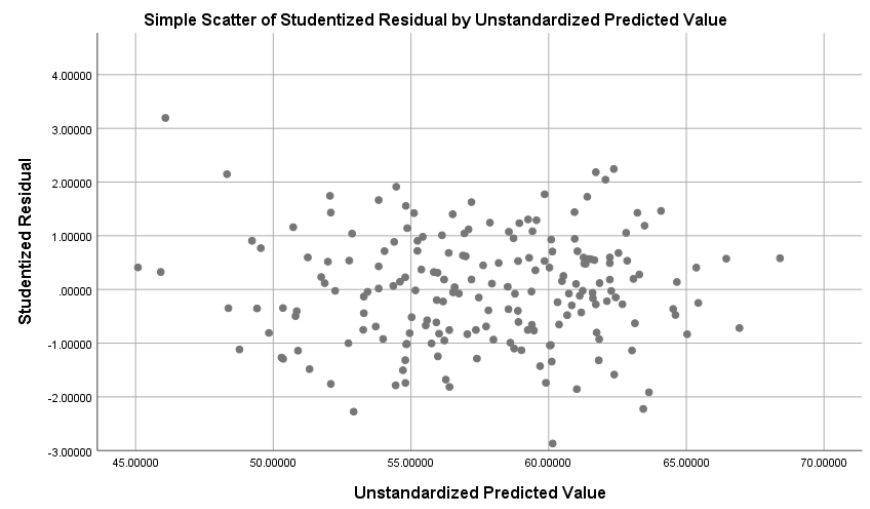

Figure 10. Scatterplot Testing Linearity and Homoscedasticity for Hypothesis Six.

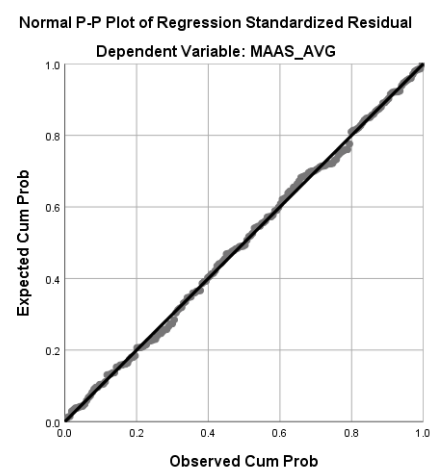

Figure 12. Normal P-P Plot Testing Normality for Hypothesis Six. 


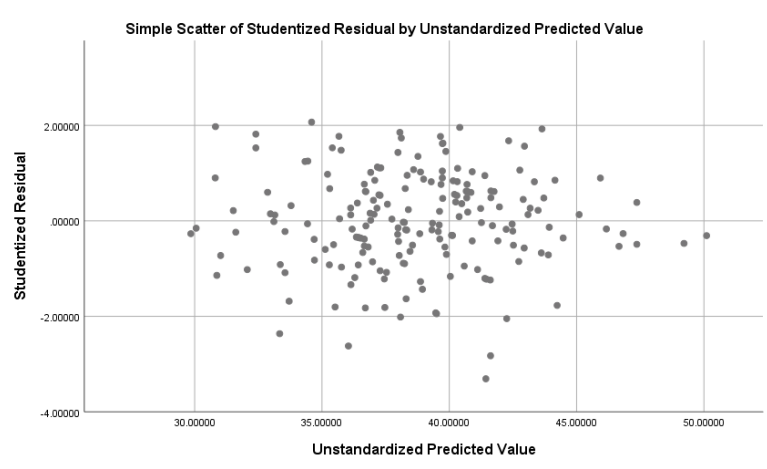

Figure 13. Scatterplot Testing Linearity and Homoscedasticity for Hypothesis Seven.

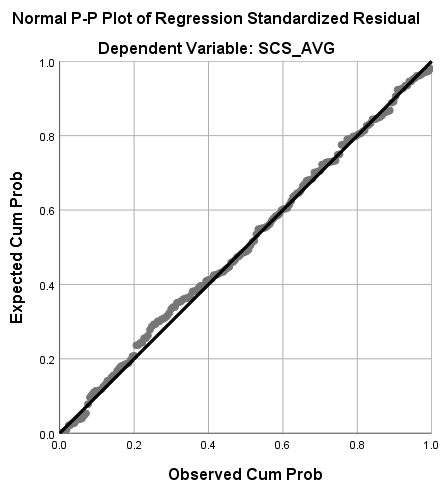

Figure 15. Normal P-P Plot Testing Normality for Hypothesis Seven.

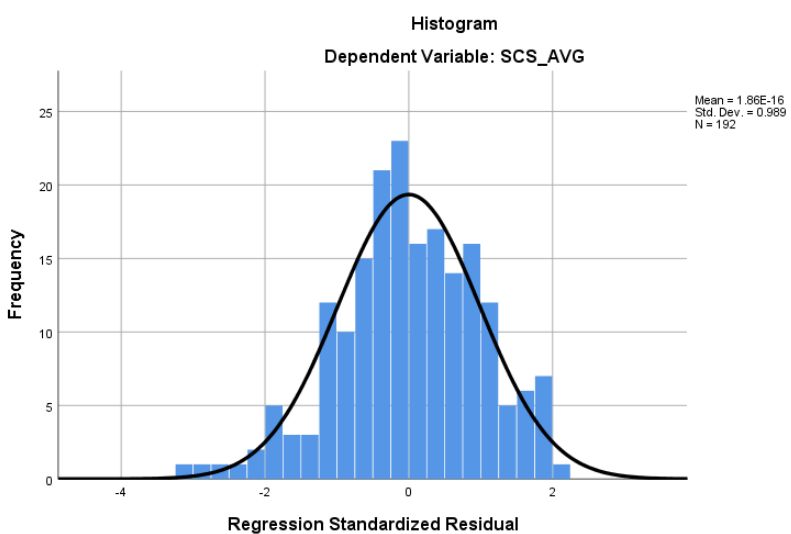

Figure 14. Histogram Testing Normality for Hypothesis Seven. 